\title{
Analyse des \\ Standardsetzungsprozesses von \\ IFRS 15 anhand von \\ Unternehmensstellungnahmen \\ und Standarddokumenten
}

\subsection{Durchführung der Analyse}

\subsubsection{Fragestellungen}

Für die Analyse des Einflusses von Unternehmen sowie dem IASB auf die Entwicklung von Rechnungslegungsvorschriften bedarf es einer Prozessbetrachtung, die Aufschluss über den Zusammenhang zwischen dem Entscheidungsprozess in Form der Begründbarkeit verschiedener Bilanzierungsalternativen und ihrer Umsetzungsmöglichkeiten - sowie dem finalen Standardergebnis ermöglicht. Im Gegensatz zu anderen Forschungsbeiträgen werden hierzu nicht kausale Zusammenhänge der Interessenabwägung, sondern der Standardsetzungsprozess als diskursive Auseinandersetzung untersucht. Im Sinne des konstruktivistischen Forschungsansatzes wird die Standardsetzung daher als ,wechselseitige[r] Konstitutionsprozess[.]" verstanden, in dem Regelungsvorschriften in ihrem Bedeutungsgehalt durch diskursive Akteure in einem diskursiven Kontext bestimmt werden. ${ }^{1}$ Diese „exercise in sense-making“ ${ }^{2}$ geht mit der Rechtfertigung einer Regelung in einem argumentativen bzw. sprachlichen Kontext einher. ${ }^{3}$ Die

\footnotetext{
${ }^{1}$ Vgl. Brand (2012), S. 217 (auch Zitat); zum Konstruktivismus in der Betriebswirtschaft Fülbier (2004), S. 269.

${ }^{2}$ Young (2003), S. 621.

${ }^{3}$ Vgl. Green (2004), S. 655.
}

Ergänzende Information Die elektronische Version dieses Kapitels enthält Zusatzmaterial, auf das über folgenden Link zugegriffen werden kann https://doi.org/10.1007/978-3-658-36129-7_3.

A. Jendreck, Die Rolle von Unternehmen im IFRS-Standardsetzungsprozess, Rechnungswesen und Unternehmensüberwachung, https://doi.org/10.1007/978-3-658-36129-7_3 
Rechtfertigung einer Regelungsvorschrift beruht auf dem (Vor-)Verständnis bzw. den allgemein verbreiteten Annahmen der am Prozess beteiligten Akteure, die zugleich genutzt werden, um die Überarbeitung bzw. Produktion neuer Regelungsvorschriften zu gestalten. ${ }^{4}$ Die Beziehung zwischen dem IASB sowie den Unternehmen und der Standardsetzung wird daher als konstitutiv ${ }^{5}$ betrachtet. Die von den Akteuren verfolgten Ziele und Interessen werden insofern als diskursiv konstituiert angesehen, als die Strukturen eines dominanten Diskurses die erfolgreiche Artikulation bestimmter Interessen ermöglichen. ${ }^{6}$ Unter der Annahme der Konkurrenz verschiedener Bedeutungsalternativen ist es daher „,von Interesse [...] zu eruieren, welche Bedeutungsgehalte wirkmächtig (i.S. von »mehrheitsfähig «, »akzeptiert«, weitestgehend erfolgreich diskursiv etabliert [...]) werden und welche konkurrierenden Bedeutungsgehalte durch diese Art der »Übereinkunft« tendenziell ausgegrenzt werden. “7 Stellungnahmen und Standarddokumente werden daher ,als Einsatz bzw. Ergebnis von diskursiven Praktiken [...] interpretiert" ${ }^{* 8}$.

Zunächst werden die im Standardsetzungsprozess kommunizierten Möglichkeiten der Standardausrichtung als inhaltliche Zielrichtung ${ }^{9}$ erfasst. Die im Rahmen der Standardsetzungsbestrebungen adressierten Bilanzierungsprobleme werden von den unterschiedlichen Auffassungen der am Prozess Beteiligten sowie der im Zuge des Prozesses als realisierbar erscheinenden Bilanzierungslösungen beeinflusst, ${ }^{10}$ weshalb die inhaltliche Zielrichtung die Bilanzierungsalternativen sowie deren Umsetzungsmöglichkeiten umfasst. Mithilfe der Regelungen des IFRS 15 zur Vertragszusammenfassung, zur Identifizierung und Aufteilung von Leistungsverpflichtungen sowie zur Aufteilung des Transaktionspreises wird sich dabei der Frage des Wandels eines Rechnungslegungssystems genähert, um Einblicke über das rekursive Verhältnis zwischen Standardanwendung und Standardsetzung zu erlangen. Die Analyse soll hierbei den ,discursive struggle"11 zwischen einer an konzeptionellen Idealen ausgerichteten Regelungsausgestaltung, die durch wesentliche Veränderungen bzw. eine Neuausrichtung

\footnotetext{
${ }^{4}$ Vgl. Green (2004), S. 655 f.

${ }^{5}$ Vgl. zur Unterscheidung kausaler und konstitutiver Beziehungen Wendt (1998), S. 104106.

${ }^{6}$ Vgl. Kurze (2018), S. 89-94.

${ }^{7}$ Vgl. Brand (2012), S. 218 (auch Zitat).

${ }^{8}$ Schneider/Janning (2006), S. 171.

${ }^{9}$ Vgl. zur Bezeichnung als inhaltliche Zielrichtung Tietz-Weber (2006), S. 107.

${ }^{10}$ Vgl. zur Problemdefinition in der Politikentwicklung Czada (1998), S. 58 f.

${ }^{11}$ Livesey (2001), S. 62; vgl. auch Higgins/Walker (2012), S. 196.
} 
der Regelungsvorschriften gekennzeichnet ist, und einer an der praktischen Anwendbarkeit anknüpfenden Regelungsausgestaltung, die durch die Beibehaltung bzw. nur graduelle Veränderung bestehender Regelungen zu charakterisieren ist, offenlegen und dadurch Rückschlüsse auf das Standardsetzungsergebnis ermöglichen. ${ }^{12}$ Der Standardsetzungsprozess des IFRS 15 zu den Regelungen der Vertragszusammenfassung sowie zur Aufteilung von Leistungsverpflichtungen und zur Aufteilung des Transaktionspreises stellt aufgrund seiner Konflikthaftigkeit $^{13}$ eine geeignete Untersuchungsbasis dar, da die Entwicklung der Regelungen konzeptionell von der Schließung der unter IAS 18 bestehenden Regelungslücke für Mehrkomponentengeschäfte bzw. der Reduzierung der nach US-GAAP bestehenden zahlreichen Einzelvorschriften angetrieben wurde, ${ }^{14}$ gleichzeitig jedoch aufgrund der von Unternehmen etablierten Bilanzierungspraxis sowie der hohen Praxisrelevanz ein in der Praxis anwendbarer Lösungsansatz angestrebt wurde.

Der Standardsetzungsprozess dient unter dem Legitimationsgesichtspunkt der Kommunikation der Gültigkeit angebrachter Regelungsvorschläge, wobei die Überzeugungskraft von der Argumentation sowie anderen sprachlichen Aspekten, mithin von der Rhetorik, abhängt. ${ }^{15}$ Durch die (strategische) Verwendung von Argumenten und rhetorischen Stilmitteln kann ,,[e]in bestimmtes Verständnis von Begriffen (und damit einhergehend eine bestimmte Auffassung über die und Ausgestaltung der [Regelungen])“ etabliert werden. ${ }^{16}$ Die Einflussnahme der am Standardsetzungsprozess Beteiligten wird dabei anhand des diskursiven Erfolgs beurteilt, der als ,Definitionsmacht und Deutungsmacht in Form der erfolgreichen Etablierung und Interpretation bestimmten Vokabulars und damit: der Prägung von Bedeutungsgehalten“"17 verstanden wird. Im Gegensatz zu bestehenden Lobbying-Studien wird somit keine empirische Überprüfung eines kausalen Zusammenhangs zwischen vertretenen Positionen und etwaigen Änderungen der Regelungsvorschriften vorgenommen, ${ }^{18}$ sondern der Entwicklungsprozess der Regelungen als diskursive Auseinandersetzung unter Berücksichtigung der Rolle von Rhetorik rekonstruiert. ${ }^{19}$ Als Untersuchungsgegenstand dient insofern nicht

${ }^{12}$ Vgl. Young (2003), S. $631 \mathrm{f}$.

${ }^{13}$ Vgl. zur Voraussetzung der Konflikthaftigkeit Schwab-Trapp (2011), S. 285 f.

${ }^{14} \mathrm{Vgl}$. IFRS 15.IN4.

15 Vgl. Fogarty (1994), S. 37 f.; Young (2003), S. 623 f.; Stenka (2013), S. 7 f.

${ }^{16}$ Vgl. zum Zusammenhang der Bedeutungsschaffung und des Sprachgebrauchs Baumann (2006), S. 78-84 (auch Zitat, S. 80).

${ }^{17}$ Brand (2012), S. 258, unter Zitierung von Baumann (2006), S. 81.

${ }^{18}$ Vgl. etwa Kwok/Sharp (2005), S. 82-93; Giner/Arce (2012), S. 665-680.

${ }^{19}$ Vgl. zur Abgrenzung zu bisherigen Studien Stenka/Jaworska (2019), S. 5. 
nur die inhaltliche, sondern auch die rhetorische Dimension. Dies ermöglicht die Identifizierung von Handlungsfäden, sog. story-lines, die als Narrative Verbindungen zwischen beiden Ebenen herstellen, mithin verschiedene Dimensionen der diskursiven Auseinandersetzung verknüpfen. ${ }^{20}$

Für die Ermittlung der zur Rechtfertigung der Bilanzierungslösungen verwendeten Argumente wird sich ausgehend von der Annahme diskursiver Strukturen - und insoweit in Übereinstimmung mit der in Lobbying-Studien bestehenden Einteilung $^{21}$ - zunächst an ökonomischen und konzeptionellen Begründungen orientiert, um herauszufinden, inwieweit sich einzelne Argumente im jeweiligen Diskurs wiederfinden bzw. diesen dominieren und die einzelnen Regelungsvorschriften in den vorgebrachten Argumenten begründet liegen ${ }^{22}$. Da sich im Rahmen der Kriterienauseinandersetzung sowohl auf wirtschaftliche Konsequenzen als auch bilanztheoretische Annahmen bezogen werden kann, ermöglicht die systematische Erfassung der Argumente dabei Rückschlüsse auf das (Vor-) Verständnis der Bilanzierungsalternativen sowie Tendenzen der Kompromissbildung. Rhetorik umfasst neben der (rationalen) Argumentationsebene indes weitere Mittel der Überzeugung, die zur Diskursetablierung auf der Glaubwürdigkeit des Autors oder der emotionalen Identifizierung der Adressaten gründen. ${ }^{23}$ Die Stellungnahmen und die Standardsetzungsdokumente werden daher auch auf andere rhetorische Strukturen sowie die verwendeten rhetorischen Stilmittel hin untersucht. ${ }^{24}$

Basierend auf der Forschungsfrage, wie Regelungsvorschriften durch das diskursive Standardsetzungsverfahren konstituiert werden, und dem konstruktivistischen Forschungsansatz erfolgt die Analyse nicht anhand überprüfbarer Hypothesen, sondern durch die Forschungsfrage konkretisierende Fragestellungen, ${ }^{25}$

\footnotetext{
${ }^{20}$ Vgl. Hajer (1995), S. 52-72.

${ }^{21}$ Vgl. Tutticci u. a. (1994), S. 94 f.; Jupe (2000), S. 346 f.; Stenka/Taylor (2010), S. 118; Giner/Arce (2012), S. 663; Wüstemann (2018), S. 15 f.

${ }^{22}$ Vgl. Yen u. a. (2007), S. 54; zur Verwendung bestimmter Argumente durch das FASB im Rahmen der Standardbegründung Young (2003), S. 628-632.

${ }^{23}$ Vgl. Higgins/Walker (2012), S. 197 f.

${ }^{24}$ Vgl. zur Berücksichtigung der Rhetorik beim Standardsetzungsprozess Young (2013), S. 878-881; Stenka (2013), S. 13-16; Stenka (2014), S. 16-22; Stenka/Jaworska (2019), S. 5-14.

${ }^{25}$ Vgl. zur Verwendung von Fragestellungen anstelle von Hypothesen Kwok/Sharp (2005), S. 78 f.; Auste (2011), S. 117-119, 133-135, 157 f.; Holder u. a. (2013), S. 138 f.
} 
die sich auf die inhaltliche sowie rhetorische Dimension ${ }^{26}$ des Standardsetzungsprozesses beziehen. Für die Analyse ergeben sich hieraus die folgenden zwei zentralen Fragestellungen:

Fragestellung 1: Welche inhaltlichen Zielrichtungen haben sich bei der Entwicklung der Regelungsvorschriften zur Vertragszusammenfassung sowie zur Aufteilung von Leistungsverpflichtungen und zur Aufteilung des Transaktionspreises im Laufe des Standardsetzungsprozesses von IFRS 15 etabliert?

Fragestellung 2: Welche rhetorischen Strategien haben sich bei der Entwicklung der Regelungsvorschriften zur Vertragszusammenfassung sowie zur Aufteilung von Leistungsverpflichtungen und zur Aufteilung des Transaktionspreises im Laufe des Standardsetzungsprozesses von IFRS 15 etabliert?

\subsubsection{Untersuchungsgegenstand}

\subsubsection{Unternehmensstellungnahmen und Standardentwürfe als Datenbasis}

Von der als Grundgesamtheit möglichen Datenbasis, zu der etwa auch Umfragen $^{27}$, Staff Paper ${ }^{28}$ oder Audioaufzeichnungen ${ }^{29}$ von Board-Meetings gehören können, wurden die von Unternehmen abgegebenen Stellungnahmen sowie die vom IASB veröffentlichten Standardentwürfe zu IFRS 15 und der finale Standard mit den jeweils zugehörigen Basis for Conclusions ${ }^{30}$ für die Analyse verwendet. Dieser Fokus liegt in der Forschungsfrage begründet, da Stellungnahmen und Standardentwürfe den im Standardsetzungsverfahren institutionalisierten Diskurs abbilden. In den Stellungnahmen kommen zugrundeliegende Routinen und Auffassungen zum Ausdruck; sie ermöglichen dadurch Einblicke in ,established ways to reason and argue, which constitute patterns of the dominant discourse

\footnotetext{
${ }^{26}$ Vgl. zur Einteilung für Analysezwecke in eine inhaltliche und rhetorische Dimension La Torre u. a. (2020), S. 5.

27 Vgl. etwa insgesamt Georgiou (2004).

${ }^{28} \mathrm{Vgl}$. etwa insgesamt Baudot (2018).

${ }^{29} \mathrm{Vgl}$. etwa insgesamt Klein/Fülbier (2018), S. 9.

${ }^{30}$ Vgl. ebenso Bennett u. a. (2006), S. 196.
} 
in the regulatory field." 31 Stellungnahmen werden auf Basis des bestehenden Vorwissens sowie im Kontext der zugehörigen Standardentwürfe schriftlich verfasst und von den Standardsetzern auf der jeweiligen Website veröffentlicht, weshalb die Entwicklung der Regelungsvorschriften unter Verwendung einer objektiv verfügbaren Datenbasis untersucht werden kann. ${ }^{32}$ Da zudem davon auszugehen ist, dass sich Stellungnehmer auch im Rahmen anderer Partizipationsmöglichkeiten während des Standardsetzungsprozesses ähnlich äußern, eignen sich Stellungnahmen in Einklang mit bisherigen Lobbying-Studien als „good proxy“. 33

Gleichzeitig erfordern Stellungnahmen als verpflichtender Verfahrensbestandteil des Due Process eine Auseinandersetzung des IASB mit den jeweiligen Aussagen, was wiederum eine Bezugnahme in den Standardentwürfen bzw. den finalen Standards erwarten lässt. Die Standardtexte und Begründungen geben daher nicht nur Aufschluss über die Ansichten des IASB, sondern auch über den Einfluss der Stellungnehmer. ${ }^{34}$ Die Standardentwürfe und der finale Standard sowie die jeweils zugehörigen Basis for Conclusions sind auf der Website der Standardsetzer öffentlich verfügbar und stellen daher ebenfalls eine vom Forschungsinteresse unabhängige Datenbasis dar.

Während in bisherigen Lobbying-Studien häufig das Verhältnis zwischen Stellungnahmen und Standardtexten durch eine Einflussanalyse in Form der Quantifizierung des Erfolgs untersucht wurde, ${ }^{35}$ stößt die vorliegende Dissertation durch den konstruktivistischen Ansatz in eine in der Rechnungslegungsstandardsetzung bestehende methodische Forschungslücke vor. Indem sowohl Stellungnahmen als auch Standardentwürfe durch eine diskursanalytische Perspektive unter Verwendung gleicher Untersuchungskriterien analysiert werden, kann die für die Entwicklung der Regelungen als maßgeblich angesehene diskursive Auseinandersetzung nachvollzogen werden. Zudem ermöglicht die Analyse der Stellungnahmen und Standardentwürfe über den gesamten Zeitraum der Standardsetzung Veränderungen dieser im Laufe der Zeit festzustellen.

\footnotetext{
${ }^{31}$ Vgl. Stenka/Jaworska (2019), S. 4 f. (auch Zitat, S. 4).

${ }^{32} \mathrm{Vgl}$. Orens u. a. (2011), S. 216.

${ }^{33}$ Vgl. Georgiou (2004), S. 230 (auch Zitat); zur Verwendung von Stellungnahmen als Datenbasis Watts/Zimmerman (1978), S. 122; Tutticci u. a. (1994), S. 86 f.; Kwok/Sharp (2005), S. 81 f.; Stenka/Taylor (2010), S. 111; Orens u. a. (2011), S. 216; Jorissen u. a. (2012), S. 703; Giner/Arce (2012), S. 660 f.; Bamber/McMeeking (2016), S. 64; Wüstemann (2018), S. 22; Stenka/Jaworska (2019), S. 4 f.

${ }^{34}$ Vgl. Young (2003), S. 626-636.

${ }^{35}$ Vgl. etwa Giner/Arce (2012), S. 664 f.; Shields u. a. (2019), S. 12 f.
} 
Alle für die Analyse verwendeten Stellungnahmen, die zum Discussion Paper, Exposure Draft und Re-Exposure-Draft eingegangen sind, stammen von der Website des FASB; die Stellungnahmen zum Exposure Draft der Clarifications $^{36}$ stammen von der Website des IASB. ${ }^{37}$ Die Standardentwürfe sowie der finale Standard und die zugehörigen Basis for Conclusions wurden ebenfalls von der Website des IASB bezogen. ${ }^{38}$ Während des Standardsetzungsprozesses von IFRS 15 wurden insgesamt 1.633 Stellungnahmen abgegeben, ${ }^{39}$ von denen nur die von Unternehmen erstellten Stellungnahmen in die Analyse einbezogen wurden (Tabelle 3.1). Die Eingrenzung auf Unternehmensstellungnahmen ${ }^{40}$ dient dazu, das rekursive Verhältnis zwischen Standardanwendung - insbesondere der Bilanzierungspraxis - und Standardsetzung zu untersuchen. Unternehmen sind als Anwender der Regelungen von einer Überarbeitung bestehender bzw. der Entwicklung neuer Vorschriften unmittelbar betroffen, weshalb für sie besonders im Fall erhöhter Implementierungskosten und/oder negativer Auswirkungen auf das Unternehmensergebnis ein hoher Anreiz zur Anfertigung einer Stellungnahme besteht. ${ }^{41}$ Vor allem große Unternehmen haben - aufgrund des in der Regel vorhandenen Fachwissens $-{ }^{42}$ dabei erwartungsgemäß eine positive Kosten-Nutzen-Bilanz. ${ }^{43}$

\footnotetext{
${ }^{36}$ Nach erstmaliger Veröffentlichung des Standards haben das IASB und FASB jeweils getrennte Standardentwürfe zu nachträglichen Klarstellungen veröffentlicht. Aufgrund des Fokus der vorliegenden Arbeit auf die Standardsetzung des IASB wurden nur die zum Exposure Draft der Clarifications des IASB eingegangenen Stellungnahmen einbezogen.

${ }^{37}$ Stellungnahmen die unaufgefordert eingesendet wurden, sog. ,,unsolicited comment letters", wurden nicht in die Analyse einbezogen, da für sie keine formale Grundlage zur Berücksichtigung für das IASB besteht.

${ }^{38}$ Der Standardentwurf zum Effective Date wurde nicht einbezogen, da dieser nicht die in der Analyse inhaltlich untersuchten Regelungen thematisiert.

${ }^{39}$ Die Gesamtanzahl der Stellungnahmen ergibt sich aus der Anzahl der auf der Website des FASB bzw. IASB veröffentlichten Stellungnahmen. Etwaige Abweichungen zu der laut Comment Letter Summarys abgegebenen Anzahl, wie bspw. 226 zu 211 zum DP (vgl. etwa Technical Staff (2009a), Agenda Paper 14 A/119 A, Rz. 3), können sich etwa aufgrund der Zusammengehörigkeit einzelner nicht untersuchter Stellungnahmen ergeben, die zwar einzeln eingereicht, jedoch als einer Institution zugehörig von den Standardsetzern zusammengefasst wurden.

${ }^{40}$ Vgl. zum Fokus auf Unternehmen Kelly (1982), S. 185; Kelly (1985), S. 620 f.; Brown/Feroz (1992), S. 716 f.; Saemann (1995), S. 556 f.; Larson (1997), S. 177 f.; Orens u. a. (2011), S. 212 f.; Wüstemann (2018), S. 5.

${ }^{41}$ Vgl. Watts/Zimmerman (1978), S. 118-121; Elbannan/McKinley (2006), S. 609-611.

${ }^{42}$ Vgl. Ordelheide (1997), S. 244 f.

${ }^{43}$ Vgl. Watts/Zimmerman (1978), S. 118-121; Kenny/Larson (1993), S. 539 f.; Larson (1997), S. 189 f.
} 
Die Stellungnahmen von Wirtschaftsprüfern, nationalen Standardsetzern und Nutzern wurden folglich ausgeschlossen. Einzelpersonen wurden nur dann einbezogen, wenn sie im Namen des Unternehmens geantwortet haben und diesem somit direkt zuordenbar waren. ${ }^{44}$ Die eingegangenen Stellungnahmen von Unternehmen aus dem Finanzsektor, konkret von Investment- und Versicherungsgesellschaften sowie Banken, wurden für die Analyse ebenfalls nicht berücksichtigt, da Versicherungsverträge sowie Verträge über Finanzinstrumente im Laufe des Standardsetzungsprozesses vom Anwendungsbereich des IFRS 15 ausgeschlossen wurden. ${ }^{45}$ Stellungnahmen von Unternehmens- und Wirtschaftsverbänden wurden aufgrund des Fokus der Analyse auf der direkten Einflussnahme ebenfalls nicht einbezogen. ${ }^{46}$ Da die Stellungnahmen nicht dichotom - bspw. in absolute Zustimmung oder Ablehnung - eingeteilt, sondern die jeweiligen inhaltlichen Zielrichtungen untersucht werden, wurden mehrere $\mathrm{zu}$ einem Standardentwurf abgegebene Stellungnahmen eines Unternehmens ${ }^{47}$ zu einer Stellungnahme zusammengefasst. ${ }^{48}$ Der sich aus diesem Vorgehen ergebende und für die Analyse verwendete Datensatz enthält insgesamt 512 Stellungnahmen ${ }^{49}$ sowie sechs Standardsetzungsdokumente des IASB ${ }^{50}$.

\footnotetext{
44 Vgl. zum analogen Vorgehen Larson (1997), S. 182; Jorissen u. a. (2012), S. 703. Die zum ED von 246 Einzelpersonen unterzeichnete Gruppenantwort (von den Standardsetzern als Comment Letter 666-912 erfasst) wurde aufgrund der fehlenden Unternehmensidentifikation folglich nicht einbezogen.

${ }^{45}$ Vgl. zum analogen Vorgehen Lam (2016), S. 285.

${ }^{46} \mathrm{Vgl}$. zur Unterteilung in direkte und indirekte Möglichkeiten der Einflussnahme Georgiou (2004), S. 222; Georgiou (2010), S. 106.

${ }^{47}$ Hierbei handelt es sich sowohl um mehrere Stellungnahmen derselben Einzelperson, die durch einen Buchstabenzusatz gekennzeichnet sind, wie bspw. Comment Letter 109 und 109 A zum ED, als auch um mehrere Stellungnahmen unterschiedlicher Personen, die jedoch zum gleichen Unternehmen gehören und im Namen des Unternehmens geantwortet haben, wie bspw. Comment Letter 259 und 554 zum ED.

48 Vgl. zur Annahme, dass Standardsetzer Stellungnahmen nicht zählen, sondern ihren Inhalt berücksichtigen Brown (1982), S. 282-290; Giner/Arce (2012), S. 666.

${ }^{49}$ Vgl. zur Übersicht aller einbezogenen Unternehmen in den einzelnen Kommentierungsphasen Appendix, I.

${ }^{50}$ Die sechs Standardsetzungsdokumente setzen sich aus dem Standardentwurf zum DP und zum ED Clarifications, die jeweils auch die Basis for Conclusions beinhalten, sowie der Basis for Conclusions zum ED, Re-ED, IFRS 15 (erste Veröffentlichung) und IFRS 15 (finaler Standard) zusammen. Im finalen Standard wurden nur die durch Buchstabenzusatz gekennzeichneten Abschnitte in die Analyse einbezogen.
} 
Tabelle 3.1 Gesamtanzahl der Stellungnahmen sowie Anzahl und Anteil der in die Analyse einbezogenen Unternehmensstellungnahmen in der jeweiligen Kommentierungsphase

\begin{tabular}{l|l|l|l|l|l}
\hline & $\begin{array}{l}\text { Discussion } \\
\text { Paper }\end{array}$ & $\begin{array}{l}\text { Exposure } \\
\text { Draft }\end{array}$ & $\begin{array}{l}\text { Re-Exposure } \\
\text { Draft }\end{array}$ & Clarifications & Gesamt \\
\hline Gesamtbeteiligung & 226 & 974 & 359 & 74 & 1.633 \\
\hline Unternehmensbeteiligung & 65 & 314 & 116 & 17 & 512 \\
\hline Unternehmensanteil & $28,76 \%$ & $32,24 \%$ & $32,31 \%$ & $22,97 \%$ & $31,35 \%$ \\
\hline
\end{tabular}

\subsubsection{Regelungen zur Bilanzierung von Mehrkomponentengeschäften als Untersuchungsgegenstand}

Die Untersuchung des Einflusses des IASB und der Unternehmen wird auf die Regelungen zur Vertragszusammenfassung sowie zur Aufteilung von Leistungsverpflichtungen und zur Aufteilung des Transaktionspreises beschränkt, da diese das Ergebnis eines der durch das Standardsetzungsprojekt verfolgten Ziele der Entwicklung von branchenübergreifenden Regelungen zur Bilanzierung von Mehrkomponentengeschäften - ${ }^{51}$ und damit das Resultat des formalen Entscheidungsprozesses darstellen. Mehrkomponentengeschäfte waren als in der Praxis entwickelte Vertragskonglomerate und Leistungsbündel bis zur Veröffentlichung von IFRS 15 nicht abschließend geregelt bzw. wurden in den US-GAAP in umfangreichen Einzelvorschriften adressiert, die auch im Rahmen der IFRSAnwendung herangezogen wurden. ${ }^{52}$ Das Konvergenzprojekt des IASB und FASB war aufgrund der geplanten konzeptionellen Neuausrichtung der Regelungen sowie des angestrebten branchenübergreifenden Ansatzes und der damit einhergehenden Veränderungen der bestehenden Praxis ${ }^{53}$ mit zahlreichen Kontroversen verbunden. So erstreckte sich die Entwicklung und Überarbeitung der Regelungen über fast alle Standardentwurfsphasen sowie für die Vorschriften zur Aufteilung von Leistungsverpflichtungen zusätzlich über die Phase der nachträglichen Klarstellungen. ${ }^{54}$ Die zur Bilanzierung von Mehrkomponentengeschäften relevanten Regelungsvorschriften stellen daher einen geeigneten Untersuchungsgegenstand dar, um die Regelungsentwicklung zu analysieren.

\footnotetext{
${ }^{51} \mathrm{Vgl}$. IFRS 15.IN4.

52 Vgl. Pilhofer u. a. (2010), S. 78-88; Fürwentsches (2010), S. 2-5.

${ }^{53}$ Vgl. Nösberger (2008), S. 460 f.; Marton/Wagenhofer (2010), S. 3.

${ }^{54}$ Vgl. DP (2008), Questions 4-7 und 11-13; ED (2010), Questions 1-3 und 7; ED Clarifications (2015), Question 1.
} 


\subsubsection{3 Überblick untersuchter Regelungen zur Bilanzierung von Mehrkomponentengeschäften nach IFRS 15}

\subsection{Ausnahmen vom Grundsatz der Umsatzerfassung auf Einzelvertragsbasis}

\subsection{Zusammenfassung von Verträgen}

Die Regelungen des IFRS 15 basieren auf einem vertragsbasierten Ansatz, der für die Erlöserfassung das Vorliegen eines Kundenvertrags verlangt (IFRS 15.6). Ein Vertrag ist durch die rechtliche Durchsetzbarkeit vereinbarter Rechte und Pflichten definiert (IFRS 15.10), was die vom IASB und FASB verfolgte Neuausrichtung der Regelungen am Asset-Liability-Ansatz widerspiegelt und insoweit einer zum Rahmenkonzept konsistenten Ausgestaltung entspricht. ${ }^{55}$ Neben der anhand nationalen Zivilrechts $\mathrm{zu}$ bestimmenden Durchsetzbarkeit ${ }^{56}$ sind die wirtschaftliche Substanz des Vertrags sowie Delkredererisiken zu beurteilen (IFRS 15.9). Obwohl die bilanzielle Erfassung an den jeweils einzeln geschlossenen Vertrag anknüpft, sind zwei oder mehr zeitgleich oder in geringem Zeitabstand geschlossene Verträge mit einem Kunden oder ihm nahestehenden Personen zusammenzufassen und als ein Vertrag zu bilanzieren, sofern die Verträge einem einzigen wirtschaftlichen Zweck dienen, eine Preisinterdependenz zwischen den Verträgen besteht und/oder die vereinbarten Vertragskomponenten eine einzige Leistungsverpflichtung darstellen (IFRS 15.17). Während im Discussion Paper die Zusammengehörigkeit mehrerer Verträge zwar erwähnt, jedoch nicht konkretisiert wurde, ${ }^{57}$ war im Exposure Draft neben der Zusammenfassung zweier oder mehrere Verträge im Fall bestehender Preisabhängigkeiten zwischen den Gütern oder Dienstleistungen auch die im umgekehrten Fall der Preisunabhängigkeit erforderliche Segmentierung eines Vertrags in mehrere Einzelverträge vorgesehen ${ }^{58}$. Das im Exposure Draft für die Vertragszusammenfassung relevante Kriterium der Preisabhängigkeit wurde im überarbeiteten Exposure Draft durch die bis dahin als Indikator genannte Bedingung der Zeitnähe ersetzt und stellt nach IFRS 15 kein notwendiges, sondern lediglich hinreichendes Kriterium dar

\footnotetext{
${ }^{55}$ Vgl. Wüstemann/Wüstemann (2014), S. 930 f.; Wüstemann u. a. (2017a), Rz. 13 f.

56 Vgl. IFRS 15.BC32; Schulzke u. a. (2013), S. 528-573; Heintges u. a. (2015), S. 570-575; Wüstemann u. a. (2017a), Rz. 17.

${ }^{57} \mathrm{Vgl}$. DP (2008), 2.27.

$58 \mathrm{Vgl}$. ED (2010), 12-16.
} 
(IFRS 15.17). ${ }^{59}$ Eine Regelungsvorschrift zur Vertragssegmentierung besteht hingegen nicht; einzelne Bestandteile eines Vertrags sind vielmehr im Rahmen der Identifizierung von Leistungsverpflichtungen abzugrenzen. ${ }^{60}$

Das Erfordernis der Vertragszusammenfassung in IFRS 15.17 ist als Ausnahmeregelung zu charakterisieren. ${ }^{61}$ Die Anforderung des zeitnahen Vertragsabschlusses mit der gleichen oder dieser nahestehenden Vertragspartei fungiert dabei als Restriktion, weshalb die Ausgestaltung der Verträge als Paket mit einem einzigen kommerziellen Ziel oder das Bestehen von Preisabhängigkeiten für eine Zusammenfassung nicht ausreichen. ${ }^{62}$ So folgt auch eine Agenda-Entscheidung des IFRS Interpretations Committee einer individualvertraglichen Bilanzierung. ${ }^{63}$ In Frage stand das Bestehen eines durchsetzbaren Zahlungsanspruchs bei Kündigungsmöglichkeit eines Immobilienvertrags durch den ursprünglichen Vertragspartner bei gleichzeitiger Verpflichtung des Unternehmens im Kündigungsfall zum Weiterverkauf durch Vertragsabschluss mit einem Dritten. ${ }^{64}$ Die einheitliche Betrachtung der „ökonomischen Gesamtwirkung des Vertragskonstrukts“ wird hierbei durch die vom rechtlichen Vertragsschluss abhängigen verschiedenen Zeitpunkte und unterschiedlichen Vertragsparteien überlagert. ${ }^{65}$ Die Entscheidung erscheint vor dem Hintergrund des ausstehenden Vertragsabschlusses mit einem Dritten mit einer am Regelungswortlaut und den Erwägungsgründen des IASB orientierten Auslegung gerechtfertigt. ${ }^{66}$ Mit dem zur Darstellung der Unternehmensperformance bezweckten zeitraumbezogenen Kontrollübergang nach IFRS 15 ist sie indes nur bedingt in Einklang zu bringen, da der Erhalt der Gegenleistung vom ursprünglichen Kunden oder einem Dritten für das bilanzierende Unternehmen in wirtschaftlicher Sicht keinen Unterschied darstellt ${ }^{67}$. Inwieweit diese Agenda-Entscheidung trotz ihrer faktischen Verbindlichkeit auf ähnliche Sachverhalte übertragen werden kann, dürfte letztlich von den jeweiligen Vertragsausgestaltungen abhängig sein. So sollten etwa Rahmenwerkverträge, die Hauptleistungspflichten beinhalten, sowie zugehörige Produktionsverträge,

\footnotetext{
${ }^{59} \mathrm{Vgl}$. Re-ED (2011), 17.

${ }^{60}$ Vgl. Re-ED BC (2011), BC49.

${ }^{61}$ Vgl. Schild (2019), S. 12.

${ }^{62}$ Vgl. IFRS 15.BC75; Schild (2019), S. 12 f.

${ }^{63}$ Vgl. IFRS Interpretations Committee (2018), Agenda Paper 2E; Technical Staff (2017a), Agenda Paper 2B, Rz. 20-26, Schild (2019), S. 13.

${ }^{64}$ Vgl. Technical Staff (2017a), Agenda Paper 2B, Rz. 4.

${ }^{65}$ Vgl. Schild (2019), S. 12 f. (auch Zitat, S. 12).

${ }^{66}$ Vgl. Technical Staff (2017a), Agenda Paper 2B, Rz. 20-26.

${ }^{67}$ Vgl. Technical Staff (2017a), Agenda Paper 2B, Appendix B, S. 19 f.
} 
wie z. B. die in der Automobilzuliefererindustrie gängigen separaten Verträge in Form von Rahmenvereinbarungen über allgemeine Lieferkonditionen sowie zur Werkzeugproduktion einerseits und andererseits zur Serienteilfertigung, zusammengefasst werden, wenn sie in einem aus wirtschaftlicher Sicht zusammenhängenden Zeitraum abgeschlossen werden. ${ }^{68}$ Gleiches sollte auch im Fall eines auf mehrere Einzelverträge verteilten Werklieferungsauftrags gelten, sofern die im jeweiligen Einzelvertrag vereinbarten Leistungen einem Gesamtfunktionsrisiko unterliegen. ${ }^{69}$ Die Regelungen zur Vertragszusammenfassung dienen dabei der sachgerechten Aufteilung des Transaktionspreises sowie der zeitlichen Umsatzerfassung ${ }^{70}$ erfordern jedoch im Einzelfall eine Ermessensausübung im Rahmen der Standardanwendung.

\subsection{Portfolio-Option}

Der Grundsatz der einzelvertragsbasierten Bilanzierung wird ebenfalls durch die Portfolio-Option des IFRS 15.4 beschränkt, wonach Unternehmen die Regelungen auf ein Portfolio ähnlich ausgestalteter Verträge oder Leistungsverpflichtungen anwenden können, sofern hieraus im Vergleich zur Bilanzierung der einzelnen Kundenverträge keine wesentlichen Unterschiede resultieren. Im Gegensatz zur verpflichtenden Vertragszusammenfassung bei Erfüllung von IFRS 15.17 stellt die Ausübung dieses sog. praktischen Behelfs ein Wahlrecht dar. ${ }^{71}$ Die Festlegung des Portfolios liegt dabei im Ermessen des Unternehmens, ${ }^{72}$ das auf Basis von vernünftigen Schätzungen und Annahmen die Größe und Zusammensetzung eines Vertragsportfolios zu bestimmen hat (IFRS 15.4). Diese Form der unternehmensspezifischen Zusammenfassung wurde erst im überarbeiteten Exposure Draft eingeführt und stellt eine anwendungsorientierte Erleichterung dar. ${ }^{73}$ Obwohl die Regelung eine Vereinfachung der Anwendung bezweckt, führte sie bereits nach erstmaliger Veröffentlichung des IFRS 15 zu Anwendungsfragen in Bezug auf die Schätzung variabler Vergütungsbestandteile, die durch die TRG adressiert wurden. ${ }^{74}$ Demnach ist die Portfolio-Betrachtung von der Verwendung historischer Erfahrungswerte auf Basis ähnlicher Verträge zur Schätzung des Erwartungswerts zu unterscheiden, d. h. während bspw. unterschiedliche Kundenverträge über das

\footnotetext{
${ }^{68}$ Vgl. Schurbohm-Ebneth/Viemann (2015a), S. 182; Wüstemann u. a. (2017b), S. 1196 f.

${ }^{69}$ Vgl. Lüdenbach u. a. (2016), § 25, Rz. 43.

${ }^{70}$ Vgl. IFRS 15.BC71; Lüdenbach u. a. (2016), § 25, Rz. 41.

${ }^{71}$ Vgl. Wüstemann u. a. (2017a), Rz. 34 f.

72 Vgl. IFRS 15.BC69; Grote u. a. (2014), S. 413; Brücks u. a. (2017), S. 180 f.

${ }^{73}$ Vgl. Re-ED (2011), 6; IFRS 15.BC69 f.

74 Vgl. TRG (2015a), Agenda Paper 38, Rz. 1 f.; TRG (2015b), Agenda Paper 44, Rz. 24.
} 
gleiche Produkt mit geringfügig unterschiedlichen Rückgabequoten als Portfolio betrachtet und auf alle Verträge die gleiche durchschnittsgewichtete Rückgabequote angewendet werden kann, gilt die Schätzung der Retourenquote für einen Einzelvertrag auf Basis ähnlicher Verträge nicht unmittelbar für ein Portfolio an Verträgen. ${ }^{75}$ Mithilfe der Portfolio-Option können demzufolge eine Vielzahl vergleichbarer Verträge gemeinsam beurteilt werden, ${ }^{76}$ was vor allem für Verträge „mit ähnlichen wirtschaftlichen Charakteristika“, wie bspw. vergleichbaren Vertragskonditionen, in Frage kommt; gleichzeitig sind aufgrund der Ausgestaltung als Wahlrecht sowie den bei dessen Ausübung bestehenden Ermessensspielräumen unternehmens- bzw. branchenspezifische Unterschiede in der Anwendung möglich. ${ }^{77}$

\subsection{Identifizierung von Leistungsverpflichtungen}

\subsection{Definition von Leistungsverpflichtungen}

Sofern ein (zusammengefasster) Kundenvertrag im Sinne von IFRS 15 vorliegt, hat ein Unternehmen bei Vertragsabschluss bestehende Leistungsverpflichtungen zu identifizieren (IFRS 15.22). Bei Leistungsverpflichtungen handelt es sich gemäß IFRS 15.22 um Leistungszusagen, eigenständig abgrenzbare Güter oder Dienstleistungen bzw. Leistungsbündel an den Kunden zu übertragen; auch eine Reihe eigenständig abgrenzbarer Güter oder Dienstleistungen, die als gleiche Leistungszusagen jeweils zeitraumbezogen in gleicher Weise auf den Kunden übertragen werden, wie etwa bei Reinigungs- oder Energielieferverträgen ${ }^{78}$, sind als einzelne Leistungsverpflichtung zu bilanzieren (IFRS 15.22 f.; IFRS 15 Anhang A). ${ }^{79}$ Während Leistungsverpflichtungen im Discussion Paper noch allgemein als Leistungszusagen zur Übertragung von Vermögenswerten definiert waren ${ }^{80}$ erfolgte ab dem Exposure Draft durch Bezugnahme zur eigenständigen Abgrenzbarkeit eine Konkretisierung der zu identifizierenden Leistungsverpflichtungen $^{81}$.

\footnotetext{
75 Vgl. TRG (2015a), Agenda Paper 38, Rz. 9-12; TRG (2015b), Agenda Paper 44, Rz. 25; kritisch zur Unterscheidung der TRG am Beispiel insignifikanter Retourenquoten Lüdenbach u. a. (2016), § 25, Rz. 183.

$76 \mathrm{Vgl}$. IFRS 15.BC70.

${ }^{77}$ Vgl. Brücks u. a. (2017), S. 180 f. (auch Zitat, S. 180).

78 Vgl. Pollmann u. a. (2017), S. 212.

${ }^{79} \mathrm{Vgl}$. IFRS 15.BC84; IFRS 15.BC113 f.

${ }^{80} \mathrm{Vgl}$. DP (2008), 3.2.

${ }^{81}$ Vgl. ED (2010), 20; Re-ED (2011), 23 f.
} 
Neben vertraglich festgelegten Leistungszusagen sind auch faktische Leistungszusagen, die aufgrund gerechtfertigter Kundenerwartungen einen wirtschaftlichen Leistungszwang begründen, zu berücksichtigen (IFRS 15.24). Mögliche Leistungszusagen sind gemäß IFRS 15.26 bspw. die (kundenspezifische) Herstellung und/oder der Verkauf von Waren sowie die Erbringung von Dienstleistungen wie auch die laufende Bereitstellung bzw. Bereithaltung von Gütern oder Dienstleistungen sowie die Gewährung von Lizenzen und Optionen. Von Unternehmen ausgeführte Erfüllungstätigkeiten, durch die keine Güter oder Dienstleistungen auf den Kunden übertragen werden, wie bspw. bestimmte vorvertragliche Verwaltungsaufgaben, Vertragseinrichtungsleistungen oder Transportaktivitäten ${ }^{82}$, stellen hingegen keine Leistungsverpflichtungen dar (IFRS 15.25). ${ }^{83}$

Das Erfordernis der Identifizierung von Leistungsverpflichtungen löste nach erstmaliger Veröffentlichung von IFRS 15 zahlreiche Anwendungsfragen aus etwa zur Unterscheidung zwischen Erfüllungstätigkeiten und Leistungsverpflichtungen sowie zur Einstufung einzelner Leistungszusagen als eine Reihe eigenständiger Güter oder Dienstleistungen -, die durch die TRG erläutert wurden. ${ }^{84}$ Dabei wurden auch mögliche Standardanpassungen, wie z. B. die Änderung bzw. Rückeinführung ${ }^{85}$ der Regelung zur Erfassung einer Reihe eigenständiger Leistungszusagen als praktischer Behelf anstatt als verbindliche Regelung, diskutiert, ${ }^{86}$ jedoch im Rahmen der nachträglichen Klarstellung nicht vom IASB adressiert. Die Abgrenzung der zur Vertragserfüllung durchgeführten Tätigkeiten von der für das Vorliegen einer Leistungsverpflichtung notwendigen Übertragung von Gütern oder Dienstleistungen wird indes in einer Agenda-Entscheidung durch das IFRS Interpretations Committee erläutert. ${ }^{87}$ Bspw. Tätigkeiten, die zur Notierung an einer Börse für den Kunden erbracht werden, begründen demnach keine Leistungsverpflichtung; nur die Zulassung selbst stellt eine an den Kunden übertragene Leistung dar. ${ }^{88}$ Bei der Identifizierung von Leistungsverpflichtungen sind

\footnotetext{
${ }^{82}$ Vgl. Upmeier (2018), S. 366-370.

${ }^{83} \mathrm{Vgl}$. IFRS 15.BC93.

${ }^{84}$ Vgl. TRG (2015c), Agenda Paper 25, Rz. 8-11; TRG (2015d), Agenda Paper 27, Rz. 1020; TRG (2015b), Agenda Paper 44, Rz. 33.

${ }^{85}$ Vgl. Re-ED (2011), 30; bereits ED (2010), 24.

${ }^{86}$ Vgl. TRG (2015d), Agenda Paper 27, Rz. 10-20; TRG (2015e), Agenda Paper 34, Rz. 44.

${ }^{87}$ Vgl. IFRS Interpretations Committee (2019a), Agenda Paper 3; Technical Staff (2018e), Agenda Paper 2, Rz. 8-50.

${ }^{88}$ Vgl. IFRS Interpretations Committee (2019a), Agenda Paper 3; Technical Staff (2018e), Agenda Paper 2, Rz. 20-26.
} 
folglich die jeweilige Art der Leistungszusage und die Wesentlichkeit einzelner Leistungszusagen im Vertragskontext zu berücksichtigen. 89

\subsection{Abgrenzung von Leistungsverpflichtungen anhand des Kriteriums der Eigenständigkeit}

\subsection{1 Abstrakte Abgrenzbarkeit durch eigenständige Nutzbarkeit der}

\section{Leistungszusage}

Die Abgrenzung einzelner Leistungsverpflichtungen wird durch die Eigenständigkeit der Leistungszusagen bestimmt, sog. Distinct-Kriterium; nicht eigenständige Leistungszusagen sind daher mit anderen zusammenzufassen und sofern erforderlich auch vertragsübergreifend als eine Leistungsverpflichtung $\mathrm{zu}$ erfassen (IFRS 15.30). Als eigenständig abgrenzbar bzw. „distinct“ gelten Güter oder Dienstleistungen, die der Kunde einzeln oder zusammen mit anderen, für ihn jederzeit zur Verfügung stehenden Ressourcen nutzen kann und die von anderen Leistungszusagen des Vertrags separierbar sind (IFRS 15.27). Während die Abgrenzung von Leistungsverpflichtungen im Discussion Paper an die zu unterschiedlichen Zeitpunkten erfolgende Übertragung von Gütern oder Dienstleistungen geknüpft war, ${ }^{90}$ wurde im Exposure Draft das Kriterium der Eigenständigkeit eingeführt und zunächst an die Einzelverkehrsfähigkeit identischer oder vergleichbarer Güter und Dienstleistungen bzw. die separate Veräußerungsmöglichkeit der jeweiligen Güter und Dienstleistungen, die einen eigenständigen Nutzen und eine eigenständige Gewinnmarge erforderte, geknüpft. ${ }^{91}$ Die überarbeiteten Kriterien im Re-Exposure Draft verlangten für die eigenständige Abgrenzbarkeit der Güter oder Dienstleistungen die regelmäßige Einzelveräußerung durch das Unternehmen oder die Nutzenstiftung für den Kunden; eine Zusammenfassung in Leistungsbündel sollte hingegen bei starker Abhängigkeit der Güter oder Dienstleistungen im Fall der Integration und wesentlichen Modifizierung oder Kundenspezifizierung erfolgen. ${ }^{92}$ Während der Nutzen für den Kunden beibehalten und durch das kumulativ zu erfüllende Kriterium der Separierbarkeit der Leistungszusagen im finalen Standard ergänzt wurde (IFRS 15.27), erfolgte für die zur Beurteilung der Separierbarkeit konkretisierenden Faktoren noch eine nachträgliche Klarstellung. ${ }^{93}$

\footnotetext{
${ }^{89} \mathrm{Vgl}$. IFRS 15.BC85-BC89 f.; IFRS 15.BC116A-BC116E.

${ }^{90} \mathrm{Vgl}$. DP (2008), 3.24.

91 Vgl. ED (2010), 23.

92 Vgl. Re-ED (2011), $28 \mathrm{f}$.

${ }^{93} \mathrm{Vgl}$. IFRS 15.BC116F-BC116Q.
} 
Das erste Kriterium der eigenständigen Nutzbarkeit für den Kunden verweist auf die abstrakte Abgrenzbarkeit der Leistungszusagen (IFRS 15.27 (a)). Demnach müssen die Güter oder Dienstleistungen einzeln oder in Verbindung mit anderen jederzeit verfügbaren Ressourcen dem Kunden einen wirtschaftlichen Nutzen stiften, wobei IFRS 15.28 nicht auf den hauptsächlichen, vom Kunden beabsichtigten Nutzen abstellt, sondern diesen durch eine in wirtschaftlicher Betrachtungsweise nutzengenerierende Verwendung definiert. ${ }^{94}$ Auch die mit dem Nutzen der Leistungszusage in Zusammenhang stehenden verfügbaren Ressourcen sind weit definiert und nicht an den jeweiligen Vertrag geknüpft. ${ }^{95}$ So sind am Markt einzeln verkaufte Güter und Dienstleistungen sowie Ressourcen, die bereits auf den Kunden übertragen wurden oder die er aus anderen Transaktionen erhalten hat, in die Beurteilung einzubeziehen (IFRS 15.28). Der eigenständige Nutzen kann unter anderem mithilfe der noch in den Standardentwürfen für die Abgrenzbarkeit maßgeblichen Einzelverkehrsfähigkeit der Güter und Dienstleistungen nachgewiesen werden (IFRS 15.28), die bei Standardprodukten, wie etwa den in der Telekommunikationsbranche einzeln veräußerten Mobilfunktelefonen oder Routern, regelmäßig vorliegen sollte ${ }^{96}$. Die abstrakte Abgrenzbarkeit wurde vom IFRS Interpretations Committee auch für Immobilienverträge über den Verkauf eines Grundstücks und die Errichtung einer Immobilie diskutiert; sofern der Kunde einerseits die Errichtung des Gebäudes durch ein anderes Unternehmen durchführen lassen und das Land $\mathrm{zu}$ einem anderem Zweck als die Gebäudeerrichtung nutzen könnte und andererseits die Bauleistung auch ohne Grundstücksübertragung ausgeführt werden könnte, sollte die eigenständige Nutzbarkeit demnach gegeben sein. ${ }^{97}$ Inwieweit trotz faktischer Einschränkungen, wie etwa notwendigem Fachwissen oder erforderlichen Kapazitäten, die eigenständige Nutzbarkeit von Gütern oder Dienstleistungen abstrakt angenommen werden kann, ist im Einzelfall vom Unternehmen zu prüfen; anstatt einer rein hypothetischen Beurteilung scheint jedoch vielmehr eine wirtschaftlich sachgerechte Auslegung erforderlich. ${ }^{98}$

\footnotetext{
94 Vgl. Heintges u. a. (2015), S. 576; Schurbohm-Ebneth/Ohmen (2015b), S. 8.

$95 \mathrm{Vgl}$. IFRS 15.BC100.

96 Vgl. Grote u. a. (2012), S. 111; Brücks u. a. (2017), S. 181.

97 Vgl. IFRS Interpretations Committee (2018), Agenda Paper 2D.

98 Vgl. Wüstemann u. a. (2017b), S. 1197 f.
} 


\subsection{2 Konkrete Abgrenzbarkeit durch Separierbarkeit der Leistungszusage im Vertragskontext}

Die Eigenständigkeit von Leistungsverpflichtungen wird neben der abstrakten Einzelbetrachtung der Leistungszusagen durch die konkrete Abgrenzbarkeit einzelner Leistungszusagen ergänzt (IFRS 15.27 (b)). Dieses zweite kumulativ zu erfüllende Kriterium verlangt die Separierbarkeit einer Leistungszusage von anderen vertraglichen Leistungszusagen, mithin die Berücksichtigung des Vertragskontexts. ${ }^{99}$ Die Anforderung der separaten Identifizierbarkeit basiert konzeptionell auf der Beurteilung der einzelnen Vertragsrisiken. ${ }^{100}$ Einzelne Güter oder Dienstleistungen, die aufgrund eines eigenständigen Nutzens zwar abstrakt abgrenzbar sind, deren Erfüllungsrisiko jedoch voneinander abhängt, wie etwa die zur Erfüllung eines Fertigungsauftrags erforderlichen Baumaterialien und Bauleistungen, sind aufgrund fehlender konkreter Abgrenzbarkeit daher als eine Leistungsverpflichtung zu erfassen. ${ }^{101}$ Während als Ausfluss dieser Risikobeurteilung im Re-Exposure Draft bei signifikanten Modifikationsleistungen und wesentlichen Interdependenzen zwischen den Gütern oder Dienstleistungen die Eigenständigkeit der Leistungszusagen nicht gegeben war, ${ }^{102}$ stellen Anpassungsleistungen und das Vorliegen von Abhängigkeiten neben der Durchführung wesentlicher Integrationsleistungen gemäß IFRS 15.29 lediglich Faktoren für die fehlende Eigenständigkeit der Leistungszusagen dar.

Das Kriterium der Eigenständigkeit von Leistungszusagen gegenüber anderen Vertragsleistungen resultierte nach Veröffentlichung von IFRS 15 in zahlreichen Anwendungsfragen an die TRG. ${ }^{103}$ So wurden bspw. unterschiedliche Auffassungen hinsichtlich der Auswirkungen von Lernkurven, der Kundenabsicht und vertraglicher Beschränkungen diskutiert. ${ }^{104}$ Die Ergebnisse der TRG veranlassten das IASB zu einer Klarstellung von IFRS 15.29, die eine definitorische Ergänzung der für die Separierbarkeit im Vertragskontext notwendigen Beurteilung der Leistungszusagen und eine sprachliche Anpassung der Indikatoren aufgrund der nach Ansicht des IASB eindeutigeren Negativabgrenzung der separaten Identifizierbarkeit umfasste, sowie zur Aufnahme weiterer Beispiele. ${ }^{105}$ Zur Beurteilung

\footnotetext{
${ }^{99} \mathrm{Vgl}$. IFRS 15.BC102.

${ }^{100}$ Vgl. IFRS 15.BC103; ED BC (2010), BC56-BC58; Re-ED BC (2011), BC78 f.; Heintges u. a. (2015), S. $576 \mathrm{f}$.

$101 \mathrm{Vgl}$. IFRS 15.B102 f.; IFRS 15.BC116J.

102 Vgl. Re-ED (2011), 29; Re-ED BC (2011), BC78 f.

103 Vgl. TRG (2014), Agenda Paper 9, Rz. 15 f.; Nardmann u. a. (2016), S. 321 f.

104 Vgl. TRG (2014), Agenda Paper 9, Rz. 17-39.

$105 \mathrm{Vgl}$. IFRS 15.BC116F-BC116Q; IFRS 15.IE58A-IE58K; IFRS 15.IE61A.
} 
der separaten Identifizierbarkeit einzelner Leistungszusagen hat ein Unternehmen demnach zwischen der Übertragung einzelner Güter und Dienstleistungen und der Übertragung eines kombinierten Endprodukts zu unterscheiden, wobei nicht die funktionale Abhängigkeit zwischen den einzelnen Gütern und Dienstleistungen entscheidend ist, sondern ihre transformierende Beziehung im Rahmen der Vertragserfüllung. ${ }^{106}$ Sofern vertraglich ein Gesamtwerk geschuldet ist, die Verbindung und Anpassung einzelner Komponenten mithin einen wesentlichen Vertragsbestandteil darstellen, wie häufig bei Fertigungsaufträgen, deutet dies auf die fehlende Eigenständigkeit der Leistungszusagen hin. ${ }^{107}$ Bei vertraglich vereinbarten Standardprodukten und -dienstleistungen sollten hingegen aufgrund fehlender Kundenspezifizierung oder wesentlicher Integrationsleistungen in der Regel abgrenzbare Leistungsverpflichtungen vorliegen. ${ }^{108}$ Auch Leistungszusagen, die lediglich in einem Funktionszusammenhang mit anderen Gütern oder Dienstleistungen stehen, wie bspw. bei produktzugehörigen Verbrauchsmaterialien, sind demnach separierbar. ${ }^{109}$ Abhängigkeiten zwischen Gütern und Dienstleistungen, die das Resultat vertraglicher Gestaltungen sind, etwa die Bindung des Kunden an die vom Unternehmen durchgeführte Standardinstallationsleistung, stehen der Separierbarkeit der Leistungszusage ebenfalls nicht entgegen; maßgeblich ist jedoch die Beurteilung der Eigenschaften der einzelnen Leistungszusagen und inwieweit ihr Erfüllungsrisiko durch die Vertragsstrukturierung beeinflusst wird. ${ }^{110}$ Auch das IFRS Interpretations Committee verdeutlicht im Fall eines Immobilienvertrags über den Verkauf eines Grundstücks und die Errichtung einer Immobilie, dass bei dem in Frage stehenden Fall zwar die Separierbarkeit des Grundstücksverkaufs und des Gebäudebaus vorliegt, es jedoch anstelle einer allgemeinen Entscheidung einer einzelfallspezifischen Beurteilung bedarf. ${ }^{111}$ Die Beurteilung der konkreten Abgrenzbarkeit im Vertragskontext wird daher trotz Klarstellung des IASB und der Erläuterungen des IFRS Interpretations Committee Ermessensentscheidungen von Unternehmen erfordern. ${ }^{12}$

$106 \mathrm{Vgl}$. IFRS 15.BC116K.

${ }^{107}$ Vgl. IFRS 15.BC107 f.; IFRS 15.IE45-IE48; Heintges u. a. (2015), S. 576; SchurbohmEbneth/Ohmen (2015b), S. $8 \mathrm{f}$.

108 Vgl. IFRS 15.IE49-IE53; Brücks u. a. (2017), S. 181.

${ }^{109} \mathrm{Vgl}$. IFRS 15.IE58G-IE58K.

${ }^{110}$ Vgl. IFRS 15.BC116O, IFRS 15.IE58A-IE58F; Heintges u. a. (2015), S. 577.

${ }^{111}$ Vgl. IFRS Interpretations Committee (2018), Agenda Paper 2D; Technical Staff (2017b), Agenda Paper 2 A, Rz. 18-24; zur Diskussion der Abgrenzung von Sonder- und Gemeinschaftseigentum Schoo (2013), S. 73-75.

112 Vgl. IFRS 15.BC105; Nardmann u. a. (2016), S. 322; Wüstemann/Wüstemann (2014), S. 933. 


\subsection{Aufteilung des Transaktionspreises auf eigenständige Leistungsverpflichtungen}

\subsection{Aufteilung des Transaktionspreises auf Basis der relativen Einzelveräußerungspreise}

Die Umsatzerfassung erfolgt bei Erfüllung der Leistungsverpflichtungen in Höhe der hierfür voraussichtlich erhaltenen Gegenleistung (IFRS 15.46). Der Transaktionspreis ist dabei auf eigenständige Leistungsverpflichtungen bzw. eine Reihe eigenständig abgrenzbarer Güter oder Dienstleistungen auf Basis der relativen Einzelveräußerungspreise aufzuteilen (IFRS 15.73 f.). Diese sog. „relative stand-alone selling price basis" gilt seit dem Discussion Paper als anzuwendende Aufteilungsmethode. ${ }^{113}$ Der zu Vertragsbeginn für die jeweiligen Güter oder Dienstleistungen zu bestimmende Einzelveräußerungspreis entspricht dem Preis, zu dem das Unternehmen die Güter oder Dienstleistungen einzeln verkaufen würde (IFRS 15.76 f.). Beobachtbare Preise für den separaten Verkauf der Güter oder Dienstleistungen unter vergleichbaren Umständen und an ähnliche Kunden, wie etwa bei regelmäßig veräußerten Standardprodukten, stellen den besten Anhaltspunkt dar; vertraglich vereinbarte Preise oder festgelegte Listenpreise sind daher nicht automatisch als Einzelveräußerungspreise anzusetzen (IFRS 15.77). Anstelle einer vertragsbasierten Beurteilung erfolgt die Bestimmung des Einzelveräußerungspreises somit unter Berücksichtigung der wirtschaftlichen Substanz. ${ }^{114}$ Sofern der Einzelveräußerungspreis nicht direkt beobachtbar ist, ist er anhand aller vernünftigerweise zur Verfügung stehenden Informationen zu schätzen (IFRS 15.78).

Ein Preisnachlass, der sich aus der Differenz der Summe aller Einzelveräußerungspreise und der im Verhältnis geringeren vereinbarten Gegenleistung ergibt, ist grundsätzlich proportional auf alle Leistungsverpflichtungen aufzuteilen (IFRS 15.81). ${ }^{115}$ Die Ausnahmeregelung von der gesamtvertraglichen Zuordnung in IFRS 15.82 wurde im Re-Exposure Draft eingeführt und knüpft die Aufteilung von Preisnachlässen an die - im Exposure Draft noch für die Vertragssegmentierung relevante - Beurteilung der Preisinterdependenz der Güter und Dienstleistungen. ${ }^{116}$ Auch für variable Vergütungen ist entsprechend der im Re-Exposure Draft aufgenommenen Ausnahmeregelung zu prüfen, ob variable Gegenleistungen dem gesamten Vertrag oder nur einzelnen Leistungsverpflichtungen zuzuordnen sind, wobei wiederum die Abhängigkeit zwischen der Variabilität

\footnotetext{
113 Vgl. DP (2008), 5.45 f.; ED (2010), 50 f.; Re-ED (2011), 70 f.; IFRS $15 . B C 279$ f.

114 Vgl. Heintges u. a. (2015), S. 578; Brune (2016b), S. 22.

${ }^{115} \mathrm{Vgl}$. beispielhaft Brücks u. a. (2017), S. $181 \mathrm{f}$.

116 Vgl. Re-ED BC (2011), BC190 f.
} 
des Entgelts und der Erfüllung der jeweiligen Leistungsverpflichtung zu beurteilen ist (IFRS 15.84 f.). Sofern ein Preisnachlass eine variable Vergütung darstellt, sind zunächst die Regelungen zur Aufteilung variabler Gegenleistungen anzuwenden (IFRS 15.86). ${ }^{117}$

\subsection{Methoden zur Schätzung der Einzelveräußerungspreise}

Für die Schätzung des Einzelveräußerungspreises eines Guts oder einer Dienstleistung werden in IFRS 15.79 beispielhaft der Adjusted-Market-AssessmentAnsatz, der Expected-Cost-Plus-A-Margin-Ansatz und der Residualwertansatz als geeignete Methoden genannt, jedoch weder eine verpflichtende Anwendung noch eine Anwendungshierarchie festgelegt ${ }^{118}$. Bei Anwendung des marktorientierten Ansatzes wird der Einzelveräußerungspreis anhand des Betrags ermittelt, der für die Güter oder Dienstleistungen im jeweiligen Markt erzielt werden könnte oder anhand der Preise von Konkurrenten für ähnliche Güter oder Dienstleitungen, letztere angepasst an unternehmensspezifische Kosten und Gewinnmargen (IFRS 15.79(a)). Der Einzelveräußerungspreis kann aber auch mithilfe des kostenorientierten Ansatzes auf Basis der zur Leistungserfüllung erwarteten (internen) Kosten zuzüglich einer angemessenen Marge geschätzt werden (IFRS 15.79(b)). ${ }^{119}$

Während beide Ansätze bereits im Discussion Paper und Exposure Draft als mögliche Schätzverfahren aufgeführt wurden, wurde der Residualwertansatz erst im Re-Exposure Draft ergänzt. ${ }^{120}$ Der Residualwert entspricht dem Unterschiedsbetrag zwischen dem Transaktionspreis und der Summe der beobachtbaren Einzelveräußerungspreise einzelner Leistungsverpflichtungen (IFRS 15.79(c)) und widerspricht aufgrund seiner Ermittlung als Restwert anstatt als direkt ermittelter Wert insofern den anderen zwei Schätzverfahren. ${ }^{121}$ Die Anwendung des Residualwertansatzes wird im Gegensatz zum markt- und kostenorientierten Ansatz jedoch an Voraussetzungen geknüpft. ${ }^{122}$ So kann nur bei stark variierenden oder unsicheren Veräußerungspreisen der Einzelveräußerungspreis durch den Residualwert abgebildet werden, was vorwiegend für immaterielle Vermögenswerte gelten

117 Vgl. TRG (2015f), Agenda Paper 31, Rz. 7-10; TRG (2015e), Agenda Paper 34, Rz. 7. $118 \mathrm{Vgl}$. IFRS 15.BC286; IFRS 15.BC274.

${ }^{119}$ Vgl. beispielhaft Konold/Müller (2015), S. 6 f.

${ }^{120}$ Vgl. Re-ED BC (2011), BC181 f.

${ }^{121}$ Vgl. Wagenhofer (2014), S. 369.

122 Vgl. Schurbohm-Ebneth/Viemann (2015a), S. 186. 
sollte (IFRS 15.79(c)). ${ }^{123}$ Da der Residualwertansatz der Schätzung des Einzelveräußerungspreises für eigenständige Leistungsverpflichtungen dient, ist ein Wert gleich oder nahe null ausgeschlossen. ${ }^{124}$ Auch Preisnachlässe sind daher vor Ermittlung des Residualwerts im Fall der Zuordenbarkeit zunächst einer Leistungsverpflichtung zuzurechnen (IFRS 15.83). Sofern ein Vertrag mehrere Güter oder Dienstleistungen mit stark variierenden oder unsicheren Preisen beinhaltet, kann dies eine Kombination einzelner Ansätze erfordern (IFRS 15.80). Die Schätzung von Einzelveräußerungspreisen eröffnet daher sowohl aufgrund der frei wählbaren Ansätze als auch insbesondere durch den Residualwertansatz zahlreiche Ermessensspielräume. ${ }^{125}$

\subsubsection{Methodik und Vorgehensweise}

\subsubsection{Untersuchungsmethode}

\subsection{Bedeutungserschließung mithilfe qualitativ-inhaltsanalytischer Verfahren}

Um den Einfluss des rekursiven Verhältnisses zwischen Standardsetzung und Standardanwendung auf die Entwicklung der für Mehrkomponentengeschäfte relevanten Regelungen des IFRS $15 \mathrm{zu}$ ergründen, werden die abgegebenen Unternehmensstellungnahmen und die veröffentlichten Standarddokumente des IASB mithilfe qualitativ-inhaltsanalytischer Verfahren untersucht. Die Inhaltsanalyse dient als Forschungsmethode der systematischen Kategorisierung und Analyse verschiedenster Dokumente, wodurch gültige Rückschlüsse aus dem jeweils untersuchten Text gezogen werden können. ${ }^{126}$ Der Fokus von Textanalysen kann auf formalen Textmerkmalen, wie etwa der Wortanzahl oder dem Auftreten bestimmter Merkmale, oder der Erschließung der zugrundeliegenden Bedeutung liegen. ${ }^{127}$ Qualitative Inhaltsanalysen bezwecken die Ermittlung des Bedeutungsgehalts und sind als regelgeleiteter Interpretationsakt zu verstehen, bei dem durch Auslegung eine Zuordnung von Textstellen und Kategorien

${ }^{123}$ Vgl. IFRS 15.BC271.

${ }^{124} \mathrm{Vgl}$. IFRS 15.BC273.

${ }^{125} \mathrm{Vgl}$. Grote u. a. (2014), S. 412; beispielhaft zu Anwendungsfragen bei Transportdienstleistungen Upmeier (2018), S. 372.

${ }^{126}$ Vgl. Stone u. a. (1966), S. 5; Krippendorff (2013), S. 24-27; Weber (1990), S. 9 f.

${ }^{127}$ Vgl. O'Keefe/Soloman (1985), S. 73; Smith/Taffler (2000), S. 627. 
unter Berücksichtigung einer methodisch kontrollierbaren Textauswertungssystematik erfolgt. ${ }^{128}$ Das Codieren von Textstellen ermöglicht dabei eine Abstraktion der für die Forschungsfrage relevanten Inhalte, ${ }^{129}$ indem die Ausprägungen der einzelnen Kategorien für die jeweiligen Textstellen erfasst werden. ${ }^{130}$

Die Kategorien können deduktiv - aus einer Theorie - hergeleitet, induktiv aus dem Material heraus - erschlossen oder deduktiv-induktiv gebildet werden, also zunächst theoriegeleitet entwickelt und daraufhin am Material angepasst und ergänzt werden. ${ }^{131}$ Als Methoden der kategorienbasierten Analyse sind etwa die inhaltlich strukturierende, die skalierende bzw. evaluative und die typenbildend qualitative Inhaltsanalyse zu unterscheiden, die durch quantitative Auswertungsverfahren, wie z. B. Frequenzanalysen, Valenz- und Intensitätsanalysen sowie Kontingenzanalysen, ergänzt werden können. ${ }^{132}$ So können bspw. mithilfe einer inhaltlich strukturierenden Analyse Themen und Subthemen identifiziert werden und zusätzlich durch Frequenz- und Kontingenzanalysen die Häufigkeit sowie Abhängigkeiten ihres Auftretens untersucht werden. ${ }^{133}$

Die für die Analyse von Rechnungslegungsstandardsetzungsprozessen auf Basis von Stellungnahmen durchgeführten Studien zum Lobbying ${ }^{134}$ lassen sich in Beteiligungsanalysen, in denen die am Prozess beteiligten Interessengruppen und ihre Charakteristika untersucht werden, sowie in Inhalts- und Erfolgsanalysen einteilen, die die inhaltlichen Positionen der Interessengruppen sowie ihren Einfluss auf die Standardsetzung auswerten. ${ }^{135}$ Die Studien bedienen sich dabei inhaltsanalytischer Verfahren, die eher durch ein quantitatives Vorgehen bzw. eine quantitative Auswertung geprägt sind. So wird die Beteiligung der Interessengruppen durch den Anteil der Stellungnehmer untersucht und Kontingenzanalysen

128 Vgl. Mayring (2005), S. 10 f.

${ }^{129}$ Vgl. Gläser/Laudel (2004), S. 44.

130 Vgl. Schreier (2014), S. 2.

${ }^{131}$ Vgl. Mayring (2015), S. 65-99; Kuckartz (2018), S. 63-96.

${ }^{132}$ Vgl. zur Erläuterung der einzelnen Methoden Mayring (2015), S. 65 f., 97-108; Kuckartz (2018), S. 97-161.

133 Vgl. etwa Kuckartz (2018), S. 97-121.

${ }^{134} \mathrm{Vgl}$. etwa folgende insgesamt Puro (1984); Francis (1987); Gavens u. a. (1989); Tandy/Wilburn (1992); Tandy/Wilburn (1996); Saemann (1999); McLeay u. a. (2000); Larson/Brown (2001); Georgiou (2005); Georgiou (2010); Kwok/Sharp (2005); Auste (2011); Giner/Arce (2012); Jorissen u. a. (2012); Jorissen u. a. (2013); Hoffmann/Zülch (2014); Bamber/McMeeking (2016); Shields u. a. (2019).

${ }^{135}$ Vgl. zur Einteilung in Beteiligungs-, Inhalts- und Erfolgsanalysen Auste (2011), S. 4855; zur Unterscheidung der Studien anhand des theoretischen Forschungsansatzes Durocher u. a. (2007), S. 30-32. 
der in Frage stehenden Bilanzierungsthemen durchgeführt ${ }^{136}$ sowie der Erfolg der Stellungnehmer durch die abstrahierte Erfassung der Positionen als Zustimmung oder Ablehnung im Sinne von „votes“137 gemessen ${ }^{138}$. Der Fokus von qualitativen Inhaltsanalysen liegt hingegen auf der Bedeutungserschließung der einzelnen Stellungnahmen und damit auf der Erfassung auch latenter Inhalte und Zusammenhänge. Die Identifizierung verwendeter Argumente oder die Bewertung der Positionen, etwa durch Überprüfung der Stellungnahmen im Hinblick auf den Grad ihrer Ablehnung, verlangen - im Gegensatz zu einer Analyse primär anhand von „qualifiers“ - eine Interpretation der untersuchten Stellungnahmen und folglich ein qualitativ-inhaltsanalytisches Vorgehen. ${ }^{139}$ So kann bspw. die Entscheidungsnützlichkeit eines Regelungsvorschlags durch die Diskussion verschiedener Beispiele in Frage gestellt werden, ohne dass ein expliziter Verweis auf die einzelnen Rahmenkonzeptanforderungen erfolgt. Zudem können Stellungnahmen im Hinblick auf die Zuordnung der Positionen aufgrund der gewählten Formulierungen abweichen, jedoch substanziell die gleiche Regelung präferieren, wie bspw. die Ablehnung eines Regelungsvorschlags mit der Begründung, dass nicht in allen Situationen ein angemessenes Ergebnis erreicht wird, und die Zustimmung zu einem Regelungsvorschlag, mit der Empfehlung eine Ausnahmeregelung für bestimmte Situationen zu ergänzen. ${ }^{140}$

Neben Stellungnahmen werden auch Standardsetzungsdokumente und finale Standards durch ein qualitativ-inhaltsanalytisches Vorgehen untersucht, um Einblicke in die Begründungen des Standardsetzers ${ }^{141}$ und die Umsetzung einer angestrebten Standardausrichtung ${ }^{142}$ zu erlangen. So können im Gegensatz zur quantitativen Erfassung vorgenommener Änderungen mithilfe qualitativer Analysen bspw. die Bedeutungsentwicklung einer Regelung, wie etwa die Anforderung der glaubwürdigen Darstellung, ${ }^{143}$ sowie die Rechtfertigungsstrategie des Standardsetzers $^{144}$ untersucht werden. Die Methode der qualitativen Inhaltsanalyse

\footnotetext{
136 Vgl. etwa Jorissen u. a. (2012), S. 703-717.

137 Vgl. zur Kritik Walker/Robinson (1993), S. 11-15 (auch Zitat, S. 11).

${ }^{138}$ Vgl. etwa Giner/Arce (2012), S. 664 f.

${ }^{139}$ Vgl. O'Keefe/Soloman (1985), S. 73 f. (auch Zitat, S. 73); Yen u. a. (2007), S. 61-63; Bamber/McMeeking (2016), S. 65 f.; Wüstemann (2018), S. 19-22.

${ }^{140} \mathrm{Vgl}$. zum Unterschied zwischen ,substantive“ und „drafting issues“ Walker/Robinson (1993), S. 13-15.

141 Vgl. etwa Young (2006), S. 592-595.

142 Vgl. etwa Bradbury/Schröder (2012), S. 3 f.

143 Vgl. Erb/Pelger (2015a), S. 26-34.

${ }^{144}$ Vgl. Young (2003), S. 628-634; Masocha/Weetman (2007), S. 80-95.
} 
eignet sich daher auch für eine kombinierte Analyse der Stellungnahmen und der Standarddokumente, insbesondere da die Entwicklung der Regelungsvorschriften als diskursive Auseinandersetzung verstanden wird.

\subsection{Diskursanalyse}

Durch die konstruktivistische Betrachtung des Standardsetzungsprozesses soll der „argumentative struggle“145 um die Entwicklung von Rechnungslegungsregeln in den Mittelpunkt der Untersuchung gestellt werden. Die Standardsetzung als sozial strukturierter Prozess basiert auf Annahmen und Werten, die sich im Prozess widerspiegeln und wiederum durch dominante Sprachpraktiken zu einem wesentlichen Bestandteil des Prozesses werden. ${ }^{146}$ Gleichzeitig zeichnet sich er Due Process durch seine politische Komponente und damit der strategischen Einflussnahme der am Prozess Beteiligten aus. Als Dimensionen des Diskurses lassen sich daher der Sprachgebrauch, die Kognition sowie die soziale Interaktion charakterisieren, wobei die Diskursanalyse der Untersuchung dieser Dimensionen sowie ihres Zusammenhangs dient, ${ }^{147}$ mithin gesellschaftliche und politische Effekte von Diskursen identifiziert. Im Rahmen der Diskursforschung ist grundsätzlich zwischen der sog. discourse analysis, die der Analyse von Gesprächssituationen im Sinne eines analytisch-pragmatischen Diskursverständnisses dient, und diskurstheoretischen Ansätzen wie etwa Habermas Diskursethik, die die Idee eines herrschaftsfreien Diskurses als Grundlage eines normativen Modells verfolgt, ${ }^{148}$ und Foucaults Diskurstheorie, die der Untersuchung der Funktion des Diskurses als Sinnproduktion dient, ${ }^{149}$ zu unterscheiden. ${ }^{150}$ Die Diskursanalyse als Untersuchungsperspektive wird in Abhängigkeit der diskurstheoretischen Perspektive für eine Vielzahl unterschiedlicher Forschungsansätze eingesetzt bzw. konkretisiert; neben sprachwissenschaftlichen oder kulturalistischen Untersuchungen finden Diskursanalysen besonders im Rahmen der politikwissenschaftlichen Diskursforschung Anwendung. ${ }^{151}$ So werden etwa mithilfe argumentativer Diskursanalysen diskursive Strukturen in einer Diskussion offengelegt, ${ }^{152} \mathrm{um} \mathrm{zu}$

\footnotetext{
${ }^{145}$ Hajer (1995), S. 53.

146 Vgl. Stenka/Jaworska (2019), S. 3.

147 Vgl. van Dijk (1997), S. 2.

148 Vgl. insgesamt Habermas (1973).

${ }^{149}$ Vgl. insgesamt Foucault (1994).

${ }^{150}$ Vgl. Kerchner/Schneider (2006), S. 9 f.; Keller (2011), S. 13-58.

${ }^{151}$ Vgl. etwa zum Überblick verschiedener Ansätze Keller (2011), S. 13-64; spezifisch zur politischen Diskursanalyse Nonhoff (2006), S. 40-42.

152 Vgl. Hajer (2006), S. 67.
} 
verstehen, wie sich bestimmte Bedeutungen einzelner Problemfelder gegenüber anderen durchsetzen, auf welche Art und Weise diese dargestellt werden und wie Diskurskoalitionen entstehen. ${ }^{153}$

Diskursanalysen bedienen sich je nach Untersuchungsschwerpunkt unterschiedlicher Methoden, beruhen jedoch häufig auf Textanalysen. ${ }^{154}$ Neben formalen Untersuchungen der Diskursbeiträge ist die Analyse von Diskursen meist durch ein qualitativ-inhaltsanalytisches Vorgehen gekennzeichnet, das auch eine Erfassung der rhetorisch-sprachlichen Dimension umfasst. ${ }^{155}$ Die Codierung diskursiver Beiträge zur Erfassung der von Diskursteilnehmern angesprochenen Themen kann etwa durch inhaltsanalytische Kategorien erfolgen, wobei durch die Identifizierung von Gemeinsamkeiten und Unterschieden die Verbreitung verschiedener Deutungen und Argumente zwischen den Akteuren rekonstruiert werden kann. ${ }^{156}$ Die übereinstimmende Verwendung von Argumenten weist insofern auf geteilte Deutungen hin, wohingegen Unterschiede mögliche Konflikte aufzeigen; dies kann Aufschluss ,über die kollektive Reichweite oder den Institutionalisierungsgrad einzelner Deutungsangebote " ermöglichen. ${ }^{157}$

Zur Untersuchung der Diskursetablierung eignet sich zudem eine rhetorische Analyse, die den Fokus auf den Überzeugungsprozess legt. ${ }^{158}$ Durch die Erfassung von „persuasive 'devices'“159 können Einblicke in die „diskursive Erzeugung von Zustimmung“ bzw. Ablehnung erlangt werden. ${ }^{160}$ Unter Rhetorik wird insofern der Einsatz von Überzeugungskraft verstanden, um eine bestimmte Einstellung bzw. ein bestimmtes Verhalten oder Änderungen dieser herbeizuführen, mithin ein Instrument der Kommunikation und Interaktion, das Möglichkeiten der Einflussnahme eröffnet. ${ }^{161}$ Rhetorik lässt sich in Anschluss an Aristoteles anhand der Art der Überzeugung durch Ethos, d. h. Glaubwürdigkeit, die etwa durch den Verweis auf Gemeinsamkeiten und Expertise erlangt wird, Logos, d. h. Rationalität, die bspw. durch eine logische Argumentation und Nachweise oder Beispiele erzeugt wird, und Pathos, d. h. Emotionen, die durch

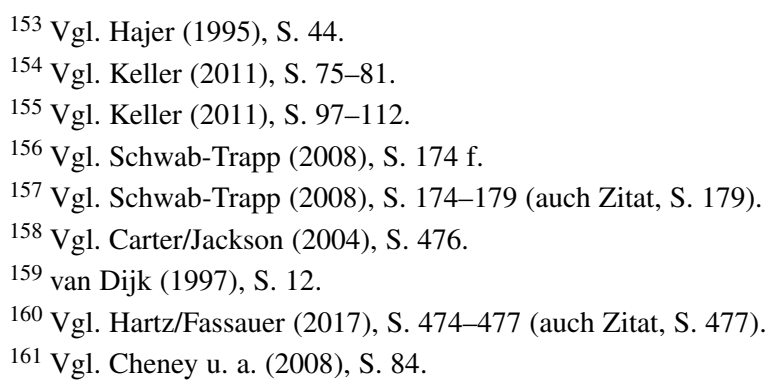


emotionale Appelle erreicht werden, unterscheiden. ${ }^{162}$ Eine rhetorische Analyse umfasst dabei auch eine Untersuchung der verwendeten rhetorischen Stilmittel, wie etwa Metaphern oder Metonymien. ${ }^{163}$

Die diskursanalytischen Studien zur Rechnungslegungsforschung sind durch eine Vielzahl verschiedener theoretischer und methodischer Ansätze gekennzeichnet, ${ }^{164}$ wobei methodisch sowohl quantitative ${ }^{165}$ als auch qualitative ${ }^{166}$ und gemischte ${ }^{167}$ inhaltsanalytische Verfahren angewendet werden. So liegt der Fokus etwa auf der Untersuchung der gesellschaftlichen Bedeutung von Diskursen, wie z. B. im Zusammenhang mit der Etablierung des Rechenschaftszwecks und der Entwicklung des Rahmenkonzepts ${ }^{168}$ oder den ideologischen Auswirkungen von Rechnungslegungsdiskursen ${ }^{169}$. Verschiedene Studien sind zudem durch rhetorische Analysen geprägt, die bspw. durch Analysen von Mythen die Wissensvermittlung in Gesetzgebungsprozessen ergründen ${ }^{170}$ oder durch Metaphernund Kollokationsanalysen von Standardsetzungsdokumenten die Konstruktion des (fiktiven) Nutzers oder die Überzeugungsstrategie des Standardsetzers untersuchen $^{171}$.

Unter Berücksichtigung der in der Rechnungslegungsforschung bestehenden Lobbying- und diskursanalytischen Studien wird in der folgenden Analyse der in der politikwissenschaftlichen Forschung verwendete Ansatz der argumentativen Diskursanalyse ${ }^{172}$ einbezogen. Diskurs wird demnach definiert ,als ein spezifisches Ensemble von Ideen, Konzepten und Kategorisierungen, das sich in einem spezifischen Set sozialer Praktiken produziert, reproduziert und transformiert",

162 Vgl. insgesamt Aristoteles/transl. by H. C. Lawson-Tancred (1991); Aho (1985), S. 23; Cockcroft/Cockcroft (1992), S. 1-8; Green (2004), S. 659 f.; Higgins/Walker (2012), S. 197 f.; Stenka (2014), S. 15; La Torre u. a. (2020), S. 5-7.

163 Vgl. zur Übersicht verschiedener Stilmittel Kallendorf/Kallendorf (1985), S. 36 f.

164 Vgl. für einen Überblick insgesamt Beattie (2014).

$165 \mathrm{Vgl}$. etwa folgende insgesamt Robb/Zarzeski (2001); Cho u. a. (2010).

166 Vgl. etwa folgende insgesamt Young (2003); Masocha/Weetman (2007); Nielsen/Madsen (2009); Duval u. a. (2015); Lupu/Sandu (2017); Stolowy u. a. (2019).

${ }^{167} \mathrm{Vgl}$. etwa folgende insgesamt Bamber/McMeeking (2016); Baudot u. a. (2017).

${ }^{168}$ Vgl. Murphy u. a. (2013), S. 77-87.

${ }^{169}$ Vgl. Zhang/Andrew (2016), S. 3-17.

${ }^{170}$ Vgl. Hoffmann/Zülch (2014), S. 719-721.

${ }^{171} \mathrm{Vgl}$. etwa folgende insgesamt Walters-York (1996); Young (2001); Young (2003); Young (2006); Masocha/Weetman (2007); Walters/Young (2008); Amernic/Craig (2009); Amernic (2013); Young (2013); Stenka/Jaworska (2019).

${ }^{172}$ Vgl. Hajer (1993), S. 44-48; Neumann (2016), S. 288-290; Kurze (2018), S. 99-121. 
wobei bestimmte Diskurse identifiziert werden können, ,die Muster für legitimes Handeln vorgeben und als Wegweiser innerhalb der institutionellen Praktiken fungieren." ${ }^{173}$ Dabei lassen sich von den beteiligten Akteuren verwendete charakteristische story-lines nachweisen, durch die bestimmte Sichtweisen produziert bzw. reproduziert werden und die eine unterschiedliche Anschlussfähigkeit aufweisen. ${ }^{174}$ Story-lines erfüllen demnach die Funktion, die diskursive Komplexität eines Problems zu reduzieren und Möglichkeiten der Problemlösung zu schaffen; sie können sich im Fall der Akzeptanz der Akteure etablieren und zur Rechtfertigung einer spezifischen Herangehensweise für ein scheinbar einheitliches Problem dienen. ${ }^{175}$ Mithilfe von story-lines lassen sich Akteure positionieren sowie bestimmte Ideen und Vorstellungen zuschreiben. ${ }^{176}$ Zudem können Diskurskoalitionen identifiziert werden, die sich durch die gemeinsame Verwendung bestimmter story-lines auszeichnen. ${ }^{177}$ Vor diesem Hintergrund ist ein Bestandteil der Analyse herauszuarbeiten, inwieweit die Etablierung der Regelungsvorschriften für Bilanzierungsprobleme im Zusammenhang mit der Umsatzerfassung aus Mehrkomponentengeschäften von dem Ergebnis diskursiver Interaktion abhängt und Prozesse des Wandels durch die in diesem Zusammenhang stehenden Diskurse beeinflusst werden.

\subsubsection{Kategoriensystem}

\subsection{Methodisches Vorgehen}

Für die Ermittlung des Einflusses von Unternehmen und dem IASB auf die Standardsetzung wurde eine qualitative Inhaltsanalyse durchgeführt, die als bedeutungserschließende Untersuchungsmethode eine systematische Identifizierung von Gemeinsamkeiten und Unterschieden sowie Mustern in dem zu analysierenden Datensatz ermöglicht. Die manuelle Erfassung der Inhalte sowie ihre Auswertung erfolgte dabei computergestützt mithilfe des Programms QDAMiner. ${ }^{178}$ Für die Codierung in QDAMiner wurden die Stellungnahmen und Standardentwürfe in einem ersten Schritt formatiert und mithilfe von Acrobat/Adobe Pro in Textdateien umgewandelt. Anschließend erfolgte die manuelle Aufbereitung der Word-Dateien, wobei insbesondere Fehler der OCR-Texterkennung behoben

173 Vgl. Hajer (1997), S. 111 (auch Zitate, Hervorhebungen im Original).

174 Vgl. Hajer (1997), S. 111.

175 Vgl. Hajer (1995), S. 63-65.

176 Vgl. Hajer (1995), S. 64 f.

177 Vgl. Hajer (1995), S. 65-67.

${ }^{178}$ Vgl. etwa zur Verwendung von QDA-Software Bamber/McMeeking (2016), S. 64 f. 
sowie nicht codierbare Textfelder, wie bspw. Grafiken und Firmenlogos, entfernt wurden. Mithilfe von QDAMiner wurde der aufbereitete Datensatz durch inhaltlich strukturierende Verfahren codiert.

Die Entwicklung der theoretischen Haupt- sowie Subkategorien erfolgte im Rahmen der inhaltlich strukturierenden Analyse deduktiv-induktiv, mithin theoriebasiert auf Basis der bestehenden Literatur und durchgeführten Studien zur Rechnungslegungsstandardsetzung und Unternehmensberichterstattung sowie Ergänzungen am Material im Zuge der Datenanalyse; die thematischen Kategorien wurden induktiv am Material gebildet. ${ }^{179}$ Das Kategoriensystem ist folglich hierarchisch aufgebaut, wobei die inhaltliche Ausrichtung sowie die Rhetorik als übergeordnete Hauptkategorien verwendet und durch Subkategorien unterschiedlicher über- und untergeordneter Ebenen ergänzt wurden. ${ }^{180} \mathrm{Im}$ Rahmen der Codierung wurden Textstellen, die mit einer bestimmten Kategorie in Verbindung stehen, einer vorab gebildeten Kategorie zugeordnet oder in eine dabei generierte Kategorie überführt. ${ }^{181}$ Die Länge bzw. der Umfang einer zu codierenden Textstelle wurde dabei nicht festgelegt, sondern entspricht der der Kategorie zugehörigen Sinneinheit. ${ }^{182}$ Aufgrund der thematisch-analytischen Codierung kann eine Textstelle mit mehr als einer Kategorie in Verbindung stehen, mithin auch mehreren Kategorien zugeordnet worden sein, bspw. weil in einem Textabschnitt mehrere, unterschiedliche Themen adressiert oder unterschiedliche Überzeugungsarten verwendet wurden. ${ }^{183}$ Die Subkategorien der inhaltlichen Zielrichtung zu den Regelungen zur Vertragszusammenfassung sowie zur Aufteilung von Leistungsverpflichtungen und zur Aufteilung des Transaktionspreises dienten der selektiven Beschränkung des Datensatzes auf den aus der Forschungsfrage folgenden Fokus auf Mehrkomponentengeschäfte. ${ }^{184}$ Stellungnahmen, die ausschließlich Themen außerhalb des Forschungsinteresses adressierten und folglich keine Äußerungen zu Bilanzierungsproblemen beinhalteten, die einer der Subkategorien der inhaltlichen Ausrichtung zuordenbar waren, wurden in einer Restkategorie erfasst. ${ }^{185}$

${ }^{179}$ Vgl. zu den unterschiedlichen Kategorien und den Arten der Kategorienbildung Kuckartz (2018), S. 34, 63-96, $101 \mathrm{f}$.

180 Vgl. Kuckartz (2018), S. 38 f.

${ }^{181}$ Vgl. zu Unterschieden des Codierens Strauss/Corbin (1990), S. 61-142.

182 Vgl. Kuckartz (2018), S. 103 f.

183 Vgl. Kuckartz (2018), S. 44, 102 f.

${ }^{184} \mathrm{Vgl}$. zum selektiven Vorgehen und Ausschluss nicht untersuchungsrelevanter Äußerungen Hoffmann/Zülch (2014), S. 715.

185 Vgl. Kuckartz (2018), S. 66 f. 
Die manuelle Codierung erfolgte auf Basis des erstellen Kategoriensystems ${ }^{186}$, d. h. durch Kategorienzuordnung im Rahmen sequenzieller Textdurchsicht, ${ }^{187}$ ohne die Verwendung quantitativer Verfahren, wie etwa durch Stichwortsuche oder Frequenzwörterbücher ${ }^{188}$. Um die im Rahmen dieses qualitativen Vorgehens erforderliche Interpretationsnotwendigkeit zu validieren, wurden als Gütekriterium der Kategorienzuordnung präzise Kategorienbeschreibungen und Codier-Indikatoren festgelegt. ${ }^{189}$ Die Zuverlässigkeit der Codierung wurde durch eine zweifache Textdurchsicht des gesamten Datensatzes auf Basis des erstellen Kategoriensystems optimiert.

Die inhaltlich strukturierende Codierung ermöglichte zudem die Identifikation typischer Muster und Elemente ${ }^{190}$ im Datensatz. Durch die Zusammenfassung bzw. Kombination verschiedener Fälle, die Ähnlichkeiten in bestimmten Ausprägungen in dem auf Basis der codierten Kategorien gebildeten Merkmalsraum aufweisen, ${ }^{191}$ erfolgte eine Typisierung verwendeter story-lines ${ }^{192}$. Für die diskursanalytische Rekonstruktion wurden folglich verschiedene Kategorien in Beziehung zueinander betrachtet und dabei erkennbare Wiederholungen bzw. Verbindungen ,zusammengeführt, über einen roten Faden, eine story-line integriert". ${ }^{193}$ Das induktive Vorgehen wurde von der Forschungsfrage nach der Konstituierung der Rechnungslegungsvorschriften geleitet und daher auf den diskursiven Zielkonflikt zwischen einer konzeptionellen Standardsetzung und einer praxisgerechten Standardanwendung ausgerichtet. Die einzelnen story-lines wurden mithilfe von Ankerbeispielen in Form aussagekräftiger Textauszüge veranschaulicht, um die Transparenz und Nachvollziehbarkeit zu gewährleisten. ${ }^{194}$

\subsection{Formale Zuordnung der Stellungnahmen nach Herkunft und Branchenzugehörigkeit}

Für einen differenzierten Überblick der beteiligten Unternehmen und als formale Anknüpfung für die spätere Auswertung wurde den Stellungnahmen unabhängig

\footnotetext{
186 Vgl. zum Überblick des Kategoriensystems der übergeordneten Kategorien Appendix, II. ${ }^{187}$ Vgl. Kuckartz (2018), S. 102 f.; zum analogen Vorgehen Bamber/McMeeking (2016), S. 65.

188 Vgl. etwa Weber (1990), S. 44-53.

${ }^{189}$ Vgl. Kuckartz (2018), S. 66 f.; zu den Kategorienbeschreibungen Appendix, III.

190 Vgl. Keller (2008), S. 92.

${ }^{191}$ Vgl. zur typenbildenden Inhaltsanalyse Kuckartz (2018), S. 143-148.

192 Vgl. Kurze (2018), S. 177-182.

${ }^{193}$ Vgl. Keller (2008), S. 89 f. (auch Zitat, S. 90, Hervorhebungen im Original).

194 Vgl. Kurze (2018), S. 180.
} 
von der Codierung die Herkunft des Unternehmens sowie die jeweilige Branche zugeordnet. ${ }^{195}$ Beide Charakteristika dienen dazu, die erzielten Ergebnisse im Hinblick auf mögliche Einflüsse institutioneller Faktoren, ${ }^{196}$ wie etwa dem zugrundeliegenden Rechtskreis ${ }^{197}$ oder industriespezifischen Eigenschaften ${ }^{198}$, zu beurteilen. Die Herkunft wurde in Anlehnung an die Comment-Letter-Zuteilung des IASB - abgesehen von der Schweiz sowie Kanada - in Regionen aggregiert erfasst. ${ }^{199}$ Die Erfassung der Schweiz und Kanadas erfolgte separat, da beide Länder ein von der kontinentalen Herkunft abweichendes Rechnungslegungssystem aufweisen. Die vom IASB verwendete Kategorie „Multinationals“ wurde nicht übernommen; die Zuordnung erfolgte in Abhängigkeit des Unternehmenssitzes. Aus diesem Vorgehen ergab sich eine Einteilung in die in Tabelle 3.2 abgebildeten acht Herkunftsregionen.

\section{Tabelle 3.2}

Herkunftseinteilung der Unternehmen

\begin{tabular}{l}
\hline Herkunftseinteilung der Unternehmen \\
\hline - Afrika/Middle East/Indien \\
\hline - Asien \\
\hline - Australien/Neuseeland \\
\hline - Europa \\
\hline - Kanada \\
\hline - Schweiz \\
\hline - US-Amerika \\
\hline - Zentral- und Südamerika
\end{tabular}

Die Einteilung und Abgrenzung einzelner Branchen erfolgte entsprechend der Sektorklassifizierung der Deutschen Börse. ${ }^{200}$ Aus Gründen der Übersichtlichkeit wurde die Branchenzugehörigkeit auf übergeordneter Ebene, den sog. Supersektoren, aggregiert erfasst. So gehören etwa zur Fertigungsindustrie verschiedene Industriezweige, wie z. B. Maschinen-, Gebäude- und Anlagenbau oder auch Transportdienstleistungen. Hieraus folgte eine Einordnung anhand der in Tabelle 3.3 abgebildeten acht Branchen.

195 In QDAMiner wurden die Herkunft und Branche als „Variables“ festgelegt.

196 Vgl. Jorissen u. a. (2013), S. 242-246.

${ }^{197}$ Vgl. etwa Bamber/McMeeking (2016), S. 63.

${ }^{198}$ Vgl. Elbannan/McKinley (2006), S. 614-616.

${ }^{199}$ Vgl. Technical Staff (2009a), Agenda Paper 14 A/119 A, Rz. 3.

${ }^{200}$ Vgl. Deutsche Börse (2019), S. 62-66; Lam (2016), S. 285 f. 
Tabelle 3.3

Brancheneinteilung der Unternehmen

\begin{tabular}{l}
\hline Brancheneinteilung der Unternehmen \\
\hline - Fertigungsindustrie (Industrials) \\
\hline - Pharmaindustrie (Pharma \& Healthcare) \\
\hline - Software und Informationstechnologie (Information \\
Technology) \\
\hline - Telekommunikation (Telecommunication) \\
\hline - Rohstoffe (Basic Materials) \\
\hline - Verbraucherdienstleistungen (Consumer Services) \\
\hline - Verbrauchsgüter (Consumer Goods) \\
\hline - Versorgungswirtschaft (Utilities)
\end{tabular}

\subsection{Kategorienüberblick}

\subsection{Inhaltliche Zielrichtung}

3.1.3.2.3.1.1 Bilanzierungsalternativen für Mehrkomponentengeschäfte

Das für die Inhaltsanalyse verwendete Kategoriensystem wurde aus den Fragestellungen zur inhaltlichen Zielrichtung sowie zur Rhetorik abgeleitet, die die beiden Hauptkategorien darstellen. Die inhaltliche Zielrichtung umfasst dabei zwei Ebenen: Den Inhalt der Regelungsvorschriften, d. h. die möglichen Bilanzierungsalternativen für Mehrkomponentengeschäfte, und die Form der Regelungsvorschriften, d. h. die Umsetzungsmöglichkeiten im Rahmen der Standardausgestaltung. Die thematischen Kategorien für den Inhalt der Regelungsvorschriften wurden aufgrund des Forschungsinteresses an Mehrkomponentengeschäften zunächst durch Fokus auf die Vertragszusammenfassung, die Aufteilung von Leistungsverpflichtungen und die Aufteilung des Transaktionspreises gebildet und durch die in den Standardentwürfen sowie den Stellungnahmen hierzu geäußerten Bilanzierungslösungen konkretisiert (Tabelle 3.4). Während des Standardsetzungsprozesses wurden als Bilanzierungsalternativen in Bezug auf die Zusammenfassung von Verträgen auch die Portfoliobetrachtung sowie die Aufteilung eines Vertrags in mehrere Einzelverträge diskutiert, die im Rahmen der Kategorienbildung daher unter der Kategorie der Ausnahmen vom einzelvertragsbasierten Ansatz, subsumiert wurden.

Als Bilanzierungsalternativen für die Aufteilung von Leistungsverpflichtungen wurden sieben Subkategorien identifiziert. Zusätzlich zur Kategorie der Anknüpfung an den Erfüllungszeitpunkt sowie der Kategorie für Äußerungen, die eine vertragsübergreifende Beurteilung adressierten, wurden das Distinct-Kriterium sowie mit diesem im Zusammenhang stehende Kriterien als Kategorien festgelegt. Obwohl die Beurteilung der Interdependenz, der Risiken, der Gewinnmarge 
und der Einzelveräußerung von Leistungsverpflichtungen in Verbindung mit dem Distinct-Kriterium steht, wurden alle Kriterien als separate Kategorien erfasst, um ein differenziertes Verständnis für die Konkretisierung des Kriteriums der Eigenständigkeit zu erlangen. Die einzelnen Kategorien wurden indes nicht unter dem Distinct-Kriterium subsumiert, da das Kriterium auch ohne den Bezug zu den einzelnen Faktoren adressiert werden konnte. Eine Überschneidung der Kategorien ist mithin möglich.

Die Aufteilung des Transaktionspreises umfasst neben der Kategorie des relativen Einzelveräußerungspreises drei weitere Kategorien für die im Standardsetzungsprozess diskutierten Bewertungsansätze. Während die ContingentRevenue-Cap-Methode und die Residualwertmethode als in der Standardsetzung und Praxis relevante Methoden als eigenständige Kategorien abgegrenzt wurden, erfolgte eine aggregierte Erfassung der sonstig adressierten alternativen Bewertungsmaßstäbe.

Tabelle 3.4 Subkategorien der Kategorie „Bilanzierungsalternativen für Mehrkomponentengeschäfte"

\section{Bilanzierungsalternativen für Mehrkomponentengeschäfte}

- Ausnahmen vom einzelvertragsbasierten Ansatz

- Aufteilung eines Vertrags in mehrere Einzelverträge

- Portfoliobetrachtung

- Zusammenfassung von Verträgen

- Aufteilung von Leistungsverpflichtungen

- Anknüpfung an den Erfüllungszeitpunkt

- Distinct-Kriterium

- Einzelveräußerung

- Interdependenz

- Gewinnmarge

- Risiken

- Vertragsübergreifende Beurteilung

- Aufteilung des Transaktionspreises

- Alternativer Bewertungsmaßstab

- Contingent-Revenue-Cap-Methode

- Relativer Einzelveräußerungspreis

- Residualwertmethode

- Restkategorie 
Die Kategorien für die Bilanzierungsalternativen für Mehrkomponentengeschäfte ermöglichen das Bilanzierungsverständnis der am Standardsetzungsprozess Beteiligten zu erfassen. Im Gegensatz zu evaluativen Kategorien, die bspw. eine Positionierung der Beteiligten anhand der Reichweite ihrer Zustimmung oder Ablehnung vornehmen, bilden die thematischen Kategorien unterschiedliche Bedeutungsgehalte $a b$, mithilfe derer die Reichweite des Einflusses und folglich der Etablierung einzelner Regelungen nachvollzogen werden kann. Stellungnahmen, die keine dieser Kategorien adressierten, wurden daher in einer Restkategorie erfasst, demzufolge auch nicht im Hinblick auf die Kategorien der Umsetzungsmöglichkeiten und der Rhetorik untersucht.

\subsection{2 Umsetzungsmöglichkeiten im Rahmen der Standardausgestaltung}

Die inhaltliche Ausrichtung umfasst neben dem Inhalt der Regelungsvorschriften auch die Form der Regelungsvorschriften. Der Fokus liegt hierbei auf den Umsetzungsmöglichkeiten im Rahmen der Standardausgestaltung und knüpft insofern an die Frage einer prinzipienorientierten oder regelbasierten Standardsetzung an. Die Kategorienbildung erfolgte daher zunächst theoriebasiert. Während eine regelorientierte Standardsetzung durch die Entwicklung eines branchenspezifischen, sehr detaillierten und möglichst jegliche Bilanzierungssachverhalte umfassenden Regelwerks gekennzeichnet ist, ist eine prinzipienorientierte Standardsetzung von allgemeingültigen Prinzipien geprägt, die unter Anwendung sachgemäßen Ermessens der Unternehmen die Bilanzierung unterschiedlicher Sachverhalte abdecken. ${ }^{201}$ Der Fokus der Kategorienbildung für die Umsetzungsmöglichkeiten lag demzufolge auf dem Umfang und der Verbindlichkeit der Regelausgestaltung (Tabelle 3.5). Die Detailliertheit der Regelungen bezieht sich sowohl auf Beispiele und Anwendungsleitlinien als auch auf die Verständlichkeit des Standardtexts und wird von der Kategorie Umfang der Guidance erfasst. Die Verbindlichkeit einer Regelungsvorschrift wurde als industriespezifische Ausnahmeregelung und als Determiniertheit der Regelungsvorschriften kategorisiert, die die Bestimmtheit der Regelungen, wie etwa die Ausprägung von Wahlrechten, umfasst. Die drei Subkategorien wurden im Rahmen der Datenanalyse durch die Kategorie der Übernahme alter Regelungsvorschriften ergänzt, die als thematische Kategorie nur mittelbar an die Prinzipien- oder Regelorientiertheit der Standardsetzung anknüpft, jedoch eine Umsetzungsmöglichkeit für die inhaltliche Standardausgestaltung der Bilanzierungsalternativen darstellt.

${ }^{201}$ Vgl. Schipper (2003), S. 62-71; Benston u. a. (2006), S. 167-171; Wüstemann/Wüstemann (2010), S. 14-21. 
Tabelle 3.5 Subkategorien der Kategorie „Umsetzungsmöglichkeiten im Rahmen der Standardausgestaltung“

\section{Umsetzungsmöglichkeiten im Rahmen der} Standardausgestaltung

- Determiniertheit der Regelungsvorschriften

- Industriespezifische Ausnahmeregelung

- Umfang der Guidance

- Übernahme alter Regelungsvorschriften

\subsection{Rhetorik}

\subsection{1 Ethos: Erzeugung von Glaubwürdigkeit}

Die Anschlussfähigkeit einer Problemlösungsalternative wird nicht allein von ihrer inhaltlichen Zielrichtung, sondern auch von ihrer Überzeugungskraft bestimmt, weshalb neben der inhaltlichen Dimension auch die rhetorische Dimension des Standardsetzungsprozesses analysiert wird. Rhetorik stellt daher die zweite Hauptkategorie dar und umfasst die unterschiedlichen, dennoch zusammenwirkenden Überzeugungsarten, d. h. die „,means of persuasion“ zur Erzeugung von Glaubwürdigkeit (Ethos), Emotionen (Pathos) und Rationalität (Logos), sowie die stilistischen Mittel, die das „stylistic repertoire“ darstellen. ${ }^{202}$ Die Kategorienbildung erfolgte theoriebasiert in Anlehnung an die in existierenden Studien zur Untersuchung der Rhetorik in der Unternehmensberichterstattung verwendeten Kategorien, ${ }^{203}$ die durch Kategorien auf Basis der Literatur zur Rechnungslegungsstandardsetzung und durch Bildung thematischer Kategorien am Material ergänzt wurden.

Die übergeordnete Subkategorie Ethos beinhaltet die Kategorien zur Überzeugung durch Glaubwürdigkeit, die auf dem Charakter und der ,selfpresentation“ 204 der sich äußernden Beteiligten gründet. ${ }^{205}$ Ethos bezieht sich demnach auf die ",personality“ des Autors, etwa in Form des persönlichen Images, des Charismas und seiner Expertise, und dessen „stance“, d. h. seiner Stellung und Grundhaltung sowie der Vermittlung dieser. ${ }^{206}$ Die Erzeugung von Glaubwürdigkeit zur Überzeugung der Adressaten beruht auf der Annahme, dass die Akzeptanz einer Äußerung in Zusammenhang mit dem Vertrauen in den und

\footnotetext{
202 Vgl. Cockcroft/Cockcroft (1992), S. 3 (auch Zitat), S. 114 f. (auch Zitat, S. 115).

${ }^{203}$ Vgl. Higgins/Walker (2012), S. 197 f.; La Torre u. a. (2020), S. 5-7.

${ }^{204}$ Warnock (1992), S. 183.

205 Vgl. Higgins/Walker (2012), S. 197.

206 Vgl. Cockcroft/Cockcroft (1992), S. 19-23.
} 
dem Respekt vor dem Autor steht. ${ }^{207}$ Im Rahmen des Standardsetzungsprozesses geht es folglich um die Erlangung von Glaubwürdigkeit hinsichtlich der Rolle, Positionierung und Charakterisierung des Unternehmens respektive des Standardsetzers.

Die Überzeugungsart Ethos wurde anhand von sechs Subkategorien operationalisiert (Tabelle 3.6). Die Kategorie Gemeinsamkeiten beschreibt die Erzeugung von Glaubwürdigkeit durch das Aufzeigen von Ähnlichkeiten sowie geteilten Hintergründen, Zielen und Werten zwischen dem Autor und den Adressaten, was eine Identifikation ermöglicht und Einigkeit nahelegt. ${ }^{208}$ Sie umfasst dabei auch die Verwendung von Schmeicheleien, die den Autor sympathisch wirken lassen. ${ }^{209}$ Durch die Bezugnahme auf Adressaten bzw. ihre Ansichten sowie durch Aufforderung der Adressaten, ihre Meinung zu äußern, wird Freundlichkeit und Anerkennung vermittelt, was als Respekt kategorisiert wurde. ${ }^{210}$ Glaubwürdigkeit wird zudem durch die Expertise eines Autors in Bezug auf das spezifische Thema erreicht, wobei die Anknüpfung an Qualifikationen, Erfahrungen und Fachwissen überzeugend wirkt. ${ }^{211}$ Die Kategorie der Autorität umfasst sowohl die Vermittlung eigener Autorität, bspw. durch den Bezug auf den eigenen Vorschlag, persönliche Treffen zwischen Autor und Adressaten und Branchentreffen zwischen verschiedenen Autoren, als auch fremder Autorität, etwa durch den Verweis auf andere Institutionen oder den Verweis auf andere Standards. ${ }^{212}$ Durch den Bezug auf die (ökonomische) Realität und die Darstellung der Vermögensund Ertragslage wird an ein Objekt ,out there“ angeknüpft, ${ }^{213}$ weshalb beide Kategorien ebenfalls unter der Kategorie der Autorität erfasst wurden.

\footnotetext{
207 Vgl. Beason (1991), S. 326.

${ }^{208}$ Vgl. Beason (1991), S. 331-333; Allen/Caillouet (1994), S. 48; Higgins/Walker (2012), S. 198; La Torre u. a. (2020), S. 6.

${ }^{209}$ Vgl. Allen/Caillouet (1994), S. 48; zur Subsumtion unter der Kategorie Gemeinsamkeiten La Torre u. a. (2020), S. 5 f.

${ }^{210}$ Vgl. Beason (1991), S. 333-335; Higgins/Walker (2012), S. 198; La Torre u. a. (2020), S. 6, 10 .

${ }^{211}$ Vgl. Beason (1991), S. 337 f.; Higgins/Walker (2012), S. 198; La Torre u. a. (2020), S. 6, 10.

212 Vgl. zur Kategorie der Autorität unter Ethos La Torre u. a. (2020), S. 6.

${ }^{213}$ Vgl. Young (2003), S. 628 (auch Zitat, Hervorhebung im Original).
} 
Tabelle 3.6 Subkategorien der Kategorie „Ethos:

Erzeugung von Glaubwürdigkeit"“

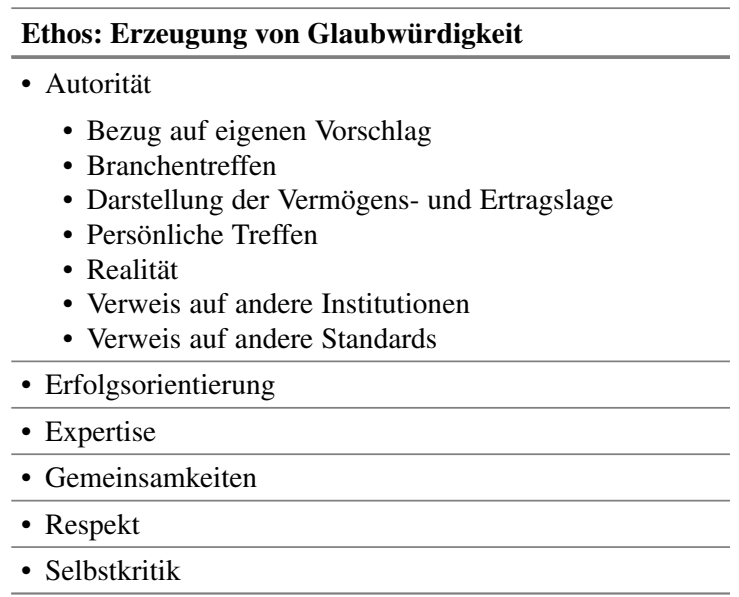

Als weitere Kategorien wurden die Erfolgsorientierung, wonach auf vergangene Leistungen oder künftige Erfolge des Autors aufmerksam gemacht wird, und die Selbstkritik festgelegt, die auf der Vermittlung von Glaubwürdigkeit durch Zugeständnisse eigener Fehler oder Defizite beruht. ${ }^{214}$

\subsection{2 Pathos: Erzeugung von Emotionen}

Die Überzeugungsart Pathos gründet auf der Erzeugung von Emotionen beim Adressaten. Der Fokus liegt auf dem emotionalen Engagement, das auf die Bedürfnisse und Gefühle der Adressaten gerichtet ist und das Verständnis dieser durch den Autor vermittelt. ${ }^{215}$ Emotionales Engagement erfordert dabei ,orientation“, d. h. die emotionale Orientierung bzw. Ausrichtung der Äußerung, sowie ,,actualisation“, d. h. die Umsetzung bzw. die Art, wie Adressaten durch Emotionen überzeugt werden, etwa durch eine bildhafte Sprache oder die Anknüpfung an soziale sowie kulturelle Werte. ${ }^{216}$ In einzelnen Studien zur Untersuchung der Unternehmensberichterstattung wird Pathos durch Subkategorien in Form

${ }^{214}$ Vgl. Beason (1991), S. 335-340; Higgins/Walker (2012), S. 198; La Torre u. a. (2020), S. 6.

${ }^{215}$ Vgl. Higgins/Walker (2012), S. 198; La Torre u. a. (2020), S. 6.

216 Vgl. Cockcroft/Cockcroft (1992), S. 40-50 (auch Zitate, S. 40); Higgins/Walker (2012), S. 198; La Torre u. a. (2020), S. 6 f., 11 f. 
von rhetorischen Figuren unterteilt. ${ }^{217}$ Im Gegensatz hierzu wurden die Stilmittel jedoch als sprachliche Umsetzung der rhetorischen Strategie verstanden, die insofern unterschiedlichen Überzeugungsarten dienen können. Es erfolgte daher eine separate Kategorisierung der rhetorischen Stilmittel und folglich keine Untergliederung der Kategorie Pathos.

\subsection{3 Logos: Erzeugung von Rationalität}

Die Überzeugung durch Logos umfasst als Mittel die Argumentation und kann durch die Ebenen ,invention“, d. h. die Entwicklung von Argumenten zu einem bestimmten Thema, und ,judgement", die die anschließende Abwägung dieser betrifft, charakterisiert werden. ${ }^{218}$ Logos legt den Fokus demnach auf den Gegenstand der Diskussion anstatt auf die Teilnehmenden ${ }^{219}$ und knüpft folglich an die Rationalität und Widerspruchsfreiheit eines Arguments $a^{220}$. Die Rationalität einer Begründung basiert für Zwecke der Rhetorik dabei nicht zwingend auf den gleichen Ansprüchen bzw. Beweispflichten wie die der Logik, um überzeugend zu sein, sondern kann vielmehr auf dem Anschein von Rationalität gründen. ${ }^{221}$ Maßgeblich ist hierfür die Schlüssigkeit einer Argumentation: Während die Verwendung von Fakten, quantitativen Daten und empirischen Nachweisen die Unbefangenheit und Stichhaltigkeit adressiert und eine logische Schlussfolgerung ermöglicht, beruht die Überzeugungskraft abstrakter und ungenauer Konzepte sowie das Fehlen etwaiger Nachweise auf einer „commonsense“222 Schlüssigkeit. ${ }^{223}$

Die Operationalisierung der Überzeugungsart Logos erfolgte zunächst theoriebasiert anhand der in Lobbying-Studien verwendeten Einteilung in konzeptionelle Gründe und ökonomische Gründe (Tabelle 3.7). ${ }^{224}$ Die Kategorie der konzeptionellen Gründe umfasst Argumente, deren Schlüssigkeit auf Rechnungslegungsprinzipien und theoretischen Konzepten gründet. ${ }^{225}$ Als Begründungsmaßstab für die einzelnen Bilanzierungsalternativen kann insofern das Rahmenkonzept

\footnotetext{
${ }^{217}$ Vgl. etwa Higgins/Walker (2012), S. 198; La Torre u. a. (2020), S. 6.

${ }^{218}$ Vgl. Cockcroft/Cockcroft (1992), S. 58 f.

${ }^{219}$ Vgl. Warnock (1992), S. 183.

${ }^{220} \mathrm{Vgl}$. Holt/Macpherson (2010), S. 26.

${ }^{221}$ Vgl. Holt/Macpherson (2010), S. 26; Higgins/Walker (2012), S. 198.

222 Higgins/Walker (2012), S. 198.

${ }^{223}$ Vgl. Nørreklit (2003), S. 593-596; La Torre u. a. (2020), S. 7.

${ }^{224}$ Vgl. zur Einteilung in konzeptionelle und ökonomische Gründe Tutticci u. a. (1994), S. 94-98; Giner/Arce (2012), S. 670-674; Wüstemann (2018), S. 15 f.

225 Vgl. Stenka/Taylor (2010), S. 118.
} 
verwendet werden. ${ }^{226}$ Auf Basis der im Rahmenkonzept verankerten Zwecksetzung und qualitativen Merkmale wurden im Zuge der Datenanalyse daher die Entscheidungsnützlichkeit, die glaubwürdige Darstellung, die Relevanz, die Vergleichbarkeit, die Verlässlichkeit und die Verständlichkeit als Subkategorien identifiziert. Zudem wurde die konsistente Ausgestaltung der Regelungsvorschriften mit der Subkategorie der Konsistenz festgelegt. In der Kategorie der ökonomischen Gründe beruht die Schlüssigkeit der Argumente hingegen auf den ökonomischen Folgen der Bilanzierungsalternativen. ${ }^{227}$ Als Subkategorien wurden daher die Praktikabilität, die Kosten und der Nutzen festgelegt.

Neben den Kategorien der konzeptionellen und ökonomischen Gründe wurde zudem die Verwendung von Beispielen als eigenständige Kategorie erfasst, da Beispiele bzw. beispielhafte Erläuterungen als Nachweise fungieren, mithin der Überzeugung durch Rationalität dienen. ${ }^{228}$

Tabelle 3.7 Subkategorien der Kategorie „Logos: Erzeugung von Rationalität“"

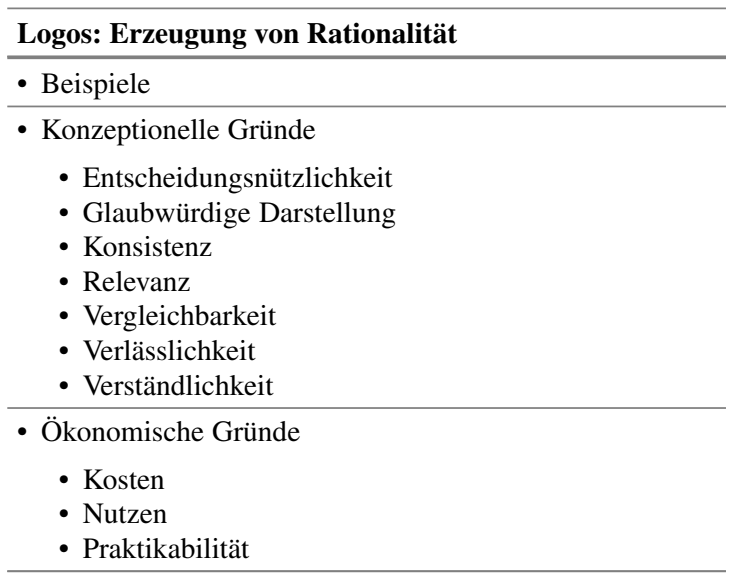

\subsection{4 Rhetorische Stilmittel}

Während die Unterscheidung in Ethos, Pathos und Logos an die Überzeugungsart anknüpft, bezieht sich das ,persuasive repertoire“ auf die linguistische

\footnotetext{
226 Vgl. etwa Tutticci u. a. (1994), S. 94.

227 Vgl. Stenka/Taylor (2010), S. 118.

${ }^{228}$ Vgl. zum ,examples-based reasoning“ im Rahmen der Standardanwendung Clor-Proell/Nelson (2007), S. 700 f.; Capps u. a. (2017), S. 583.
} 
Komponente des Überzeugungsprozesses. ${ }^{229}$ Zur Darstellung eines individuellen Gedankens wird ein Autor den Sprachstil bzw. die Ausdruckweise verwenden, die für die jeweilige Situation, die Adressaten sowie die Nachricht angemessen scheint. $^{230}$ Als Mittel fungieren dabei die Wortwahl und das Lautmuster sowie rhetorische Figuren und der schematische Aufbau, wobei die letztgenannten als „figures of rhetoric“231 in der Analyse durch die Kategorie der rhetorischen Stilmittel erfasst wurden. Diese rhetorischen Figuren ermöglichen es einem Autor, Ideen und Gedanken auf eine Art und Weise zu formulieren und zu präsentieren, die die Glaubwürdigkeit des Autors, die Emotionen der Adressaten und/oder die Rationalität der Argumentation bedienen. ${ }^{232}$

Die Kategorisierung der rhetorischen Stilmittel erfolgte theoriebasiert in Einklang mit bestehenden Studien zur Untersuchung der Rhetorik von Rechnungslegungsstandardsetzern, ${ }^{233}$ wonach als relevante rhetorische Figuren die Metapher und die Metonymie identifiziert wurden (Tabelle 3.8). Metaphern rufen als bildliche Gegenüberstellung Assoziationen hervor, die Emotionen transportieren, abstrakte Konzepte greifbar machen und Themen abstrahieren, ${ }^{234}$ wie bspw. die vom FASB in verschiedenen Rechnungslegungsstandards verwendeten „risk“-Metaphern ${ }^{235}$ oder die metaphorische Konstruktion der ,reporting entity “236. Metaphern sind insofern imstande Diskurse im Standardsetzungsprozess zu gestalten. ${ }^{237}$ Auch durch Metonymien erfolgt eine Abstraktion, jedoch in Form einer Reduktion auf Teile eines Ganzen, d. h. „eine Substitution von Begriffen, die durch eine materielle oder logische Beziehung bestimmt werden."238 So ermöglichen etwa die Metonymie der ,public interest“ oder die metonymische Beschreibung des Standardsetzer als „the Board“ eine kollektive Identifikation bzw. die Vermittlung einer kollektiven Autorität. ${ }^{239}$ Im Rahmen der Datenanalyse wurden der Verweis auf die User als ,,assumed audience of

${ }^{229}$ Vgl. Cockcroft/Cockcroft (1992), S. 114 f. (auch Zitat, S. 114).

${ }^{230} \mathrm{Vgl}$. Cockcroft/Cockcroft (1992), S. 114.

${ }^{231}$ Vgl. Cockcroft/Cockcroft (1992), S. 115-136 (auch Zitat, S. 125).

232 Vgl. Kallendorf/Kallendorf (1985), S. 43.

${ }^{233} \mathrm{Vgl}$. folgende insgesamt Young (2003); Masocha/Weetman (2007); Stenka (2013); Stenka/Jaworska (2019).

${ }^{234}$ Vgl. Walters-York (1996), S. 54-58.

235 Vgl. Young (2001), S. 611-618 (auch Zitat, S. 611).

236 Vgl. Amernic (2013), S. $86-89$ (auch Zitat, S. 86).

${ }^{237}$ Vgl. Walters/Young (2008), S. 811-829.

${ }^{238}$ Vgl. Sillince/Barker (2011), S. 10 (auch Zitat, Übersetzung der Verfasserin).

${ }^{239}$ Vgl. Masocha/Weetman (2007), S. 85 (auch Zitate). 
financial statements“240 sowie die Verwendung von Most/Some zur Beschreibung der Adressaten bzw. der am Standardsetzungsprozess Beteiligten als Metonymien identifiziert und als Subkategorien festgelegt. ${ }^{241}$ Als weitere rhetorische Stilmittel wurden die Antithese, durch die gegensätzliche Ideen bzw. Aussagen miteinander verbunden werden, ${ }^{242}$ die Hyperbel als Übertreibung und die Wiederholung ${ }^{243}$ sowie die rhetorische Frage, durch die eine Zustimmung oder Ablehnung impliziert wird, ${ }^{244}$ als einzelne Subkategorien erfasst.

Tabelle 3.8 Subkategorien der Kategorie „Rhetorische Stilmittel“

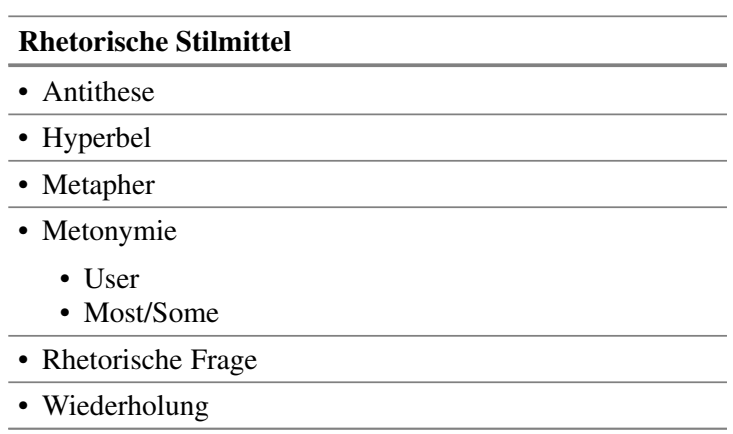

\subsubsection{Datensatzspezifische und methodische Einschränkungen}

Die durchgeführte Analyse unterliegt aufgrund des gewählten Datensatzes sowie der Methodik einzelnen Einschränkungen. Durch die Begrenzung der Datenbasis auf Stellungnahmen ${ }^{245}$ und Standardentwürfe werden andere Formen der diskursiven Auseinandersetzung, wie etwa zwischen den Board- oder Staff-Mitgliedern,

\footnotetext{
240 Young (2003), S. 629.

${ }^{241}$ Vgl. Stenka (2014), S. 18 f.

${ }^{242}$ Vgl. Kallendorf/Kallendorf (1985), S. 36; Masocha/Weetman (2007), S. 86 f.; Stenka (2014), S. 21.

${ }^{243}$ Vgl. Cockcroft/Cockcroft (1992), S. 131-133.

${ }^{244}$ Vgl. Kallendorf/Kallendorf (1985), S. 37.

${ }^{245}$ Vgl. etwa kritisch zu den Limitationen einer Analyse auf Basis von Stellungnahmen Königsgruber (2009), S. 1318; Camfferman/Zeff (2018), S. 302 f.
} 
die bspw. in Form von Interviews ${ }^{246}$ mit den Beteiligten, Audioaufzeichnungen $^{247}$ dieser Treffen oder durch Staff-Paper ${ }^{248}$ untersucht werden könnten, nicht berücksichtigt. Auch der Einfluss von informellen Treffen zwischen den am Standardsetzungsprozess Beteiligten und den Standardsetzern, die unter Ausschluss der Öffentlichkeit stattfinden, ${ }^{249}$ kann anhand der gewählten Datenbasis nicht abgebildet werden. Die Eingrenzung der Analyse auf Stellungnahmen von Unternehmen stellt eine weitere Einschränkung dar, da nicht der Einfluss aller am Standardsetzungsprozess beteiligten Akteure untersucht wurde und hierdurch mögliche Diskurselemente nicht abgebildet werden. Die Analyse umfasst insofern „only a limited extract of reality“, der jedoch nicht willkürlich festgelegt, sondern systematisch aus der Forschungsfrage und den hieraus abgeleiteten Fragestellungen hergeleitet wurde. ${ }^{250}$

Methodische Einschränkungen bestehen aufgrund der der qualitativen Inhaltsanalyse immanenten subjektiven Komponente. Sowohl die Kategorienbildung als auch die Codierung des Materials basiert auf dem eigenen Vorverständnis und der individuellen Durchführung, weshalb Fehler bei der manuellen Codierung nicht ausgeschlossen werden können. Das qualitativ-inhaltsanalytische Vorgehen ist methodisch jedoch etabliert, ${ }^{251}$ besonders wenn eine inhaltliche Erkenntnisgewinnung statt einer quantitativen Erkenntnisverifizierung angestrebt wird. Die Objektivität der Analyse wird durch ihre Nachvollziehbarkeit erreicht, die weniger die Ermittlung einer Intercoder-Übereinstimmung ${ }^{252}$ als vielmehr eine präzise Kategorienbeschreibungen erfordert. Auf Basis des dokumentierten Kategoriensystems sowie des öffentlich verfügbaren Datensatzes kann die Analyse demnach repliziert werden. ${ }^{253}$

Die Analyse ist neben der gewählten Datenbasis in Form von Unternehmensstellungnahmen und der verwendeten Methodik in Form der qualitativen Inhaltsanalyse zudem auf die Regelungsvorschriften des IFRS 15 beschränkt, was die Generalisierbarkeit der Analyseergebnisse per se einschränkt. ${ }^{254}$ Die im

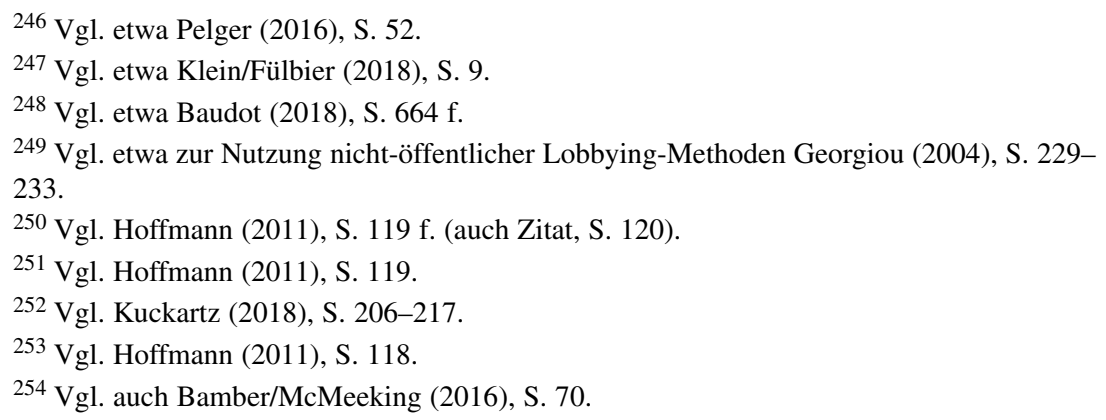


Rahmen der Auswertung gewonnenen Erkenntnisse sowie die darauf basierenden Rückschlüsse können jedoch auf andere Standardsetzungsprozesse übertragen werden und hierdurch Verallgemeinerbarkeit erlangen.

\subsection{Darstellung und Diskussion der Ergebnisse}

\subsubsection{Deskriptiver Überblick untersuchter Stellungnahmen}

Die Auswertung der Analyseergebnisse erfolgt dreischrittig; sie beinhaltet einen Überblick über die Unternehmensbeteiligung der einbezogenen Stellungnahmen, eine Beurteilung der inhaltlichen Zielrichtung und der Rhetorik sowie die Rekonstruktion der relevanten story-lines. Die untersuchten Stellungnahmen stammen von 384 Unternehmen, ${ }^{255}$ die in vier Kommentierungsphasen insgesamt 512 Stellungnahmen abgegeben haben, was einer relativen Beteiligung von 31,35\% entspricht. ${ }^{256}$ Entgegen der theoretischen Annahme, dass eine Beteiligung zu Beginn des Standardsetzungsprozesses aufgrund der beim Standardsetzer noch nicht verfestigten ,preliminary views“ ${ }^{\text {“257 }}$ effektiver ist, ${ }^{258}$ bestätigen sowohl die absolute Gesamtbeteiligung als auch die absolute Unternehmensbeteiligung während der einzelnen Kommentierungsphasen die Ergebnisse von Beteiligungsstudien zu Rechnungslegungsstandardsetzungsprozessen, wonach eine geringere Teilnahme zu Beginn des Prozesses als in späteren Phasen festzustellen ist (Abbildung 3.1). ${ }^{259}$

\footnotetext{
${ }^{255}$ Vgl. zur Übersicht aller teilnehmenden Unternehmen in den einzelnen Kommentierungsphasen Appendix, I.

256 Vgl. Tabelle 3.1.

257 Vgl. etwa DP (2008), S3 f. (auch Zitat, S3).

258 Vgl. Sutton (1984), S. 88 f.

${ }^{259}$ Vgl. Georgiou (2004), S. 225-228; Kurz (2009), S. 172 f.; Georgiou (2010), S. 108 f.; zu einem abweichenden Ergebnis Giner/Arce (2012), S. 666-670.
} 


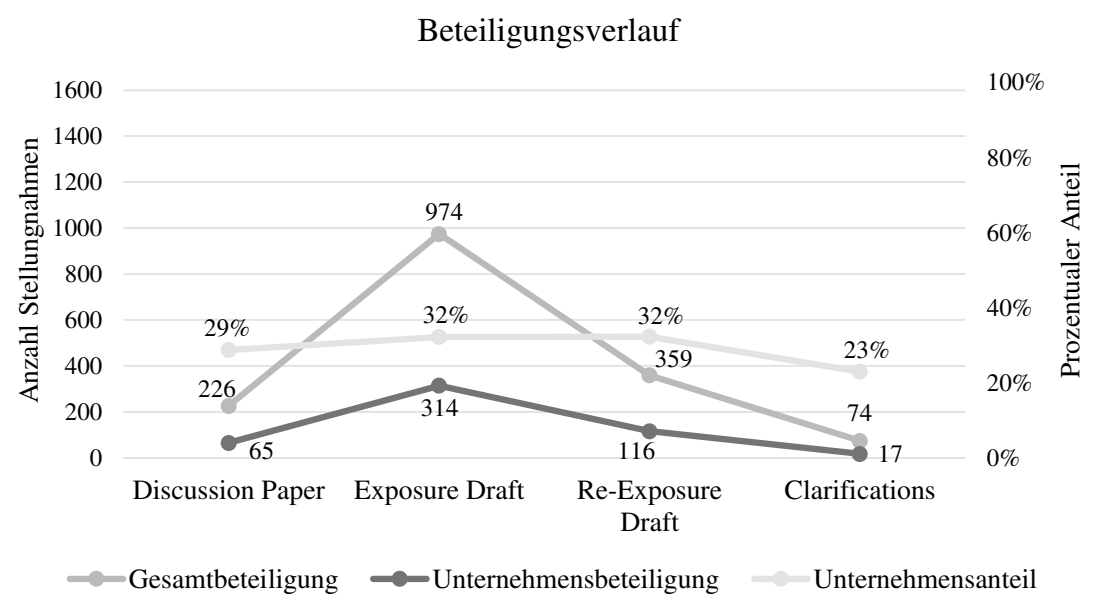

Abbildung 3.1 Beteiligungsverlauf in jeweiliger Kommentierungsphase

Ein möglicher Grund für die am Prozessanfang geringere Beteiligung könnte der zu Beginn des Projekts noch unklare, in der Ferne liegende Anwendungszeitpunkt darstellen, der zunächst keine Notwendigkeit der Auseinandersetzung hervorrief. ${ }^{260}$ Trotz der im Vergleich zum Discussion Paper höheren Beteiligung in den späteren Kommentierungsphasen besteht vom Exposure Draft zum Re-Exposure Draft ein Beteiligungsrückgang, der als Akzeptanz des überarbeiteten Entwurfs gedeutet werden kann. ${ }^{261}$ Die im Vergleich zu den anderen Kommentierungsphasen sehr niedrige Beteiligung im Rahmen der nachträglichen Klarstellung lässt sich in Einklang mit durchgeführten Studien zum Zusammenhang zwischen dem Ausmaß der Beteiligung und der Relevanz des Standards für die Beteiligten mit dem themenspezifischen Fokus des Standardentwurfs erklären. ${ }^{262}$ Der relative Beteiligungsanteil der Unternehmen in den einzelnen Kommentierungsphasen ist hingegen überwiegend konstant bei etwa $30 \%$, was das für die Auswahl der Interessengruppe angenommene kontinuierlich hohe Beteiligungsverhalten bestätigt.

${ }^{260}$ Vgl. Kurz (2009), S. 173.

261 Vgl. Pelger (2012), S. 103; Technical Staff (2012c), Agenda Paper 7 A/160 A, Rz. 6-9.

${ }^{262}$ Vgl. Tandy/Wilburn (1992), S. 54; Larson/Herz (2013), S. 125-128. 
Die Verteilung der 512 untersuchten Stellungnahmen auf die acht Herkunftsregionen zeigt eine - mit $66 \%$ deutliche - Mehrheit der US-amerikanischen Beteiligung (Abbildung 3.2). ${ }^{263}$

\section{Herkunftsverteilung der Stellungnahmen}

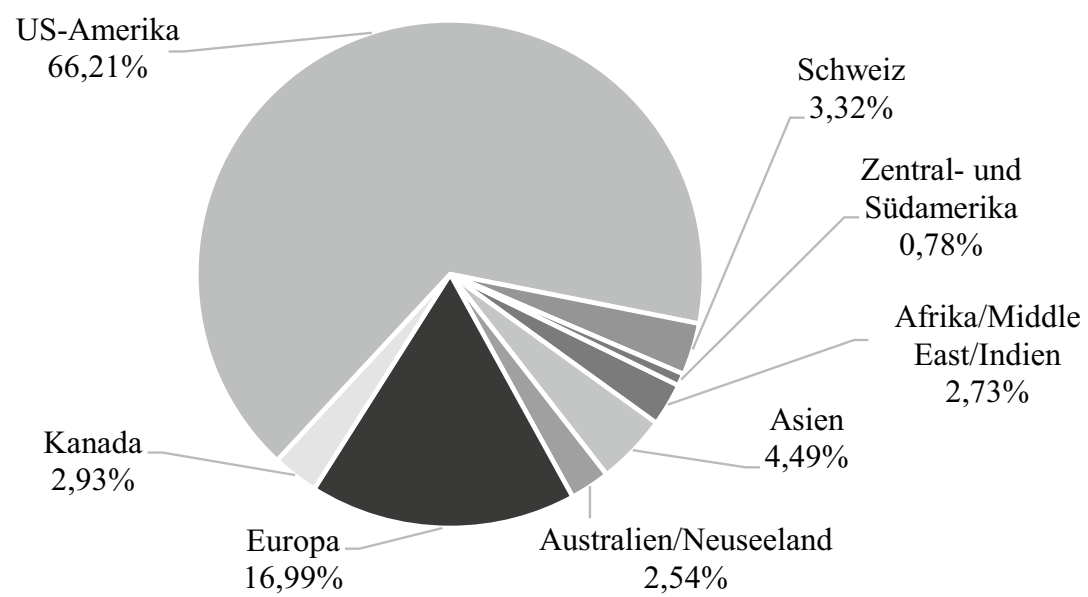

Abbildung 3.2 Herkunftsverteilung der Stellungnahmen

Die hohe Beteiligungsrate US-amerikanischer Unternehmen im Vergleich zu anderen Herkunftsregionen lässt sich durch das regulatorische Umfeld begründen. So ist die Anwendung der US-GAAP für eine viel größere Anzahl an Unternehmen verpflichtend als etwa die Anwendung der IFRS für kapitalmarktorientierte Unternehmen innerhalb der EU. Zudem lässt sich das höhere Beteiligungsverhalten US-amerikanischer Unternehmen auf die Erfahrung mit öffentlichen Standardsetzungsverfahren zurückführen; im Gegensatz hierzu sind die Teilnahmehürden für Unternehmen größer, deren Gesetzgebung auf nationaler Ebene durch staatlich delegierte Verfahren erfolgt und die daher wenig Partizipationserfahrung haben. ${ }^{264}$ Die besonders niedrige Rate von $0,78 \%$ zentral- und südamerikanischer Stellungnahmen sowie der niedrige Anteil afrikanischer und nahöstlicher Stellungnahmen mit 2,73 \% kann neben diesen regulatorischen

263 Vgl. zur Anzahl und zum Anteil der Stellungnahmen nach Herkunft Appendix, IV. 264 Vgl. Orens u. a. (2011), S. 217. 
Bedingungen etwa durch die meist in Entwicklungsländern fehlenden Ressourcen zur Teilnahme sowie die Sprachbarriere ${ }^{265}$ begründet werden. ${ }^{266}$ Trotz der Bestrebung des IASB nach einer geographisch breiten Teilnahme am Standardsetzungsprozess wird die Wahrnehmung, that the IASB is [...] much of a get-together between the USA and Europe“ ${ }^{\text {267 }}$ insofern durch die Ergebnisse der Herkunftsverteilung bestätigt. ${ }^{268}$

Im Hinblick auf die Branchenverteilung der 512 Stellungnahmen ist eine Dominanz der Branche der Fertigungsindustrie festzustellen (Abbildung 3.3) ${ }^{269}$, die mit 48,24 \% fast die Hälfte der untersuchten Stellungnahmen abbildet. Die höhere Beteiligung im Vergleich zu anderen Branchen kann auf die zur Branche der Fertigungsindustrie gehörenden Vielzahl an Industriezweigen und der damit einhergehenden Anzahl sowohl großer als auch kleinerer Unternehmen zurückgeführt werden. Zudem lässt sich die hohe Teilnahme mit der Betroffenheit der Unternehmen dieser Branche aufgrund der anfänglich geplanten Abschaffung der - für diese Branche relevanten - Percentage-of-Completion-Methode begründen. ${ }^{270}$

Das Ausmaß der Standardänderung im Vergleich zur bestehenden Bilanzierungspraxis gilt gleichermaßen für den Beteiligungsgrad der anderen Branchen. ${ }^{271}$ So weist bspw. die Telekommunikationsbranche eine Beteiligungsrate von 10,55 \% auf, die mit den Regelungsänderungen zur Contingent-RevenueCap-Methode in Zusammenhang gebracht werden kann, wohingegen sich Unternehmen der Dienstleistungsbranche, für die sich während des Standardsetzungsprozesses wenig Änderungen andeuteten, mit einem Anteil von 2,54\% nur wenig beteiligten.

Von den 384 Unternehmen haben 38 Unternehmen in allen drei Kommentierungsphasen zum Discussion Paper, Exposure Draft und Re-Exposure Draft teilgenommen, vier dieser Unternehmen beteiligten sich zusätzlich auch in der Phase der nachträglichen Klarstellung. ${ }^{272}$ Die relative Beteiligung von 9,9\% der Unternehmen bestätigt die Ergebnisse von Beteiligungsstudien, wonach eine

\footnotetext{
265 Vgl. Standish (2003), S. 189; Jorissen u. a. (2013), S. 254.

266 Vgl. Larson/Herz (2013), S. 131.

${ }^{267}$ Hoogervorst (2011).

268 Vgl. ebenso etwa Auste (2011), S. 129 f.; Larson/Herz (2013), S. 113-117.

${ }^{269}$ Vgl. zur Anzahl und zum Anteil der Stellungnahmen nach Branche Appendix, V.

270 Vgl. Hüfner/Meyer (2018), S. 169.

${ }^{271} \mathrm{Vgl}$. Elbannan/McKinley (2006), S. 610 f.

${ }^{272}$ Vgl. zur Übersicht der Unternehmen mit Beteiligung in allen Kommentierungsphasen Appendix, VI.
} 
Branchenverteilung der Stellungnahmen

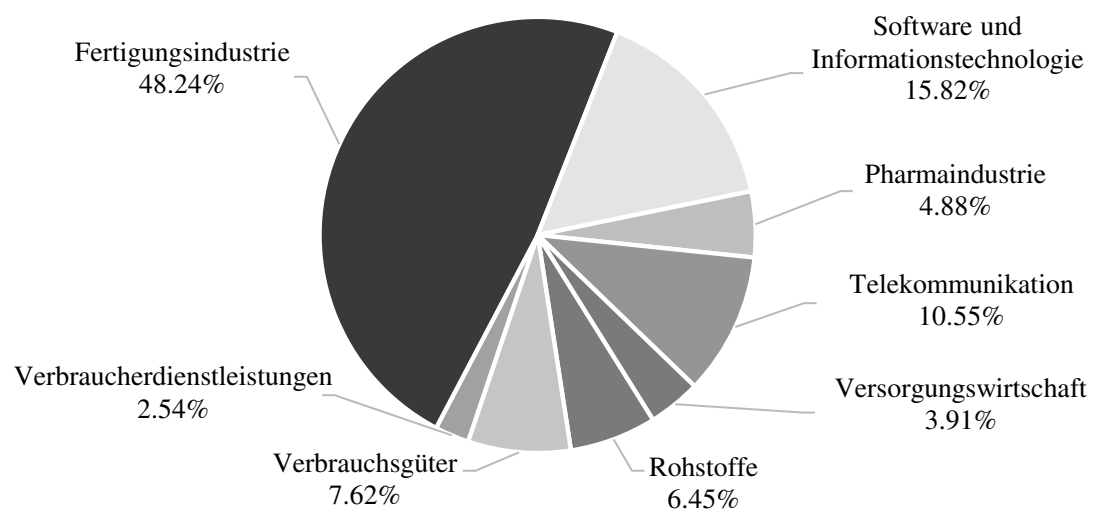

Abbildung 3.3 Branchenverteilung der Stellungnahmen

regelmäßige Teilnahme von den weltweit größten Unternehmen festzustellen ist. ${ }^{273}$ In Einklang mit der Herkunfts- und Branchenverteilung handelt es sich bei der Mehrheit der Unternehmen um US-amerikanische sowie europäische Unternehmen der Fertigungsindustrie, Telekommunikations- und Softwarebranche.

\subsubsection{Inhaltliche Zielrichtung und Rhetorik im Standardsetzungsprozess von IFRS 15}

\subsubsection{Unternehmenseinfluss auf die inhaltliche Zielrichtung}

\subsection{Häufigkeit kommentierter Bilanzierungsalternativen und Kookkurrenz der Umsetzungsmöglichkeiten}

Nach dem Überblick der beteiligten Unternehmen wird im Folgenden zunächst die inhaltliche Zielrichtung der Unternehmen sowie der Standardsetzer ausgewertet, um Erkenntnisse über den wechselseitigen Einfluss der Standardsetzer und der Unternehmen auf das Standardergebnis zu erlangen. Die Auswertung erfolgt auf Basis von Themenübersichten, die die Häufigkeit der kommentierten Bilanzierungsalternativen im Verhältnis zur Unternehmensbeteiligung sowie

${ }^{273}$ Vgl. etwa Larson (1997), S. 189 f. 
die von den Standardsetzern diskutierten Bilanzierungsalternativen (mit * gekennzeichnet) abbilden, ${ }^{274}$ insofern jedoch nicht mit der Zustimmung oder Ablehnung der Unternehmen und Standardsetzer zu der jeweiligen Bilanzierungsalternative gleichzusetzen sind. So lehnten etwa die Standardsetzer die Möglichkeit Leistungsverpflichtungen nicht aufzuteilen $\mathrm{ab}$, diskutierten sie indes trotzdem in der Basis for Conclusions zum Exposure Draft. ${ }^{275}$ Durch die thematische Erfassung wird dem qualitativen Inhalt, d. h. der Bezugnahme auf die konkreten Äußerungen, der Stellungnahmen und Standarddokumente Rechnung getragen; ${ }^{276}$ gleichzeitig sind jedoch quantifizierbare Aussagen über die Themenschwerpunkte und den Verlauf der diskutierten Bilanzierungsalternativen und Umsetzungsmöglichkeiten in den einzelnen Kommentierungsphasen möglich. Die Ergebnisse werden zudem anhand von Auszügen aus den Stellungnahmen und Standardentwürfen beispielhaft veranschaulicht. Die kommentierten Umsetzungsmöglichkeiten werden mithilfe der Kookkurrenz der adressierten Bilanzierungsalternativen, d. h. dem gemeinsamen Auftreten der Kategorien, berücksichtigt, um den Zusammenhang zwischen dem Inhalt und der Form der Standardausgestaltung beurteilen zu können. ${ }^{277}$

\subsection{Anknüpfung an alte Regelungsvorschriften und praxisorientierte Regelungen bei den Ausnahmen vom einzelvertragsbasierten Ansatz}

Im Hinblick auf die untersuchten Bilanzierungsalternativen zu den Ausnahmen vom vertragsbasierten Ansatz (Tabelle 3.9) adressierte in der Kommentierungsphase zum Discussion Paper etwa jedes fünfte Unternehmen die Zusammenfassung von Verträgen.

Obwohl das Discussion Paper die Vertragszusammenfassung als ,a matter not discussed“ behandelte, ${ }^{278}$ nutzten $21,54 \%$ der Unternehmen die Kommentierungsphase, um zu diesem Thema Stellung zu nehmen, was den proaktiven Einfluss von Unternehmen auf die Regelungsausgestaltung verdeutlicht. Die

\footnotetext{
274 Vgl. zur Gesamtübersicht der Häufigkeit kommentierter Bilanzierungsalternativen und Umsetzungsmöglichkeiten in den einzelnen Kommentierungsphasen Appendix, VII.

275 Vgl. ED BC (2010), BC46.

${ }^{276}$ Vgl. zur Beziehung zwischen Kategorie und Ausgangsmaterial Kuckartz (2018), S. 42 f. ${ }^{277}$ In QDAMiner wird die Kookkurrenz mithilfe der Coding-Co-occurrences getestet, wobei die Berechnung für die inhaltliche Zielrichtung jeweils auf Unternehmensebene erfolgte, d. h. mehrfache Kookkurrenzen in einer Unternehmensstellungnahme wurden nur einfach gewertet.

${ }^{278}$ Vgl. DP (2008), 7.27.
} 
Tabelle 3.9 Anteil der Unternehmensstellungnahmen zu den Ausnahmen vom vertragsbasierten Ansatz in den einzelnen Kommentierungsphasen

\begin{tabular}{l|l|l|l|l}
\hline Ausnahmen vertragsbasierter Ansatz & DP & ED & Re-ED & CL \\
\hline $\begin{array}{l}\text { Aufteilung eines Vertrags in mehrere } \\
\text { Einzelverträge }\end{array}$ & - & $14,65 \% *$ & $0,86 \% *$ & - \\
\hline Portfoliobetrachtung & $6,15 \%$ & $2,55 \%$ & $7,76 \% *$ & - \\
\hline Zusammenfassung von Verträgen & $21,54 \% *$ & $24,52 \% *$ & $2,59 \% *$ & - \\
\hline
\end{tabular}

beteiligten Unternehmen waren fast ausschließlich US-amerikanische Unternehmen der Fertigungsindustrie, die sich überwiegend für die Übernahme alter Regelungen aussprachen, ${ }^{279}$ was die Vermutung bekräftigt, dass bei Änderungen eines Rechnungslegungsstandards Unternehmen auf die Beibehaltung bestehender Regelungsvorschriften hinwirken.

„We believe combining contracts [...] is appropriate and the guidance in SOP 81-1 should be retained." (Boeing) ${ }^{280}$

„In addition, we believe there should be a provision allowing the aggregation of a group of E\&C contracts as provided by SOP $81-1 . “\left(\right.$ Kiewit) ${ }^{281}$

Auch das noch vor dem Ende der Kommentierungsfrist erlangte Ergebnis des Staff, die Zusammenfassung von Verträgen an das Prinzip der Preisinterdependenz zu knüpfen, basierte auf den alten Regelungen des SOP 81-1 sowie des IAS 11. ${ }^{282}$ Die Boards begründeten die vorgenommenen Änderungen im Exposure Draft übereinstimmend mit den Staff-Empfehlungen und Begründungen, ohne jeglichen Verweis auf die Stellungnahmen. ${ }^{283}$ Die Anknüpfung an die alten Regelungsvorschriften zur Ausgestaltung der Regelungen zur Vertragszusammenfassung kann angesichts der fehlenden Bezugnahme insofern nicht direkt mit dem Unternehmenseinfluss in Verbindung gebracht werden, wenngleich sie vor dem Hintergrund der zwischen den Standardsetzern und Unternehmen bestehenden

${ }^{279}$ Die Kookkurrenz zwischen Zusammenfassung von Verträgen und Übernahme alter Regelungsvorschriften zeigt 10 von 14 Unternehmen, mithin 71,43\%. Von den 10 Unternehmen sind 7 Unternehmen aus der Fertigungsindustrie mit US-amerikanischer Herkunft (70,00\%).

${ }^{280}$ Boeing (2009), S. 3.

${ }^{281}$ Kiewit (2009), S. 5.

${ }^{282}$ Vgl. Technical Staff (2009b), Agenda Paper 7B/118B, Rz. 7-18.

283 Vgl. ED BC (2010), BC36. 
Einigkeit die konsensfähige Bilanzierungsalternative darstellte. In den Stellungnahmen zum Exposure Draft adressierten dennoch etwa die Hälfte der zu diesem Thema beteiligten Unternehmen die vorgesehenen Regelungen in Bezug auf den Umfang und die Ausgestaltung der Indikatoren oder befürworteten zusätzliche Anwendungshilfen, ${ }^{284}$ wobei die Relevanz des ,,negotiated business intent“ ${ }^{285}$ für Unternehmen als Faktor deutlich wurde.

„The terminology used here should potentially be amended so that it refers to substance of two contracts and that if the goods or services of one contract are linked in terms of delivery and customer usage." (EasyJet) ${ }^{286}$

Die Anforderung der Preisinterdependenz wurde im Re-Exposure Draft und letztlich auch im finalen Standard nicht mehr als notwendiges, sondern als hinreichendes Kriterium neben der Anforderung des gemeinsamen wirtschaftlichen Zwecks festgelegt, ${ }^{287}$ was insofern eine Annäherung an die vorherigen Regelungen des SOP 81-1 darstellte. Die explizite Bezugnahme der Boards auf die „respondents“ in der Basis for Conclusions ${ }^{288}$ und die insoweit übereinstimmende Änderung in Einklang mit den von Unternehmen adressierten Umsetzungsmöglichkeiten sowie der auf 2,59\% gesunkene Anteil an Unternehmen, die die Regelungen im Re-Exposure Draft kommentierten, legt für die Regelungen zur Vertragszusammenfassung einen Unternehmenseinfluss nahe.

Im Zuge des Exposure Drafts normierten die Standardsetzer die Preisinterdependenz nicht nur als Kriterium zur Vertragszusammenfassung, sondern auch als Kriterium zur Aufteilung eines Vertrags in mehrere Einzelverträge. ${ }^{289}$ Die zu diesem Thema beteiligten Unternehmen stellten in der Kommentierungsphase zum Exposure Draft die Notwendigkeit des Regelungsvorschlags hingegen überwiegend in Frage. Dabei wurde insbesondere die Aufteilung eines Vertrags in Einzelverträge im Verhältnis zur Aufteilung von Leistungsverpflichtungen kritisiert.

\footnotetext{
${ }^{284}$ Die Kookkurrenz zwischen Zusammenfassung von Verträgen, Umfang der Guidance und Determiniertheit der Regelungsvorschriften zeigt 38 von 77 Unternehmen, mithin 49,35\%.

285 Intel (2010), S. 5.

${ }^{286}$ EasyJet (2010), S. 2.

${ }^{287}$ Vgl. Re-ED (2011), 17; Re-ED BC (2011), BC51-BC53.

288 Vgl. Re-ED BC (2011), BC53 (auch Zitat).

${ }^{289} \mathrm{Vgl}$. ED (2010), 15.
} 
„However, we question the necessity of this additional step and whether the accounting result would be different in most cases than if an entity simply identified separate performance obligations." (IBM) ${ }^{290}$

„We believe that the two-step process of segmenting a contract and then identifying separate performance obligations is unnecessarily complex and provides little incremental benefit. Accordingly, we recommend that the Boards merge the two-step process into a single one based on the identification of distinct performance obligations." (Lockheed Martin) $)^{291}$

Die Überschneidung beider Kriterien wurde auch vom Staff bei der Auswertung aller Stellungnahmen als Kritikpunkt identifiziert und ein „single step approach“ empfohlen, ${ }^{292}$ was insoweit in Einklang mit den Unternehmensstellungnahmen stand. Die Boards begründeten die Abschaffung der Regelung im Re-Exposure Draft indes zwar ohne Bezug zu den Stellungnahmen, die Begründung weist jedoch eine hohe Übereinstimmung mit den Unternehmenskommentierungen auf.

„In their re-deliberations on the 2010 exposure draft, the boards decided to eliminate the step of segmenting a contract into separate (hypothetical) contracts because that step is unnecessary. The boards noted that the proposed requirement to identify the separate performance obligations in a contract achieves the same result as accounting for the separate components of a contract." (IASB/FASB) 293

Diese Kongruenz sowie die ausgebliebenen Stellungnahmen zu diesem Thema sowohl zum Discussion Paper als auch zum Re-Exposure Draft, die auf die fehlende Relevanz der Bilanzierungsalternative für Unternehmen hindeuten, unterstützen die Annahme, dass die Nicht-Umsetzung der Regelungsvorschriften zur Aufteilung eines Vertrags in mehrere Einzelverträge auch auf die Unternehmensstellungnahmen zurückgeführt werden kann.

Im Gegensatz zur Einzelvertragsaufteilung zeigt die proaktive Kommentierung des Portfolio-Ansatzes die Relevanz dieser Bilanzierungsalternative für Unternehmen, wobei es sich fast ausschließlich um Unternehmen der Telekommunikationsbranche ${ }^{294}$ handelte und insofern auf eine branchenspezifische Bilanzierungsalternative hindeutet. In der Kommentierungsphase zum Discussion

\footnotetext{
290 IBM (2010), S. 5.

${ }^{291}$ Lockheed Martin (2010), S. 2.

292 Vgl. Technical Staff (2011a), Agenda Paper 6B/135B, Rz. 6 f., 12 (auch Zitat, Rz. 7).

293 Re-ED BC (2011), BC49.

${ }^{294}$ Im Discussion Paper waren 3 der 4 Unternehmen und im Exposure Draft 6 der 8 Unternehmen aus der Telekommunikationsbranche $(75,00 \%)$.
} 
Paper als auch zum Exposure Draft befürworteten 6,15 \% bzw. 2,55 \% der Unternehmen eine Portfolio-Betrachtung von Verträgen bzw. Leistungsverpflichtungen, ohne dass eine derartige Bilanzierungsmöglichkeit von den Standardsetzern diskutiert wurde.

„Recognising assets and liabilities using a portfolio basis would be easier to implement and we do believe that the information provided in the financial statements would not be different." (Deutsche Telekom) ${ }^{295}$

„For industries similar to ours, we believe that an option to use a portfolio based approach for homogeneous transactions would make this standard easier to implement while not materially changing the financial statements." (Sprint Nextel) ${ }^{296}$

Die Portfolio-Option wurde vom Staff in einer auf dem Feedback der Beteiligten basierenden Zusammenfassung der Standardauswirkungen für Telekommunikationsunternehmen als Möglichkeit empfohlen, die Bedenken dieser Unternehmen ohne Überarbeitung des Umsatzerfassungsmodells $\mathrm{zu}$ adressieren. ${ }^{297}$ In Einklang hierzu führten die Boards im Re-Exposure Draft die Portfolio-Option als ,practical expedient“ ein (IFRS 15.4) und begründeten dies unter expliziter Bezugnahme auf die ,entities“, 298 was einen Unternehmenseinfluss bestätigt. Der Anstieg der Unternehmenskommentierungen zum Portfolio-Ansatz auf 7,76 \% in der Kommentierungsphase zum Re-Exposure Draft ist vor diesem Hintergrund überraschend, insbesondere aufgrund der von Unternehmen nunmehr geäußerten Bedenken.

„Although we appreciate the Board's clarification on the application of a portfolio approach, we are still firmly convinced that applying this practical expedient would not be feasible." (Deutsche Telekom) ${ }^{299}$

„While the portfolio approach could provide some relief (while also introducing another element of aggregation risk which could reduce comparability) from the cost and complexity of implementation, this would still be a significant task for many entities both at implementation and prospectively on an ongoing basis." (Sprint Nextel) $)^{300}$

\footnotetext{
295 Deutsche Telekom (2009), S. 6.

296 Sprint Nextel (2010), S. 4 f.

297 Vgl. Technical Staff (2011b), Agenda Paper 4B/147B, Rz. 18 (c).

${ }^{298}$ Vgl. Re-ED (2011), 6 (auch Zitat); Re-ED BC (2011), BC341 (auch Zitat).

299 Deutsche Telekom, S. 2.

${ }^{300}$ Sprint Nextel (2012), S. 4.
} 
Diese Widersprüchlichkeit kann zumindest teilweise auf die (fehlende) Umsetzung der Bilanzierungsalternativen zur Residualwertmethode und ContingentRevenue-Cap-Methode zurückgeführt werden, ${ }^{301}$ sie zeigt jedoch gleichzeitig die Grenzen des Unternehmenseinflusses auf. So behielten die Boards den PortfolioAnsatz auch im finalen Standard bei und betonten den branchenspezifischen Nutzen, besonders für die Telekommunikationsbranche. ${ }^{302}$ Diese Kontinuität der Standardsetzer sowie die Ausgestaltung als allgemeine Regelung anstatt als industriespezifische Ausnahmeregelung verdeutlicht den Kompromiss zwischen der Berücksichtigung von Stellungnahmen der Unternehmen und der Umsetzung der von den Standardsetzern angestrebten Standardsetzungsziele.

\subsection{Anwendungsorientierte Konkretisierung der Regelungen zur Aufteilung von Leistungsverpflichtungen}

Die Aufteilung eines Vertrags in einzelne Leistungsverpflichtungen wurde im Discussion Paper zunächst an den Zeitpunkt der Verpflichtungserfüllung geknüpft. Eine Separierung war demnach erforderlich, wenn der Kunde die vereinbarten Güter oder Dienstleistungen zu unterschiedlichen Zeitpunkten erhält. ${ }^{303}$

Tabelle 3.10 Anteil der Unternehmensstellungnahmen zur Aufteilung von Leistungsverpflichtungen in den einzelnen Kommentierungsphasen

\begin{tabular}{l|l|l|r|l}
\hline Aufteilung Leistungsverpflichtungen & DP & ED & Re-ED & CL \\
\hline Anknüpfung an den Erfüllungszeitpunkt & $36,92 \% *$ & $1,27 \% *$ & - & - \\
\hline Distinct-Kriterium & - & $23,57 \% *$ & $22,41 \% *$ & $94,12 \% *$ \\
\hline Interdependenz & $9,23 \%$ & $26,11 \% *$ & $7,76 \% *$ & $17,65 \% *$ \\
\hline Gewinnmarge & $10,77 \%$ & $24,20 \% *$ & $-*$ & - \\
\hline Einzelveräußerung & $33,85 \% *$ & $4,46 \% *$ & $1,72 \% *$ & - \\
\hline Risiken & - & $24,84 \% *$ & $-*$ & \\
\hline Vertragsübergreifende Beurteilung & $36,92 \%$ & $36,94 \% *$ & $0,86 \%$ & - \\
\hline
\end{tabular}

Die Anknüpfung an den Erfüllungszeitpunkt wurde im Hinblick auf die Bilanzierungsalternativen zur Aufteilung von Leistungsverpflichtungen (Tabelle 3.10) im Discussion Paper von 36,92 \% der Unternehmen kommentiert, wobei viele

${ }^{301}$ Die Kookkurrenz zwischen Portfoliobetrachtung und Residualwertmethode sowie Contingent-Revenue-Cap-Methode zeigt 4 von 9 Unternehmen, mithin 44,44\%.

302 IFRS 15.BC69 f.

${ }^{303}$ Vgl. DP (2008), 3.24. 
Unternehmen Bedenken äußerten und bspw. die Unbestimmtheit des Kriteriums bzw. den Umfang der Guidance kritisierten. ${ }^{304}$

„Furthermore it seems to us that the definition for separating performance obligation is not clear enough." (Swatch Group) $)^{305}$

„The Discussion Paper does not address how performance obligations should be aggregated or disaggregated, other than to state that performance obligations that are transferred to customers at different times should be separated. We believe additional guidance is necessary.“ (Intel) $)^{306}$

Als Alternativvorschlag ist die von ebenfalls $36,92 \%$ der Unternehmen befürwortete vertragsübergreifende Beurteilung $\mathrm{zu}$ werten, wonach der gesamte Vertrag als wirtschaftliche Einheit zu betrachten ist. Bei den Unternehmen handelte es sich überwiegend um Unternehmen aus der Fertigungsindustrie sowie mit USamerikanischer Herkunft, ${ }^{307}$ die hierfür die Übernahme alter branchenspezifischer Regelungen vorschlugen. ${ }^{308}$

\begin{abstract}
„We believe the inclusion of elements of SOP 81-1 in the proposed model would better fit the economic rationale for our contracts, versus requiring us to account for contract elements based on timing of delivery. We thus recommend the Boards consider the contract as the appropriate unit of account and include the separation criteria under SOP 81-1 in the proposed model, adding further guiding principles to promote consistency in application." (Northrop Grumman) ${ }^{309}$

„Thus, we recommend that the Discussion Paper be modified to presume that the contract is the basic unit of account and to provide guidance on when it is appropriate to overcome this presumption [...]. This guidance could borrow from the concepts in SOP 81-1 and IAS 11.“ (General Dynamics) ${ }^{310}$
\end{abstract}

\footnotetext{
${ }^{304}$ Die Kookkurrenz zwischen Anknüpfung an den Erfüllungszeitpunkt und Umfang der Guidance zeigt 12 von 26 Unternehmen, mithin 46,15\%.

305 Swatch Group (2009), S. 3.

306 Intel (2009), S. 3.

307 Von den 24 Unternehmen sind 17 Unternehmen aus der Fertigungsindustrie $(70,83 \%)$ und 14 Unternehmen hiervon sind US-amerikanischer Herkunft (58,33\%).

${ }^{308}$ Die Kookkurrenz zwischen Vertragsübergreifender Ansatz und Übernahme alter Regelungen zeigt 11 von 24 Unternehmen, mithin 45,83\%.

${ }^{309}$ Northrop Grumman (2009), Attachment, S. 4.

${ }^{310}$ General Dynamics (2009), S. 3.
} 
In Zusammenhang mit der vertragsübergreifenden Beurteilung bezogen sich $10,77 \%$ der Unternehmen auf die Gewinnmarge des Vertrags. ${ }^{311}$ Hierbei handelte es sich ausschließlich um US-amerikanische Unternehmen aus der Fertigungsindustrie, die eine Separierung auf Basis des Erfüllungszeitpunkts aufgrund vertragsübergreifender Margen kritisierten.

„We generally negotiate contracts as a single project with an overall profit margin [...]. In such circumstances, the entire contract should represent a single performance obligation unless the economics of the contract clearly indicate that there are components that should be accounted for separately [...].“ (Northrop Grumman) ${ }^{312}$

Für die Aufteilung von Leistungsverpflichtungen verwiesen 9,23\% der wiederum fast ausschließlich aus der Fertigungsindustrie stammenden Unternehmen zudem auf die Interdependenzen bzw. funktionale Abhängigkeit der einzelnen Leistungsverpflichtungen. ${ }^{313}$

„[A]pplying the guidance under the proposed model and unnaturally disaggregating performance obligations could be interpreted to result in immediate loss recognition with respect to that apparent onerous element. We believe this is counter to the way the overall contract is managed and, more importantly, counter to the overall economics of the transaction. On many long-term construction / production-type contracts, the various tasks are deeply interrelated." (Raytheon) $)^{314}$

Das Vorliegen unterschiedlicher „profit margins“ sowie von ,,interrelated or interdependent" Vertragskomponenten wurde von den Standardsetzern im Discussion Paper nicht adressiert. Beide Anforderungen galten jedoch nach den für die Fertigungsindustrie relevanten Standards SOP 81-1 bzw. IAS 11 als Kriterien für die Zusammenfassung bzw. Separierung von Verträgen, ${ }^{315}$ was wiederum zeigt, dass Unternehmen bei Standardüberarbeitungen zunächst an der etablierten Bilanzierungspraxis festhalten.

Für die Abgrenzung von Leistungsverpflichtungen bezogen sich 33,85\% der Unternehmen zudem auf die Möglichkeit der Einzelveräußerung der Güter und Dienstleistungen, die von den Standardsetzern im Discussion Paper als „evidence" der Separierbarkeit, nicht jedoch als maßgebliches Kriterium angeführt

${ }^{311}$ Die Kookkurrenz zwischen Gewinnmarge und Vertragsübergreifender Ansatz zeigt 7 von 7 Unternehmen, mithin $100 \%$.

312 Northrop Grumman (2009), S. 3.

${ }^{313}$ Von den 6 Unternehmen sind 5 Unternehmen aus der Fertigungsindustrie $(83,33 \%)$.

${ }^{314}$ Raytheon (2009), S. 3.

315 Vgl. SOP 81-1.35-1.41; IAS 11.4, 8-10. 
wurde $^{316}$. Bei der Hälfte der Unternehmen handelte es sich um Unternehmen der Software- und Telekommunikationsbranche, ${ }^{317}$ die den Einzelveräußerungswert für den Kunden als (zusätzliche) Anforderung für die Aufteilung von Leistungsverpflichtungen forcierten ${ }^{318}$.

„The company proposes that the definition of a performance obligation [...] be amended to include the concept that the performance obligation must have stand-alone customer value as a good or service." (IBM) ${ }^{319}$

„However, some additional criteria should be met when identifying a performance obligation. Performance obligations should be determined with reference to whether the customer would purchase the asset on a standalone basis in normal circumstances." (Telefónica) ${ }^{320}$

Im Gegensatz zu den Unternehmen der Fertigungsindustrie verwiesen die Unternehmen der Software- und Telekommunikationsbranche überwiegend nicht explizit auf die vorherigen Regelungsvorschriften, der ,value to the customer on a standalone basis" stellte jedoch in den für diese Branche einschlägigen Standards EITF 00-2 $1^{321}$ bzw. ASC 605-25 322 das Kriterium zur Abgrenzung dar. Dies bestätigt wiederum die Erkenntnis, dass Unternehmen bei Standardänderungen die geplanten Regelungen zugunsten bestehender Vorschriften zu beeinflussen versuchen.

Auf die Anknüpfung an die jeweils branchenspezifischen Regelungen in den Unternehmensstellungsnahmen wurde sowohl vom Staff in der Comment-LetterAuswertung $^{323}$ als auch von den Standardsetzern in der Basis for Conclusions zum Exposure Draft explizit Bezug genommen und die Einführung des DistinctKriteriums sowie der konkretisierenden Anforderungen in Zusammenhang mit den Ergebnissen der Stellungnahmen begründet. ${ }^{324}$

\footnotetext{
316 Vgl. DP (2008), 3.11, 3.21, 4.28 (auch Zitat, 4.28).

${ }^{317}$ Von den 22 Unternehmen sind 6 Unternehmen aus der Telekommunikations- und 5 Unternehmen aus der Softwarebranche $(50,00 \%)$.

318 Die Kookkurrenz zwischen Einzelveräußerung und Umfang der Guidance zeigt 4 von 6 Unternehmen der Telekommunikationsbranche und 4 von 5 Unternehmen der Softwarebranche, mithin $72,73 \%$.

319 IBM (2009), S. 3.

320 Telefónica (2009), S. 8.

321 Vgl. EITF 00-21.9 (auch Zitat).

${ }^{322} \mathrm{Vgl}$. ASC 605-25-25-5.

${ }^{323}$ Vgl. Technical Staff (2009a), Agenda Paper 14 A/119 A, Rz. 40-46.

${ }^{324}$ Vgl. ED BC (2010), BC45-BC49.
} 
„Representatives from the construction industry preferred to account for all the promised goods or services in a contract as a single performance obligation unless a part of the contract is regularly sold separately. [...]

In contrast, representatives from other industries (for example, the technology industry) preferred to account for an individual good or service as a separate performance obligation even if it is not sold separately. [...]

Consequently, when considering how entities across various industries should identify separate performance obligations, [...] the boards decided that an entity should account for a promise of a good or service as a separate performance obligation only if that good or service is distinct." (IASB/FASB) $)^{325}$

Die Konkretisierung durch die Anforderungen der ,distinct function“ sowie der „distinct profit margin“ stand dabei in Einklang mit den Unternehmenskommentierungen zum Discussion Paper und insofern mit den vorherigen Regelungen. Dies zeigt den Einfluss der Unternehmen auf die Regelungsausgestaltung, gleichzeitig auch die Grenzen einer konzeptionellen Neuausrichtung auf.

Der Rückgang der Unternehmensstellungnahmen im Exposure Draft zur Anknüpfung an den Erfüllungszeitpunkt und zur Einzelveräußerung sowie die demgegenüber gestiegene Beteiligung zu den anderen Bilanzierungsalternativen verdeutlicht die Rekursivität des Standardsetzungsprozesses, da die Unternehmen auf die Änderungen der Standardsetzer eingehen und diese wiederum konkretisieren, gleichzeitig jedoch in der vorherigen Regelungspraxis verhaftet sind.

„Telefónica supports the separation of performance obligations, but we consider that the notion of "distinct" is not clear enough. [...] Further, we believe that determining how to unbundle performance obligations [...] should not include deliverables that do not have value to the customer on a standalone basis [...].“ (Telefónica) ${ }^{326}$

In Bezug auf das Distinct-Kriterium forderten etwa die Hälfte der 23,57 \% beteiligten Unternehmen zusätzliche Anwendungsleitlinien, ${ }^{327}$ was erneut die Akzeptanz des Kriteriums, zugleich jedoch auch die Konkretisierungsbestrebung zugunsten einer anwendungsorientierten Regelungsausgestaltung, widerspiegelt.

\footnotetext{
325 ED BC (2010), BC46.

326 Telefónica (2010), S. 3.

${ }^{327}$ Die Kookkurrenz zwischen Distinct-Kriterium und Umfang der Guidance zeigt 36 von 73 Unternehmen, mithin 49,32\%.
} 
„We believe that the concept of "distinct" is a reasonable basis for identifying separate performance obligations. However, we request additional guidance and examples to clarify the application of this concept in practice." (Nokia) ${ }^{328}$

„We believe further clarification is needed for the proposed principle of determining when a good or service is distinct. [...] We believe it would be helpful if the Board developed more complex examples (perhaps as an extension to existing example 10) in the proposed guidance." (XenoPort) ${ }^{329}$

Etwa jedes vierte Unternehmen bezog sich konkret auf die Anforderung der eigenständigen Gewinnmarge sowie der eigenständigen Funktion. Im Hinblick auf die Verbindlichkeit der Anforderung der Gewinnmarge sowie den Umfang der Anwendungsleitlinien äußerten sich etwa $30 \%$ der Unternehmen kritisch, wobei es sich hierbei überwiegend um Unternehmen der Softwarebranche handelte. ${ }^{330}$

„Distinct profit margin should be an indicator rather than specific criteria.“ (Dell) ${ }^{331}$

„We propose that the Boards please clarify the distinct profit margin standard and consider that a good or service can have a distinct profit margin if the entity has a reasonable basis to estimate a profit margin." (Symantec) $)^{332}$

„We support the idea of identifying the individual performance obligations within a contract and separately accounting for each obligation, but we would seek additional guidance on the application of the distinct profit margin guidance in determining contract performance obligations." (IHS Inc.) ${ }^{333}$

Für das Vorliegen einer separaten Gewinnmarge wurde von den Standardsetzern in der Basis for Conclusions zum Exposure Draft auf die separaten Risiken der Güter oder Dienstleistungen verwiesen und diese für die Fertigungsindustrie exemplarisch erläutert. ${ }^{334}$ Die Beteiligung von $24,84 \%$ erfolgte in Einklang hierzu fast ausschließlich von Unternehmen der Fertigungsindustrie,

\footnotetext{
${ }^{328}$ Nokia (2010), S. 2.

${ }^{329}$ Xenoport (2010), S. 1.

${ }^{330}$ Die Kookkurrenz zwischen Gewinnmarge und Determiniertheit der Regelungsvorschriften sowie Umfang der Guidance zeigt 23 von 76 Unternehmen, mithin 30,26 \%, wobei 14 von 23 Unternehmen aus der Softwarebranche stammen $(60,87 \%)$.

331 Dell (2010), S. 8.

332 Symantec (2010), S. 2.

333 IHS Inc. (2010), Appendix, S. 1.

334 Vgl. ED BC (2010), BC56-BC59.
} 
die eine Aufteilung von Leistungsverpflichtungen aufgrund vertragsübergreifender Risiken kritisierten bzw. vor diesem Hintergrund die Risikobeurteilung als Konkretisierung des Distinct-Kriteriums im Standard befürworteten. ${ }^{335}$

\begin{abstract}
„Specifically, we request that the Boards recognize that in most cases, all construction activities for a given project [...] have overall risks which are inseparable. Therefore, construction companies like ourselves lack a basis for determining the price at which it would sell the components of a contract separately. Characteristics of distinct profit margin will not be met (in most cases) and hence there is typically no more than a single performance obligation for most construction contracts." (MYR Group) ${ }^{336}$

„We appreciate that the Board has acknowledged in the application guidance in Paragraphs BC56 - BC59 that, in many instances, it does not make sense to separate long-term contracts into multiple performance obligations due to significant overarching contract management services and pervasive risks involved in the production of highly complex deliverables. We believe that this concept should have more prominence in the proposed standard, supplementing the guidance provided in paragraphs 23 (a) and (b) for determining whether a good or service, or a bundle of goods or services, is distinct." (Bombardier) ${ }^{337}$
\end{abstract}

Auch die Anforderung der separaten Funktion wurde überwiegend von Unternehmen der Fertigungsindustrie sowie von Unternehmen der Softwarebranche kommentiert und durch verschiedene Indikatoren, etwa der Kundenspezifizierung und der Integrationsleistung, konkretisiert. ${ }^{338}$

„We believe the Boards should consider the following indicators when determining whether a contract represents a single performance obligation: The product or services delivered are highly customized, integrated, customer specific and delivered over an extended period.“ (Lockheed Martin) 339

\footnotetext{
335 Von 78 Unternehmen sind 71 Unternehmen aus der Fertigungsindustrie (91,03\%). Die Kookkurrenz zwischen Risiken und Vertragsübergreifender Ansatz zeigt 57 von 71 Unternehmen, mithin 80,28 \%. Die Kookkurrenz zwischen Risiken und der Determiniertheit der Regelungsvorschriften sowie Umfang der Guidance zeigt 7 von 71 Unternehmen, mithin $9,86 \%$.

336 MYR Group (2010), S. 2.

337 Bombardier (2010), S. 4.

338 Von den 82 Unternehmen sind 67 aus der Fertigungsindustrie $(81,71 \%)$ und 10 Unternehmen aus der Softwarebranche $(12,20 \%)$.

${ }^{339}$ Lockheed Martin (2010), S. 3.
} 
„[F]or software arrangements the topics of functional interdependence (e.g. essentiality and services for significant production, modification, or customization of the software) should be covered [...].“(SAP) ${ }^{340}$

Mit 36,94 \% befürworteten indes auch im Exposure Draft ein im Vergleich zum Discussion Paper fast gleicher Anteil an Unternehmen einen vertragsübergreifenden Ansatz. Hiervon handelte es sich bei 40,52 \% um annähernd identische Stellungnahmen von ausschließlich US-amerikanischen Unternehmen der Fertigungsindustrie, ${ }^{341}$ was auf Absprachen zwischen diesen Unternehmen hindeutet. In Zusammenhang mit dem vertragsübergreifenden Ansatz forderten Unternehmen sowohl die Übernahme branchenspezifischer Regelungen als auch eine Klarstellung der vorgeschlagenen Kriterien zugunsten einer gesamtvertraglichen Bilanzierung. ${ }^{342}$

„We propose you keep the guidance provided in SOP 81-1, allowing long term construction contracts to be viewed as a single distinct obligation." (National Oilwell (Varco) $)^{343}$

„We believe that the standard should allow for the determination of a single performance obligation as a possible outcome when contract activities are performed in an overlapping, concurrent and/or highly interrelated manner and the pricing of the related activities is not distinct or readily determinable in the market." (ST Marine) $)^{344}$

„We suggest that the Board consider amending the proposed principles such that when an entity negotiates a long-term contract and prices as a single large profit margin for the contract as a whole, it is not necessary to apply the proposed recognition and measurement requirements to each performance obligation separately [...].“ (MWH Global) $^{345}$

\footnotetext{
340 SAP (2010), S. A2.

${ }^{341}$ Von den 116 Unternehmen haben 47 Unternehmen eine fast identische Stellungnahme abgegeben, mithin 40,52 \%. Die Kookkurrenz zwischen Vertragsübergreifender Ansatz, Risiken und Interdependenz zeigt 49 Unternehmen, wovon 2 Stellungnahmen jedoch von den übrigen abweichen.

342 Die Kookkurrenz zwischen Vertragsübergreifender Ansatz und Industriespezifische Ausnahmeregelung bzw. Übernahme alter Regelungen zeigt 47 von 116 Unternehmen, mithin 40,52 \%. Die Kookkurrenz zwischen Vertragsübergreifender Ansatz und Umfang der Guidance bzw. Determiniertheit der Regelungsvorschriften zeigt 16 von 116 Unternehmen, mithin $13,79 \%$.

${ }^{343}$ National Oilwell Varco (2010), S. 3.

${ }^{344}$ ST Marine (2010), S. 2.

${ }^{345}$ MWH Global (2010), S. 4.
} 
Die Kommentierungen zum Exposure Draft veranlassten den Staff und die Standardsetzer zur Überarbeitung einzelner Regelungsvorschriften, insbesondere auch dem Distinct-Kriterium, und der Veröffentlichung eines weiteren Standardentwurfs.

„The staff was persuaded by many of the responses in the comment letters that the criterion proposed in the exposure draft that a good or service is distinct if "the entity, or another entity, sells an identical or similar good or service separately" was too rigid and could inadvertently deem some goods or services to be distinct even though those goods or services might not have a distinct function or distinct risks. Instead, the staff would prefer entities to judge whether the goods or services they promise to their customers possess those attributes." (Staff) 346

„Consequently, some of those respondents suggested that the boards might need to develop industry-specific guidance or create industry-specific exceptions to the general principles. The boards addressed those concerns during the re-deliberations of the proposals in the 2010 exposure draft. [...] In some cases, the changes have resulted in revised requirements that align more closely with existing requirements or current practice than did the proposals in the 2010 exposure draft.

[...] The boards decided unanimously that it was appropriate to go beyond established due process and re-expose their revised revenue proposals because of the importance of the revenue number to all entities and the desire to avoid unintended consequences in the recognition of revenue for specific contracts or industries." (IASB/FASB) ${ }^{347}$

Die Änderungen des Distinct-Kriteriums, etwa in Form der Bilanzierung eines Leistungsbündels im Fall bestehender Interdependenzen oder Modifikationen, erfolgten dabei explizit auf Basis und in Einklang mit den Unternehmenskommentierungen. ${ }^{348}$

„The boards developed the criterion specified in paragraph 29(a) using feedback on the 2010 exposure draft and suggestions from respondents (especially respondents from the construction and manufacturing industries) that the standard should include some of the discussion in the 2010 exposure draft's Basis for Conclusions on distinct profit margins." (IASB/FASB) $)^{349}$

\footnotetext{
346 Technical Staff (2011c), Agenda Paper 6 C/135 C, Rz. 45.

${ }^{347}$ Re-ED BC (2011), BC12-BC14.

348 Vgl. Re-ED BC (2011), BC67-BC81.

${ }^{349}$ Re-ED BC (2011), BC79.
} 
Die im Re-Exposure Draft gesunkene bzw. fehlende Kommentierung einzelner Bilanzierungsalternativen, wie etwa der Gewinnmarge oder dem vertragsübergreifenden Ansatz, kann daher als Akzeptanz der Regelungsausgestaltung gewertet werden. Auch der insgesamt deutliche Rückgang der Unternehmensbeteiligung der Fertigungsindustrie zu den Kriterien zur Aufteilung von Leistungsverpflichtungen bestätigt dieses Ergebnis. ${ }^{350}$ Die beteiligten Unternehmen bezogen sich insbesondere auf das Distinct-Kriterium und die Anforderung der Interdependenz. So forderten Unternehmen der Fertigungsindustrie und Softwarebranche mehrheitlich umfangreichere Anwendungsleitlinien zur Beurteilung eines Leistungsbündels. ${ }^{351}$

„In order to avoid misapplication of the guidance as intended by the Boards, we recommend the Boards provide indicators in paragraph 29(a) to help companies determine whether the goods or services in a bundle are "highly interrelated"."(Boeing) $)^{352}$

„We would ask the Boards to consider including the indicators/criteria set forth in ASC 985 or to include additional implementation guidance that would help companies understand when goods and services are deemed to be highly interrelated.“ $(\text { Cisco })^{353}$

23,08 \% der Stellungnahmen zum Distinct-Kriterium stammten von Unternehmen aus der Rohstoffbranche und Versorgungswirtschaft, die eine Klarstellung der Regelung zur Erfassung einer Reihe eigenständiger Güter oder Dienstleistungen befürworteten. ${ }^{354}$

„We believe that additional guidance is necessary to clarify whether, for example, a contract to supply oil or gas over a period of time is a single performance obligation satisfied over time, or a series of performance obligations, as described further on pages 8 and 9 of this letter." $(\mathrm{BP})^{355}$

„Therefore, we respectively request that the Boards provide additional implementation guidance and an example of how this guidance should be applied to a contract

${ }^{350}$ Von den 33 Unternehmen sind 9 Unternehmen aus der Fertigungsindustrie $(27,27 \%)$.

${ }^{351}$ Die Kookkurrenz von Interdependenz und Umfang der Guidance zeigt 5 von 7 Unternehmen, mithin $71,43 \%$.

352 Boeing (2012), S. 3.

${ }^{353}$ Cisco (2012), S. 3.

354 Von den 26 Unternehmen sind 6 Unternehmen aus der Rohstoff- und Versorgungswirtschaftsbranche $(23,08 \%)$.

${ }^{355}$ BP (2012), S. 2. 
involving repetitive deliveries of the same good or service where the benefits associated with those goods and services is instantaneously controlled and the consumed upon delivery, in order to confirm our interpretation." (NextEra Energy) ${ }^{356}$

In Einklang hierzu wurde im Zuge der erstmaligen Veröffentlichung von IFRS 15 die wechselseitige Beziehung zwischen Gütern und Dienstleistungen als Indikator zur Beurteilung der Separierbarkeit (IFRS 15.29) aufgenommen sowie die Regelung zur „series of distinct goods and services“ klargestellt (IFRS 15.22 (b)).

„The boards decided to include this notion as part of the definition of a performance obligation to simplify the application of the model and to promote consistency in the identification of performance obligations in circumstances in which the entity provides the same good or service consecutively over a period of time (for example, a repetitive service arrangement).“(IASB/FASB $)^{357}$

Obwohl diese Änderungen ohne Bezugnahme auf die Stellungnahmen erfolgten, wird deutlich, dass die Änderungen der Anwendbarkeit der Regelungen dienten und mit den Stellungnahmen übereinstimmten. Die Entwicklung des Distinct-Kriteriums zeigt dabei nicht nur die Rekursivität des Standardsetzungsprozesses, sondern vor allem den diskursiven Erfolg der Kommentierungen in Form der erfolgreichen Etablierung bzw. Prägung der Regelungsinhalte und der Ausgestaltungsform.

Gleichwohl kam es nach der erstmaligen Veröffentlichung von IFRS 15 zu zahlreichen Anwendungsfragen, die das IASB zur Überarbeitung sowie Ergänzung der Beispiele in einer weiteren Standardsetzungsphase veranlassten. ${ }^{358}$ In der Phase der nachträglichen Klarstellung wurden im Hinblick auf die untersuchten Bilanzierungsalternativen von fast allen beteiligten Unternehmen das Distinct-Kriterium sowie vereinzelt zusätzlich die Anforderung der Interdependenz kommentiert. Dies steht in Einklang mit den vom IASB überarbeiteten und zur Stellungnahme aufgeforderten Themen und zeigt insoweit, dass Unternehmen die noch im Re-Exposure Draft hervorgebrachten Alternativvorschläge hinsichtlich anderer untersuchter Bilanzierungsthemen, wie bspw. die von Telekommunikationsunternehmen geforderte Contingent-Revenue-CapMethode, nicht erneut forcierten. Gleichzeitig forderten ein überwiegender Anteil der Unternehmen weitreichendere Anwendungsleitlinien bzw. Klarstellungen zum

\footnotetext{
${ }^{356}$ NextEra Energy (2012), S. 4.

357 IFRS 15.BC113.

358 Vgl. ED Clarifications (2015), BC8-BC24.
} 
Distinct-Kriterium und diskutierten die Übernahme der vom FASB angestrebten Ausnahmeregelung zur Bilanzierung von Versandtätigkeiten. ${ }^{359}$

\begin{abstract}
„We do not agree with the IASB's approach to clarify the application of the concept of 'distinct' by only amending the Illustrative Examples. It is our view that amendments to the Standard are required to clarify the requirements of identifying performance obligations.“ (MTN Group) $)^{360}$

„The FASB has proposed an exemption relating to the accounting for shipping and handling activities that occur after the customer has obtained control of the goods. We consider that the IASB should propose the same exemption to address these issues in order to retain the benefits achieved by the converged standards." (Rio Tinto) ${ }^{361}$
\end{abstract}

Während das IASB den Standardtext zum Distinct-Kriterium im finalen Standard in Einklang mit dem FASB anpasste, lehnte es die Einführung eines Wahlrechts bzw. die Umsetzung einer Ausnahmeregelung aufgrund des angestrebten Standardsetzungsziels eines vertragsübergreifenden Umsatzerfassungsmodels ab. ${ }^{362}$ Dies legt die Vermutung nahe, dass die nachträgliche Klarstellungsphase keine Basis für grundlegende Standardänderungen darstellt und das IASB in Bezug auf einzelfallspezifische Bilanzierungsprobleme an einer konzeptionell konsistenten Standardsetzung gegenüber einer praxisorientiert konvergenten Standardsetzung festhält.

\title{
3.2.2.1.4 Konzeptionell konsistente Ausgestaltung der Regelungen zur Aufteilung des Transaktionspreises unter Gewährung praxisgerechter Ermessensspielräume
}

Die Aufteilung des Transaktionspreises auf eigenständige Leistungsverpflichtungen wurde im Discussion Paper an die relativen (geschätzten) Einzelveräußerungspreise der Güter oder Dienstleistungen geknüpft und als Methoden zur Schätzung der Adjusted-Market-Assessment-Ansatz und der Expected-CostPlus-A-Margin-Ansatz als mögliche, jedoch nicht vorgeschriebene Methoden genannt. ${ }^{363}$

${ }^{359}$ Die Kookkurrenz von Distinct-Kriterium und Umfang der Guidance zeigt 9 von 16 Unternehmen, mithin 56,25 \%, und die Kookkurrenz von Distinct-Kriterium und Determiniertheit der Regelungsvorschriften zeigt 11 von 16 Unternehmen, mithin $68.75 \%$, von denen 6 Unternehmen die Übernahme einer Ausnahmeregelung befürworteten.

${ }^{360}$ MTN Group (2015), S. 2.

${ }^{361}$ Rio Tinto (2015), S. 2.

362 IFRS 15.BC116U.

${ }^{363}$ DP (2008), 5.45-5.48. 
Tabelle 3.11 Anteil der Unternehmensstellungnahmen zur Aufteilung des Transaktionspreises in den einzelnen Kommentierungsphasen

\begin{tabular}{l|l|l|l|l}
\hline Aufteilung Transaktionspreis & DP & ED & Re-ED & CL \\
\hline Alternativer Bewertungsmaßstab & $16,92 \%$ & $2,87 \%$ & - & - \\
\hline Contingent-Revenue-Cap-Methode & $6,15 \%$ & $2,87 \% *$ & $6,90 \% *$ & - \\
\hline Relativer Einzelveräußerungspreis & $60,00 \% *$ & $25,48 \% *$ & $12,93 \% *$ & - \\
\hline Residualwertmethode & $12,31 \%$ & $6,69 \% *$ & $12,07 \% *$ & - \\
\hline
\end{tabular}

Im Hinblick auf die Bilanzierungsalternativen zur Aufteilung des Transaktionspreises auf eigenständige Leistungsverpflichtungen (Tabelle 3.11) äußerten sich mehr als die Hälfte der zum Discussion Paper beteiligten Unternehmen $(60,00 \%)$ zum Bewertungsmaßstab des relativen Einzelveräußerungspreises größtenteils positiv, wobei Unternehmen der Softwarebranche überwiegend die Verbindlichkeit der Regelungen zur Schätzung des Einzelveräußerungspreises, etwa durch Aufnahme einer Anwendungshierarchie, diskutierten ${ }^{364}$.

„Yes we agree that if an entity does not sell a good or service separately it should be able to estimate the standalone selling price of that good or service. We believe there should be a hierarchy for estimation similar to the concepts proposed in EITF 08-1 [...].“(Dell) $)^{365}$

Die Unternehmen der Telekommunikationsbranche äußerten sich hingegen kritisch zur Aufteilung auf Basis geschätzter relativer Einzelveräußerungspreise und forcierten unter Verweis auf die vorherigen Regelungen die Contingent-RevenueCap-Methode und/oder die Residualwertmethode. ${ }^{366}$

„The telecommunications industry currently applies the 'contingent revenue cap' principle, as per paragraph 14 of EITF 00-21 and EITF 08-1, which we believe to be

${ }^{364}$ Die Kookkurrenz zwischen Relativer Einzelveräußerungspreis, Determiniertheit der Regelungsvorschriften und Umfang der Guidance zeigt 14 von 39 Unternehmen, mithin $35,89 \%$. Von den 14 Unternehmen sind 7 Unternehmen aus der Softwarebranche $(50,00 \%)$. 365 Dell (2009), S. 12.

${ }^{366}$ Von 8 Unternehmen sind 7 Unternehmen aus der Telekommunikationsbranche und 1 Unternehmen aus der Softwarebranche. Von den 7 Unternehmen äußerten sich 3 Unternehmen nur zur Residualwertmethode und 4 Unternehmen sowohl zur Residual- als auch zur Contingent-Revenue-Cap-Methode. Das Unternehmen der Softwarebranche äußerte sich zur Residualwertmethode. 
compatible with IAS 18. [...] The Discussion Paper requires arrangement consideration to be allocated between performance obligations on the basis of relative standalone selling prices. We believe that for the telecommunications industry the contingent revenue cap remains appropriate for the following reasons [...]." (Vodafone) $)^{367}$

„Only allowing the allocation of revenue based on the relative standalone selling price of performance obligations is not practical to apply [...]. Specifically we believe that the 'residual' or 'cash cap' method of allocation (as permissible under US GAAP and also compatible under IAS 18) would better reflect the economics of a particular transaction [...].“ $(\mathrm{BT})^{368}$

In den Stellungnahmen der Software- und Telekommunikationsunternehmen werden die branchenspezifischen Besonderheiten der Bilanzierungsalternativen deutlich, wobei die Unternehmen beider Branchen wiederum eine Regelungsausgestaltung zugunsten der bestehenden Bilanzierungspraxis vorantrieben. Andere alternative Bewertungsmaßstäbe wurden zudem von 16,92 \% der Unternehmen hervorgebracht, die fast ausschließlich aus der Fertigungsindustrie stammten. ${ }^{369}$

„Thus, we believe that the allocation basis for the transaction price would be the contractor's best estimates of the costs of various components of a project and their relative costs and margin." (Shaw Group) ${ }^{370}$

Weder der Staff noch die Standardsetzer adressierten im Zuge des Exposure Drafts etwaige alternative Maßstäbe; auch auf eine Umsatzbeschränkung im Sinne der Contingent-Revenue-Cap-Methode wurde nur im Zusammenhang mit der Transaktionspreisbeschränkung eingegangen. ${ }^{371}$ Der Staff und die Boards bestätigten vielmehr unter Bezugnahme auf die Stellungnahmen die Aufteilung auf Basis der relativen (geschätzten) Einzelveräußerungspreise und lehnten eine Festlegung zu verwendender Methoden, mithin auch eine Anwendungshierarchie, sowie die Aufteilung auf Basis der Residualwertmethode ab. ${ }^{372}$ Gleichzeitig eröffneten die Boards in der Basis for Conclusions jedoch die Möglichkeit, die Residualwertmethode als Schätzungsmethode anzuwenden, was als Kompromiss gewertet werden kann bzw. insofern zumindest einzelne Unternehmensbedenken adressierte.

367 Vodafone (2009), S. 2 f.

368 BT (2009), S. 8.

${ }^{369}$ Von 11 Unternehmen sind 9 Unternehmen aus der Fertigungsindustrie $(81,82 \%)$.

${ }^{370}$ Shaw Group (2009), S. 16.

371 Vgl. ED BC (2010), BC95.

${ }^{372}$ Vgl. Technical Staff (2009c), Agenda Paper 3 C/122 C, Rz. 14-29; ED BC (2010), BC112-BC125. 
„Some respondents to the discussion paper suggested that the proposed requirements should permit the residual method [...]. However, the boards noted that the residual method is unnecessary if an entity is required to estimate stand-alone selling prices. [...] Consequently, the boards confirmed their view that the residual method should not be used to allocate the transaction price to separate performance obligations. However, the boards noted that a residual (or reverse residual) technique may be an appropriate method for estimating a stand-alone selling price [...].“(IASB/FASB) ${ }^{373}$

Der deutliche Rückgang der Kommentierungen zum relativen Einzelveräußerungspreis sowie zu alternativen Bewertungsmaßstäben im Exposure Draft spricht für die überwiegende Akzeptanz der Regelungsvorschriften. 28,75 \% der Unternehmen äußerten sich in Bezug auf den relativen Einzelveräußerungspreis zum Regelungsumfang, dabei jedoch unterschiedlich zum Verbindlichkeitsgrad der Regelungen. 374

„Additionally we agree that as long as the method of estimating standalone selling price is consistent and maximizes the use of observable inputs, that no method of estimation should be precluded or prescribed.“ (Northrop Grumman) ${ }^{375}$

„We agree that an entity should allocate the transaction price to all separate performance obligations in a contract in proportion to the standalone selling price (estimated if necessary) [...]. However. we believe additional implementation guidance and examples on suitable estimation methods would be necessary specifically as it relates to suitable estimation methods for biotechnology entities." (Xenoport) ${ }^{376}$

Die Residualwertmethode wurde von 6,69 \% der Unternehmen kommentiert, wobei es sich überwiegend um Unternehmen der Software- und Telekommunikationsbranche handelte, ${ }^{377}$ die die Einführung der Residualwertmethode oder eine Klarstellung zur Verwendung des Residualwerts im Rahmen der Schätzung des Einzelveräußerungspreises forderten.

\footnotetext{
${ }^{373}$ ED BC (2010), BC123-BC125.

${ }^{374}$ Die Kookkurrenz zwischen Relativer Einzelveräußerungspreis, Umfang der Guidance und Determiniertheit der Regelungsvorschriften zeigt 23 von 80 Unternehmen, mithin $28,75 \%$.

375 Northrop Grumman (2010), Attachment, S. 9.

376 Xenoport (2010), S. 2.

${ }^{377}$ Von 21 Unternehmen sind 11 Unternehmen aus der Softwarebranche $(52,38 \%)$ und 7 Unternehmen aus der Telekommunikationsbranche (33,33\%).
} 
„The exposure draft requires the "relative selling price" approach to be followed in allocating the transaction price to the performance obligations. [...] We recommend retaining the accounting policy choice of using either the relative fair value selling price approach or the residual method as currently available under IAS 18 - Revenue.“ (MTN Group) $)^{378}$

„We have noted para BC125 of the ED which states "that a residual (or reverse residual) technique may be an appropriate method [...]". We believe that this guidance should be in the text of the standard rather than in the basis for conclusion." (SAP) ${ }^{379}$

Vor dem Hintergrund der im Standardtext nur marginal erfolgten Änderungen sowie der expliziten Ablehnung der Residualwertmethode durch die Standardsetzer verdeutlichen die Kommentierungen die branchenspezifische Einflussnahme. Die Bezugnahme auf die Klarstellungen in der Basis for Conclusions zeigt dabei die Relevanz der Basis for Conclusions für die Regelungsentwicklung gleichsam die Rekursivität des Standardsetzungsprozesses, insofern als die Unternehmen die Begründungen des IASB einbeziehen. Auch die Stellungnahmen zur Contingent-Revenue-Cap-Methode sind als branchenspezifische Kommentierungen zu werten, da diese fast ausschließlich von Unternehmen der Telekommunikationsbranche stammten, die einheitlich die Übernahme der vorherigen Regelungen zur Beschränkung der Umsatzerfassung empfahlen oder die Vorteile dieser Methode diskutierten.

„We generally agree that the transaction price should be allocated to all separate performance obligations in a contract in proportion to the stand-alone selling price [...]. However, we strongly recommend maintaining the FASB's provision under ASC 60525-30. That is, the amount allocable to a satisfied performance obligation should be limited to the amount that is not contingent upon the fulfilment of additional future performance obligations ("contingent revenue cap")." (Deutsche Telekom) $)^{380}$

„In order to avoid the inconsistencies described above regarding the recognition of handset and service revenues in our industry we suggest that the amount allocable to a satisfied performance obligation should be limited to the amount that is not contingent upon the fulfilment of additional future performance obligations ("contingent revenue cap”).“(Telefónica) ${ }^{381}$

Während im Re-Exposure Draft der Residualwertansatz als Schätzungsmethode in den Standard aufgenommen und diese Änderung in der Basis for Conclusions

\footnotetext{
378 MTN Group (2010), S. 1.

${ }^{379}$ SAP (2010), S. A10.

${ }^{380}$ Deutsche Telekom (2010), S. 12.

381 Telefónica (2010), S. 7.
} 
unter Verweis auf die Stellungnahmen sowie auf bestimmte - besonders für einzelne Branchen relevante - Verträge begründet wurde, lehnten die Standardsetzer die Einführung der Contingent-Revenue-Cap-Methode unter Bezugnahme auf die einschlägigen Kommentierungen ab.

,[T]hose respondents thought that the proposed requirements should clarify how and when an entity could use the residual approach as an estimation method. Therefore, paragraph 73(c) of the proposed requirements specifies the circumstances in which a residual approach would be a suitable method to estimate a stand-alone selling price. In specifying those circumstances, the boards were particularly mindful of the challenges in determining stand-alone selling prices in contracts for intellectual property and other intangible products $[\ldots]$.

However, the boards affirmed their proposal in the 2010 exposure draft not to carry forward the contingent revenue cap for the following reasons [...] Additionally, the boards decided not to introduce an exception to the revenue model for telecommunications and similar contracts because they do not view those contracts to be unique." $(\mathrm{IASB} / \mathrm{FASB})^{382}$

Die vorgenommenen Änderungen im Re-Exposure Draft spiegeln die Reichweite des Unternehmenseinflusses wider; sie stellen insofern einen Kompromiss dar, als die Boards einerseits einheitliche Regelungen zur Aufteilung des Transaktionspreises in Form der relativen Einzelveräußerungspreise ohne industriespezifische Ausnahmeregelung umsetzten, gleichzeitig jedoch anwendungsorientierte Ermessensspielräume in Bezug auf die Schätzungsmethoden ermöglichten.

Die fehlenden Stellungnahmen zu alternativen Bewertungsmaßstäben sowie der Rückgang der Stellungnahmen zum Einzelveräußerungspreis weisen wiederum auf die gestiegene Akzeptanz der Regelungsvorschriften im Re-Exposure Draft hin. Gleichzeitig stieg die Beteiligung zur Residualwertmethode sowie zur Contingent-Revenue-Cap-Methode, wobei überwiegend Unternehmen aus der Telekommunikationsbranche die Bilanzierungsalternativen kommentierten. ${ }^{383}$ Während einzelne Unternehmen dabei weiterhin ausschließlich die Umsetzung der Contingent-Revenue-Cap-Methode forcierten, forderten andere Unternehmen alternativ die Ausweitung des Residualwertansatzes.

\footnotetext{
382 Re-ED BC (2011), BC182, BC196 f.

${ }^{383}$ Im Re-ED sind zum Residualwertansatz 8 von 14 Unternehmen $(57,14 \%)$ und zur Contingent-Revenue-Cap-Methode 7 von 8 Unternehmen $(87,50 \%)$ aus der Telekommunikationsbranche.
} 
„We believe that a restriction on revenue akin to the contingent revenue cap remains the best solution and addresses each of the adverse effects noted above." (Vodafone $)^{384}$

„We request that the final standard retain the current contingent revenue cap. If this is not possible, then we request that additional practical expedients be introduced into the final standard. For instance, we request that the use of the residual technique be specifically allowable for contracts in the telecommunications industry." (Rogers Communication) $)^{385}$

Vor dem Hintergrund des im Re-Exposure Draft unter den Schätzungsmethoden aufgenommenen Residualwertansatzes kann die Forderung zur Umsetzung der Residualwertmethode als Versuch gewertet werden, die Regelungsgestaltung unter der Annahme zu konkretisieren, dass diese Methode gegenüber der Contingent-Revenue-Cap-Methode bei den Standardsetzern als eher durchsetzbar erschien.

Anstelle einer Ausweitung der Residualwertmethode als alternativer Aufteilungsmaßstab bestätigen die Standardsetzer in der Basis for Conclusions zum finalen Standard jedoch die Begrenzung des Ansatzes als Schätzungsmethode und schlossen darüber hinaus einen Residualwert von Null und damit ein der Contingent-Revenue-Cap-Methode ähnliches Ergebnis aus. ${ }^{386}$ Für Anwendungsprobleme aufgrund der fehlenden Contingent-Revenue-Cap-Methode verwiesen die Standardsetzer vielmehr auf die eingeführte Portfolio-Option und adressierten hierbei explizit die Telekommunikationsindustrie, ${ }^{387}$ was als Entgegenkommen gewertet werden kann.

Der Kompromiss zwischen einer konzeptionellen Standardsetzung und einer praxisorientierten Standardanwendbarkeit zeigt sich demzufolge auch bei den Regelungen zur Aufteilung des Transaktionspreises. Wie auch bei den Regelungen zur Vertragszusammenfassung und dem Portfolio-Ansatz sowie den Regelungen zur Aufteilung der Leistungsverpflichtungen werden von den Standardsetzern keine industriespezifischen Ausnahmeregelungen normiert, gleichzeitig erfolgt jedoch eine Einräumung von Ermessensspielräumen bzw. eine anwendungsorientierte Konkretisierung der Regelungsvorschriften. Die Regelungsausgestaltung der untersuchten Bilanzierungsalternativen erfolgte durch umfangreiche Anwendungsleitlinien sowie durch Orientierung an den vorherigen Bilanzierungsvorschriften, was in Einklang mit den von Unternehmen

\footnotetext{
384 Vodafone (2012), S. 2.

385 Rogers Communication (2012), S. 12.

386 Vgl. IFRS 15.BC270-BC273.

${ }^{387}$ Vgl. IFRS 15.BC287-BC293.
} 
adressierten Umsetzungsmöglichkeiten steht. ${ }^{388}$ Die zunehmende Durchsetzung gefundener Bilanzierungslösungen sowie die bereits hinsichtlich des allgemeinen Beteiligungsrückgangs angenommene steigende Akzeptanz der Standardentwürfe im Laufe des Standardsetzungsprozesses wird auch durch den Anstieg der Restkategorie gestützt. ${ }^{389}$

Basierend auf den Ergebnissen zu den Bilanzierungsalternativen und Umsetzungsmöglichkeiten lässt sich hinsichtlich der Rekursivität des Standardsetzungsprozesses feststellen, dass die Stellungnahmen bei der Regelungsüberarbeitung einfließen und vom IASB berücksichtigt werden, die Änderungen und Erwägungen des IASB wiederum in die Stellungnahmen einbezogen und von den Unternehmen konkretisiert werden. Die Teilnahme durch Stellungnahmen am Standardsetzungsprozess ist folglich aus Unternehmensperspektive für die Standardanwendung sinnvoll, da sie eine konkrete Möglichkeit zur Prägung der Regelungsinhalte sowie ihrer Ausgestaltung darstellt und gleichzeitig zu einer frühzeitigen Auseinandersetzung mit möglichen Regelungsänderungen veranlasst. Aus Sicht der Standardsetzer kann einerseits durch die Berücksichtigung der Unternehmensstellungnahmen die Anwendbarkeit der Regelungsvorschriften sichergestellt und der Akzeptanz der gefundenen Regelungen sowie - hierdurch zumindest teilweise - dem Legitimationserfordernis Rechnung getragen werden. Andererseits wirkt sich der Einfluss jedoch auf den konzeptionellen Wandel von Rechnungslegungsvorschriften aus, insofern als eine Standardüberarbeitung in den Grenzen der Standardanwendung verläuft.

\subsubsection{Rolle der Rhetorik bei der Standardsetzung}

\subsection{Häufigkeit verwendeter Überzeugungsarten und rhetorischer Stilmittel}

Neben dem Einfluss auf die inhaltliche Zielrichtung wird im Folgenden die von Unternehmen und Standardsetzern verwendete Rhetorik für die untersuchten Bilanzierungsalternativen ausgewertet, ${ }^{390}$ was Rückschlüsse auf die Reichweite

\footnotetext{
388 Vgl. zur Übersicht der Häufigkeiten der adressierten Umsetzungsmöglichkeiten Appendix, VII.

389 Vgl. zur Übersicht der Entwicklung der Restkategorie Appendix, VII.

${ }^{390}$ Die Subkategorien der Rhetorik wurden nur in Zusammenhang mit den untersuchten Bilanzierungsalternativen codiert. Die Codierung der Rhetorik bei den Standardsetzern erfolgte nur für die von den Standardsetzern verwendeten Überzeugungsarten und rhetorischen Stilmittel, d. h. die Wiedergabe bzw. Zusammenfassung von Unternehmensgründen wurde nicht codiert.
} 
der Überzeugungsstrategien ermöglicht. Im Gegensatz zu den verwendeten Themenübersichten für die inhaltliche Zielrichtung, die die relative Häufigkeit der Unternehmenskommentierungen zeigt, basiert die Auswertung der Rhetorik primär auf der absoluten Codier-Häufigkeit der einzelnen Kategorien in den jeweiligen Kommentierungsphasen. ${ }^{391}$ Hierdurch wird die Verteilung der Überzeugungsarten und rhetorischen Stilmittel unter Berücksichtigung der mehrfachen Verwendung einzelner Überzeugungsmittel eines Unternehmens bzw. der Standardsetzer dargestellt. Die Veranschaulichung der Rhetorik erfolgt wiederum unter Einbezug konkreter Beispiele aus den Stellungnahmen bzw. den Standardsetzungsdokumenten. Zudem wird auch die Kookkurrenz der einzelnen Überzeugungsarten und rhetorischen Stilmittel sowie der inhaltlichen Zielrichtung berücksichtigt, ${ }^{392}$ wodurch Rückschlüsse auf den Zusammenhang zwischen dem Inhalt und der Rhetorik gezogen werden können.

\subsection{Verwendung von Pathos zur Verdeutlichung von Anwendungsproblemen}

Während des Standardsetzungsprozesses von IFRS 15 bedienten sich die Unternehmen (Abbildung 3.4) ${ }^{393}$ bei der Kommentierung sowie die Standardsetzer (Abbildung 3.5) 394 $^{39}$ bei der Rechtfertigung der untersuchten Bilanzierungsalternativen aller drei Überzeugungsarten, wobei Logos und Ethos deutlich häufiger als Pathos eingesetzt wurden.

Die im Vergleich weitaus geringere Verwendung von Pathos kann vor dem Hintergrund der technischen Natur des Standardsetzungsprozesses erklärt werden und deutet darauf hin, dass die im Gegensatz hierzu stehende emotionale Überzeugungskraft sowohl von den Unternehmen als auch den Standardsetzern als eher gering eingestuft wurde.

\footnotetext{
${ }^{391}$ Vgl. zu den Codier-Häufigkeiten und dem Anteil der Unternehmen je Kategorie Appendix, VIII und IX; zu den Codier-Häufigkeiten der Standardsetzer je Kategorie Appendix, X und XI.

${ }^{392}$ Die Kookkurrenz für Kategorien der Rhetorik wurde auf Basis der Codier-Häufigkeiten berechnet, d. h. mehrfache Kookkurrenzen in einer Stellungnahme wurden auch mehrfach gewertet.

393 Vgl. Appendix, VIII.

394 Vgl. Appendix, X.
} 
Häufigkeit der von Unternehmen verwendeten

Überzeugungsarten

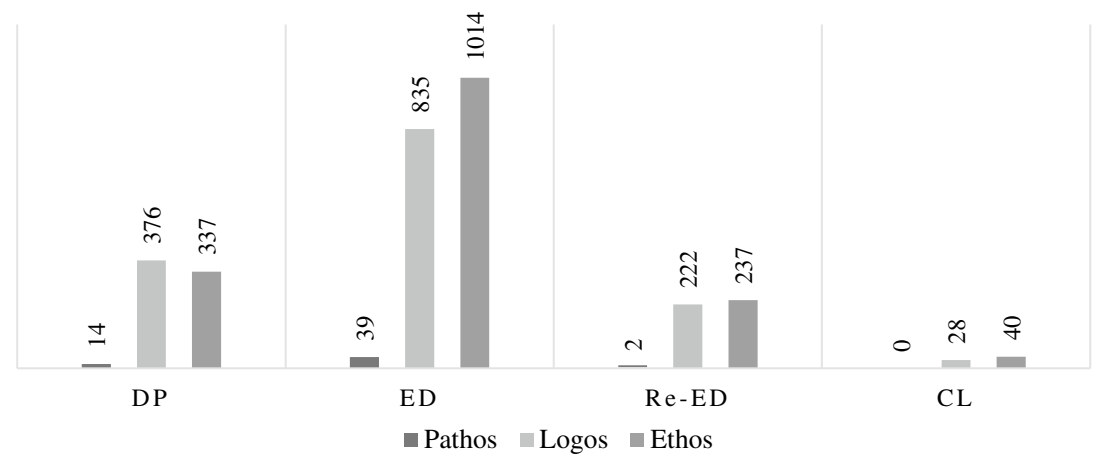

Abbildung 3.4 Codier-Häufigkeit der von Unternehmen verwendeten Überzeugungsarten in der jeweiligen Kommentierungsphase

Häufigkeit der von Standardsetzern verwendeten

Überzeugungsarten

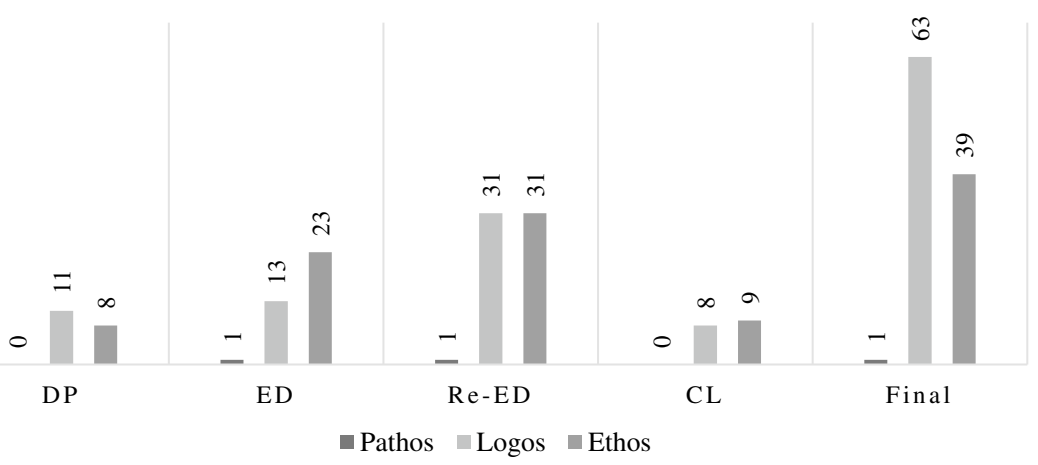

Abbildung 3.5 Codier-Häufigkeit der von den Standardsetzern verwendeten Überzeugungsarten in der jeweiligen Standardsetzungsphase

Im Discussion Paper äußerten sich indes trotzdem 12,31 \% der Unternehmen in Form von Übertreibungen ${ }^{395}$ zur Unmöglichkeit der Anwendbarkeit der

395 Vgl. Appendix, IX. 
Regelungen zur Aufteilung der Leistungsverpflichtungen und des Transaktionspreises, ${ }^{396}$ wodurch anstelle von rational begründbaren Praktikabilitätsbedenken vielmehr die Nicht-Umsetzbarkeit der Regelungsvorschläge auf emotionaler Ebene betont wurde.

„The number of performance obligations that would be identified, combined with the sheer volume of low value transactions, results in a number of relative standalone sales price that is simply impossible for a telecommunications company to apply.“ (Deutsche Telekom) ${ }^{397}$

Auch im Exposure Draft wurde Pathos überwiegend im Rahmen der Kommentierung eines vertragsübergreifenden Ansatzes bzw. der Ablehnung der Aufteilungskriterien eingesetzt. ${ }^{398}$ Hierbei handelte es sich fast ausschließlich um US-amerikanische Unternehmen der Fertigungsindustrie, ${ }^{399}$ die neben der unmöglichen Anwendbarkeit der Regelungen die ökonomischen Konsequenzen einer Standardänderung in Bezug auf die ohnehin schwierige wirtschaftliche Lage hervorhoben und hierdurch die Regelungskonsequenzen an einen Gefühlszustand anknüpften. ${ }^{400}$

„Like the rest of the economy the construction industry is on hard times; however the construction economy is suffering far worse than our United States economy. This would not be a good time to introduce additional economic hardship to the construction industry.“ (Emerick) ${ }^{401}$

„The cost of new software and additional accounting staff expenses will be an onerous burden on, particularly, smaller private companies. This will close some companies' doors and be a huge burden on our already struggling industry.“ (Vance Brown) ${ }^{402}$

Die Verwendung von Pathos erfolgte im Re-Exposure Draft durch lediglich zwei Unternehmen aus der Telekommunikationsbranche, die ihre Bedenken zur

\footnotetext{
${ }^{396}$ Die Kookkurrenz zwischen Pathos und Hyperbel zeigt 9 von 14 Codierungen, mithin $64,29 \%$.

${ }^{397}$ Deutsche Telekom (2009), S. 10.

398 Die Kookkurrenz zwischen Pathos und den Subkategorien zur Aufteilung der Leistungsverpflichtungen zeigt 26 von 39 Codierungen, mithin $66,67 \%$.

${ }^{399}$ Von 30 Unternehmen sind 27 Unternehmen aus der Fertigungsindustrie und USamerikanischer Herkunft $(90,00 \%)$.

400 Vgl. Stenka (2014), S. 22.

401 Emerick (2010), S. 1.

402 Vance Brown (2010), S. 3.
} 
Anwendbarkeit des Standards bzw. ihr Anliegen auf mögliche Änderungen zugunsten der Aufnahme einer Contingent-Revenue-Cap-Methode mit Nachdruck äußerten.

„As a result of the field-testing we feel justified in our concerns that the proposed model will be impossible to apply for telecom operators, given the tremendous volume of transactions we face every day." (Deutsche Telekom) ${ }^{403}$

Pathos wurde demnach ausschließlich bei Bilanzierungsalternativen eingesetzt, die Alternativvorschläge gegenüber den von den Standardsetzern vorgeschlagenen Regelungsvorschriften darstellten und die Anwendbarkeit der Regelungen adressierten. Dies diente dazu, die Überlegenheit des Regelungsvorschlags gegenüber der bestehenden Bilanzierungspraxis in Frage zu stellen und die Aufmerksamkeit der Standardsetzer auf die praktische Umsetzbarkeit der Regelungen zu lenken.

Die Überzeugungskraft von Pathos, insbesondere von dem Verweis auf die unmögliche Anwendbarkeit, wird auch in den Basis for Conclusions zum Exposure Draft, Re-Exposure Draft und finalen Standard deutlich, in denen die Standardsetzer die Ausgestaltung der Anforderung der eigenständigen Funktion auf Basis der in alten Standards bestehenden Regelungen des ,value to the customer on a standalone basis" ablehnten.

„It would be difficult, if not impossible, for an entity to know the customer's intentions in any given contract.“ (IASB/FASB) ${ }^{404}$

Die Verwendung der Übertreibung kann als Überzeugungsversuch des Standardsetzers gewertet werden, in diesem Fall jedoch um die Überlegenheit des Regelungsvorschlags gegenüber den vorherigen Standards zu betonen. Gleichzeitig wird die Anschlussfähigkeit von Pathos deutlich, das zur Überzeugung der Nicht-Umsetzung eines Regelungsvorschlags eingesetzt wurde.

\subsection{Verwendung von Logos zur Demonstration der rational underpinnings}

Im Gegensatz zu der geringen Nutzung von Pathos zeichnen sich die Unternehmensstellungnahmen durch eine umfangreiche Verwendung von Logos aus, wobei eine Stellungnahme in der Regel mehrfache Begründungen beinhaltete. Während

403 Deutsche Telekom (2012), S. 2.

${ }^{404}$ ED BC (2010), BC52; Re-ED BC (2011), BC76; IFRS 15.BC101. 
Logos im Discussion Paper die am häufigsten genutzte Überzeugungsart darstellte, wurde Ethos in allen anderen Standardentwurfsphasen häufiger verwendet (Abbildung 3.4). Die Erzeugung von Rationalität erfolgte während des Standardsetzungsprozesses überwiegend durch konzeptionelle und ökonomische Gründe, die in allen Phasen etwa gleich häufig, im Exposure Draft und Re-Exposure Draft jedoch mit leichter Tendenz zu ökonomischen Gründen, eingesetzt wurden; Beispiele wurden indes fast weniger als halb so oft verwendet (Abbildung 3.6) ${ }^{405}$. Die in etwa gleiche Verteilung von konzeptionellen und ökonomischen Gründen bestätigt die Ergebnisse der Lobbying-Literatur, wonach Unternehmen ihre Argumentation sowohl auf ökonomische Konsequenzen als auch auf konzeptionelle Rechtfertigungen stützen. ${ }^{406}$

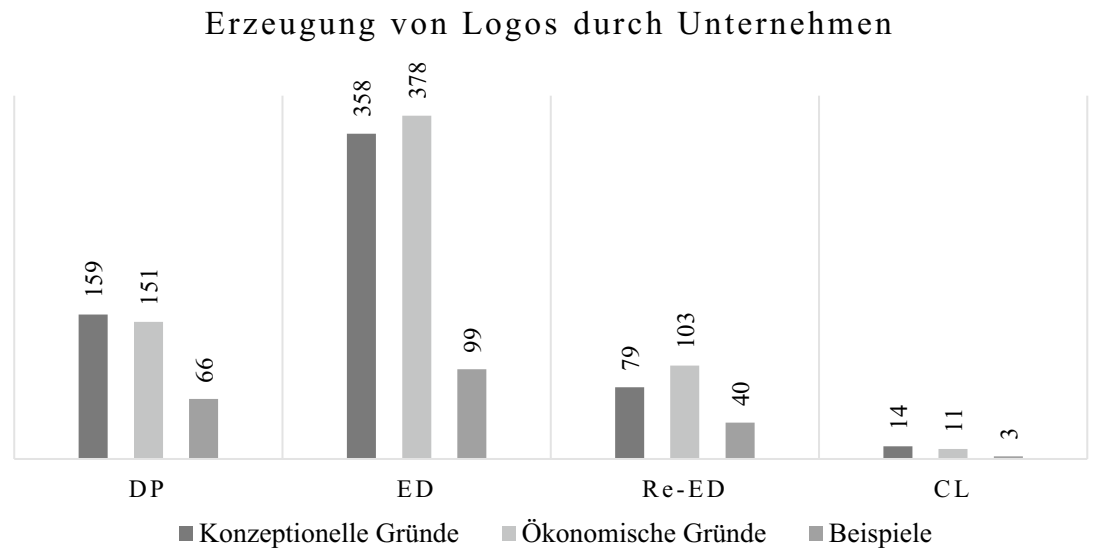

Abbildung 3.6 Codier-Häufigkeit der von Unternehmen verwendeten Subkategorien der Überzeugungsart Logos in der jeweiligen Kommentierungsphase

Von den Standardsetzern wurde Logos im Vergleich zu den anderen Überzeugungsarten im Discussion Paper und insbesondere im finalen Standard am häufigsten verwendet (Abbildung 3.5). Während für die Erzeugung von Rationalität im Discussion Paper und Exposure Draft sowohl konzeptionelle und ökonomische Gründe als auch Beispiele etwa gleich häufig eingesetzt wurden, dominierten ab dem Re-Exposure Draft die konzeptionellen Begründungen

${ }^{405}$ Vgl. Appendix, VIII.

${ }^{406}$ Vgl. Jupe (2000), S. 349-354; Stenka/Taylor (2010), S. 119-124. 
(Abbildung 3.7) ${ }^{407}$. Dies steht in Einklang mit der vom Standardsetzer verfolgten, auf dem Rahmenkonzept basierenden, deduktiven Standardsetzung, zeigt gleichzeitig jedoch auch die, die ökonomischen Folgen berücksichtigende, induktive Komponente der Standardsetzung.

Erzeugung von Logos durch Standardsetzer

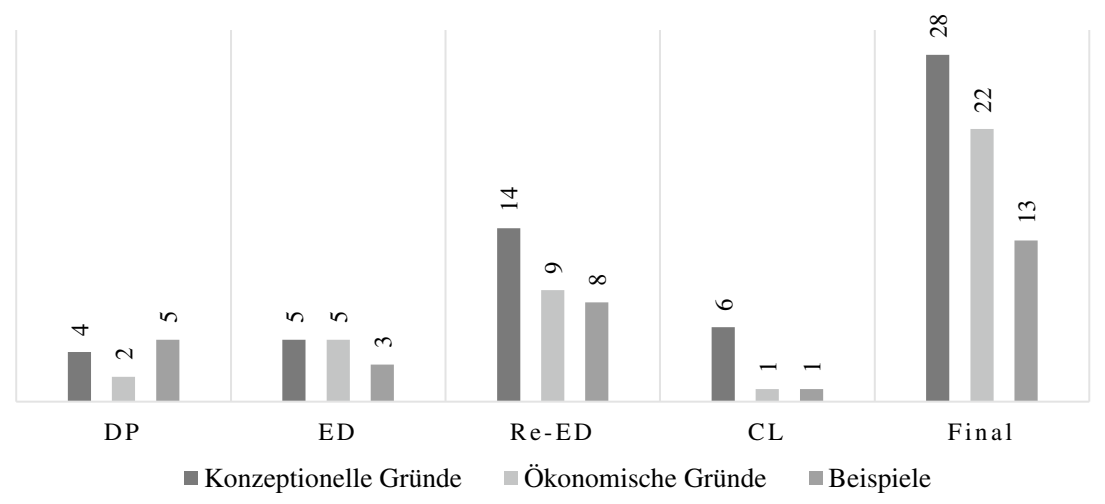

Abbildung 3.7 Codier-Häufigkeit der von den Standardsetzern verwendeten Subkategorien der Überzeugungsart Logos in der jeweiligen Standardsetzungsphase

Als konzeptionelle Gründe wurden von Unternehmen in allen Kommentierungsphasen die Entscheidungsnützlichkeit sowie die Vergleichbarkeit am häufigsten angeführt; die Begründungen zu den Clarifications beschränkten sich auf die Vergleichbarkeit sowie die Konsistenz der Regelungsausgestaltung. ${ }^{408}$ Obwohl die Vergleichbarkeit seit der Rahmenkonzeptüberarbeitung (2010) nur eine ergänzende Anforderung darstellt und bereits im alten Rahmenkonzept der Konkretisierung der Entscheidungsnützlichkeit diente, dominierte sie in allen Standardentwurfsphasen zu IFRS 15 als konzeptionelle Begründung, ${ }^{409}$ was auf die von Unternehmen wahrgenommene höhere Überzeugungskraft dieses Kriteriums hindeutet. Die Begründung der jeweiligen Bilanzierungsalternative anhand

\footnotetext{
407 Vgl. Appendix, X.

408 Vgl. Appendix, VIII.

${ }^{409}$ Vgl. auch Wüstemann (2018), S. 25.
} 
der Vergleichbarkeit erfolgte dabei weniger im Kontext der Entscheidungsnützlichkeit als vielmehr in Zusammenhang mit ökonomischen Gründen sowie unter Verwendung von Beispielen. ${ }^{410}$

„As a result, it is not clear that the guidance will be able to be implemented in an objective, practical and consistent manner across all entities." (CSC) $)^{411}$

„We believe that splitting E\&C contracts into different performance obligations would be arbitrary. [...] One contractor might classify, for example, scaffolding and mobilization as deliverables; whereas another might classify them as indirect costs and spread them among other identified deliverables, leading to inconsistent revenue recognition between the two contracts." (Kiewit) ${ }^{412}$

Die Vergleichbarkeit der Informationsvermittlung wird insofern an die vergleichbare Anwendung der Regelungsvorschrift geknüpft. Dies kann wiederum als Überzeugungsstrategie gewertet werden, den Fokus auf die Anwendbarkeit der Regelungen konzeptionell zu begründen.

Die Entscheidungsnützlichkeit wurde indes sowohl zusammen mit ökonomischen Gründen als auch in Verbindung mit anderen konzeptionellen Gründen angeführt, ${ }^{413}$ was die Auslegungsoffenheit der Zwecksetzung verdeutlicht.

„The proposed standard currently calls for an entity to identify all of the elements in a contract [...] and then aggregate them into performance obligations [...]. The practical implementation issues are highly significant and may not lead to useful information for decision making." (CSC) $)^{414}$

,[T]he exposure draft appears to ignore the intent of the parties who negotiated the contract, focusing instead on the unrelated possibility that the good or service "can" be sold separately. We believe this approach will produce financial results that are not

${ }^{410}$ Die Kookkurrenz von Vergleichbarkeit und Entscheidungsnützlichkeit zeigt im DP 4 von 57 (7,02 \%), im ED 13 von 157 (8,28 \%) und im Re-ED 7 von 36 Codierungen (19,44\%). Die Kookkurrenz von Vergleichbarkeit und den Subkategorien der ökonomischen Gründe zeigt im DP 14 von 57 (24,56 \%), im ED 22 von 157 (14,01\%) und im Re-ED 7 von 36 Codierungen $(19,44 \%)$. Die Kookkurrenz von Vergleichbarkeit und Beispiele zeigt im DP 8 von $57(14,04 \%)$, im ED 15 von 157 (9,55\%) und im Re-ED 4 von $36(11,11 \%)$ Codierungen. ${ }^{411}$ CSC (2010), S. 5.

412 Kiewit (2009), S. 4.

${ }^{413}$ Die Kookkurrenz von Entscheidungsnützlichkeit und den Subkategorien der ökonomischen Gründe als auch die Kookkurrenz zwischen Entscheidungsnützlichkeit und den Subkategorien der konzeptionellen Gründe zeigt jeweils 47 von 173 Codierungen, mithin jeweils $27,17 \%$.

${ }^{414}$ CSC (2010), S. 1 f. 
relevant and could be misleading to financial statement users of companies involved in long-term construction projects." (National Oilwell Varco) ${ }^{415}$

Auch die Standardsetzer begründeten die Regelungsvorschläge häufiger unter Bezugnahme auf die Vergleichbarkeit als mit der Entscheidungsnützlichkeit; ${ }^{416}$ vor dem Hintergrund der verfolgten deduktiven Standardsetzung wäre indes eine stärker zweckgerichtete Normbegründung erwartbar gewesen. Die Notwendigkeit neuer Regelungsvorschläge wird dabei durch die "diversity in practice“ gerechtfertigt. ${ }^{417}$ So begründeten die Standardsetzer verschiedene Standardsetzungsentscheidungen mit der im Vergleich zu den vorherigen Regelungen gesteigerten Vergleichbarkeit bzw. in den Clarifications und im finalen Standard mit der im Gegensatz zur Aufnahme einer Ausnahmeregelung höheren Vergleichbarkeit.

„The boards observed that even though IFRS 15 may result in significant differences in the allocation of the transaction price to performance obligations [...] in some industries, the change was necessary to provide greater consistency in the recognition of revenue across industries." (IASB/FASB) $)^{418}$

Dieses Ergebnis legt nahe, dass die Überzeugungskraft des Vergleichbarkeitsarguments auch von den Standardsetzern angenommenen wurde, was insoweit in Einklang mit den Unternehmensbegründungen steht und folglich auf die höhere Anschlussfähigkeit dieser Begründung im Vergleich zur Entscheidungsnützlichkeit hindeutet.

Als konzeptionelle Begründung wurde von Unternehmen zudem die konsistente Ausgestaltung der Regelungsvorschläge angeführt, die im Gegensatz zum Argument der Vergleichbarkeit jedoch kaum durch ökonomische Gründe konkretisiert wurde. ${ }^{419}$ Während im Discussion Paper fast ausschließlich Unternehmen der Telekommunikationsbranche die Ablehnung zur Aufteilung des Transaktionspreises auf Basis relativer Einzelveräußerungspreise unter Verweis auf bestehende

\footnotetext{
415 National Oilwell Varco (2010), S. 2.

416 Vgl. Appendix, X.

417 Vgl. Young (1994), S. 86 f. (auch Zitat, S. 86).

418 IFRS 15.BC476.

${ }^{419}$ Die Kookkurrenz von Konsistenz und den Subkategorien der ökonomischen Gründe zeigt 6 von 47 Codierungen, mithin $12,77 \%$.
} 
Inkonsistenzen begründeten, wurde im Exposure Draft die Konsistenz mehrheitlich bei der Ausgestaltung der Regelungen zur Vertragsaufteilung bzw. -zusammenfassung im Verhältnis zum Distinct-Kriterium angeführt. ${ }^{420}$

„We see some inconsistencies in the underlying principles in the DP, as the contract is used as the base to identify performance obligations but the prices agreed in that contract are not used for the allocation of the transaction price to such obligations." (Telefónica) $^{421}$

„As written, we are concerned it could result in a proliferation of segmenting that would be inconsistent with the spirit of the related guidance on identifying performance obligations." (Bombardier) ${ }^{422}$

Auch im Re-Exposure Draft betonten Unternehmen der Telekommunikationsbranche die zum Rahmenkonzept inkonsistente Vermögenswerterfassung ohne eine Contingent-Revenue-Cap-Methode.

„Our main arguments for introducing the contingent revenue cap into the proposed model were the following: The contract asset recognised for the amount exceeding the non-contingent amount does not fulfil the definition of an asset according to the Conceptual Framework." (Deutsche Telekom) ${ }^{423}$

Das Argument der Konsistenz wurde von den Standardsetzern im Laufe des Standardsetzungsprozesses mit zunehmender Häufigkeit verwendet, was auch darauf zurückgeführt werden kann, dass es zur Adressierung der Kommentierungen der Telekommunikationsbranche zur Umsetzung der Contingent-RevenueCap-Methode diente. ${ }^{424}$ Die Nicht-Umsetzung wurde sowohl mit der inneren Konsistenz des Standards als auch mit der konsistenten Ausgestaltung zum Rahmenkonzept begründet.

${ }^{420}$ Im DP sind 4 von 5 Unternehmen aus der Telekommunikationsbranche (80,00\%); die Kookkurrenz von Konsistenz und den Subkategorien zur Aufteilung des Transaktionspreises zeigt 4 von 5 Codierungen, mithin 80,00 \%. Die Kookkurrenz im ED zwischen Konsistenz und Aufteilung sowie Zusammenfassung von Verträgen zeigt 19 von 36 Codierungen, mithin $52,78 \%$.

421 Telefónica (2009), S. 13.

422 Bombardier (2010), S. 2.

423 Deutsche Telekom (2012), S. 11.

${ }^{424}$ Die Kookkurrenz zwischen Konsistenz und Contingent-Revenue-Cap-Methode zeigt im Re-ED 3 von 5 Codierungen, mithin 60,00\%, und im finalen Standard 3 von 7 Codierungen, mithin $42,86 \%$. 
,[L]imiting the amount of consideration that can be allocated to a satisfied separate performance obligation is tantamount to cash-basis accounting and does not meet the core principle of the proposed requirements.

[...] recognising a contract asset in the situation described in paragraph BC195 is appropriate because the entity clearly has a valuable contractual right as a result of satisfying a performance obligation and that right meets the definition of an asset.“ $(\text { IASB/FASB })^{425}$

Trotz gleicher konzeptioneller Gründe wurde von den Standardsetzern ein gegensätzliches Ergebnis begründet. Dieses steht jedoch in Einklang mit der Zielsetzung der Entwicklung eines konsistenten, branchenübergreifenden Umsatzerfassungsmodells. ${ }^{426}$ Die Überzeugungskraft des Konsistenzarguments beruht insofern auf einer deduktiv-logischen Schlussfolgerung.

Eine weitere konzeptionelle Begründung stellte die Anforderung der Verlässlichkeit dar, die von Unternehmen im Discussion Paper deutlich häufiger als die Anforderung der glaubwürdigen Darstellung verwendet wurde; im Exposure Draft und Re-Exposure Draft zeichnete sich hingegen eine im Vergleich zunehmende Begründung auf Basis der glaubwürdigen Darstellung $a b,{ }^{427}$ was mit den Änderungen der Rahmenkonzeptanforderungen in Einklang steht. Im Widerspruch zur Anschlussfähigkeit dieser Rahmenkonzeptänderungen steht indes die im Laufe des Standardsetzungsprozesses kontinuierliche Verwendung der Verlässlichkeit als Begründung von einzelnen Unternehmen, insbesondere aus der Telekommunikationsbranche.

„Furthermore, the proposed model involves the use of estimations to a greater extent thereby increasing complexity and potentially decreasing reliability and comparability of the information." (Telefónica) ${ }^{428}$

Auch die Standardsetzer benutzten das Argument der Verlässlichkeit, jedoch ausschließlich zur Begründung des Residualwerts als Schätzmethode.

„In those circumstances, the most reliable way of determining the stand-alone selling price in the contract will often be to use a residual approach." (IASB/FASB) ${ }^{429}$

\footnotetext{
425 Re-ED BC (2011), BC196.

426 Vgl. Wüstemann (2018) S. 32.

${ }^{427}$ Vgl. Appendix, VIII; auch Wüstemann (2018), S. 25.

428 Telefónica (2012), S. 2.

${ }^{429}$ Re-ED BC (2011), BC182.
} 
Im Vergleich zur Verlässlichkeit wurde die glaubwürdige Darstellung als Argument durch die Standardsetzer während des gesamten Standardsetzungsverfahrens wesentlich häufiger verwendet. ${ }^{430}$ Die Begründung anhand der Verlässlichkeit deutet daher auf die Überzeugungskraft auf Basis eines punktuell geteilten Verständnisses der Anforderung in Bezug auf Bewertungsunsicherheiten hin. Gleichzeitig zeigt die häufigere Begründung anhand der glaubwürdigen Darstellung, dass die Standardsetzer einerseits die Rahmenkonzeptänderungen bereits im Discussion Paper antizipierten oder die Bedeutung von Verlässlichkeit bereits als glaubwürdige Darstellung interpretierten, andererseits der Anforderung eine für die Standardsetzung wesentliche Bedeutung beimessen. Die Überzeugungskraft beruht insofern auf der Unbestimmtheit bzw. der Interpretationsoffenheit der Anforderung, die eine subjektiv geprägte Begründung ermöglicht bzw. eine subjektiv geprägte Begründungserschließung erfordert. ${ }^{431}$

Im Hinblick auf die ökonomischen Gründe verwendeten Unternehmen in allen Standardentwurfsphasen am häufigsten das Argument der Praktikabilität; in Bezug auf die Kosten-Nutzen-Erwägungen wurde das Kostenargument häufiger angeführt. ${ }^{432}$ Durch die Begründungen wurden die ökonomischen Folgen der Regelungsvorschläge betont, die vereinzelt durch die Verwendung von Pathos verstärkt wurden.

„A contract can indeed bundle separate performance obligations, and unbundling all separate performance obligations would be unnecessarily complex." (Roche) $)^{433}$

,[I]n theory, we do not have an issue with allocating the transaction price to all separate performance obligations. Our concem again is with the practical application of this principle." (Alcatel Lucent) ${ }^{434}$

„We are concerned that the "performance obligation" approach to revenue recognition will not improve the existing financial reports, for our particular industry, and will increase the cost of financial reporting and reduce the availability of surety credit to an industry that is already suffering amidst an economic downturn." (Hatcher Construction $)^{435}$

\footnotetext{
${ }^{430}$ Vgl. Appendix, X.

${ }^{431}$ Vgl. auch La Torre u. a. (2020), S. 14 f.; Wüstemann (2018), S. 32.

432 Vgl. Appendix, VIII.

${ }^{433}$ Roche (2009), S. 3.

434 Alcatel Lucent (2010), S. 3.

435 Hatcher Construction (2010), S. 1.
} 
Als Kostengründe wurden ganz allgemein neben höheren administrativen Kosten der Umstellung im Speziellen die Kosten für zusätzliche Berichterstattungen, Kosten aufgrund von Änderungen und Umstellungen von IT-Systemen, die Zunahme etwaiger Steuereffekte sowie höhere Personal-, Kreditaufnahme- sowie Prüfungskosten genannt. ${ }^{436}$

Auch die Standardsetzer verwendeten ökonomische Gründe zur Rechtfertigung der Regelungsvorschläge, wobei ebenso wie bei Unternehmen die Praktikabilität der am häufigsten genannte Grund darstellte und das Kostenargument wiederum häufiger als das Nutzenargument angeführt wurde. ${ }^{437}$

„Consequently, when considering how entities across various industries should identify separate performance obligations, the boards' objective was to develop requirements that would result in an entity recognising revenue and profit margins in a manner that faithfully depicts the transfer of goods or services to the customer and that would be practical.“ (IASB/FASB) ${ }^{438}$

„These requirements were developed on the basis of extensive consultation and attempts to separate contracts in a meaningful and cost-effective way with intuitive outcomes." (IASB/FASB) $)^{439}$

Durch die Begründung anhand ökonomischer Gründe wird dem Erfordernis der Berücksichtigung der Regelungsanwendbarkeit Rechnung getragen, was gleichzeitig die Beachtung von Unternehmensinteressen impliziert. Die Argumentation auf Basis ökonomischer Gründe bezieht sich folglich auf die praktische Umsetzbarkeit sowie die Kosteneffizienz neuer Regelungen und erlangt ihre Überzeugungskraft insofern durch eine induktiv-logische Schlussfolgerung.

Neben konzeptionellen und ökonomischen Gründen nutzten sowohl Unternehmen als auch Standardsetzer Beispiele zur Erläuterung der einzelnen Bilanzierungsalternativen. Während die Unternehmen Beispiele am Anfang des Standardsetzungsprozesses, d. h. im Discussion Paper und Exposure Draft, häufiger verwendeten, zeichnete sich bei den Standardsetzern eine Zunahme beispielhafter Begründungen im Laufe des Prozesses ab. ${ }^{440}$ Die Beispiele fungierten dabei als anschauliche Nachweise und trugen auf diese Weise zur Schlüssigkeit der Argumentation bei.

\footnotetext{
436 Vgl. auch Wüstemann (2018), S. 27 f.

437 Vgl. Appendix, X.

${ }^{438}$ ED BC (2010), BC48.

${ }^{439}$ IFRS 15.BC471.

${ }^{440}$ Vgl. Appendix, VIII und X.
} 
„Specifically, the functional interdependence between deliverables within distinct arrangements is a factor that is not sufficiently covered by the current proposal. For example, consider two arrangements entered into and fulfilled in parallel with one deliverable each where the prices are fixed and equal the respective list prices but where the deliverable of the one arrangement is essential to the deliverable of the other arrangement [...].“(SAP) ${ }^{441}$

„The boards were concerned that requiring an entity to account separately (and estimate a stand-alone selling price) for a good or service that is not capable of providing a benefit to the customer might result in information that would not be relevant to users of financial statements. For example, if an entity transferred a machine to the customer, but the machine is only capable of providing a benefit to the customer after an installation process that only the entity can provide, the machine would not be distinct." (IASB/FASB) $)^{442}$

Die Erzeugung von Logos basiert folglich auf der Verwendung konzeptioneller und ökonomischer Gründe sowie von Beispielen, mithilfe derer die ,rational underpinnings of the technical aspects“443 der Normermittlung demonstriert werden.

\subsection{Verwendung von Ethos zur Legitimation formaler und inhaltlicher Standardsetzungskompetenz}

Neben Logos setzten Unternehmen auch Ethos häufig als Überzeugungsmittel ein, das ab dem Exposure Draft die am häufigsten verwendete Überzeugungsart darstellte (Abbildung 3.4). Glaubwürdigkeit wurde in allen Standardentwurfsphasen am häufigsten in Form von Expertise erzeugt; Respekt, Gemeinsamkeiten und Autorität wurden in etwa halb so häufig vermittelt (Abbildung 3.8) ${ }^{444}$. Eine Bezugnahme auf Erfolge oder Fehler des Unternehmens, die sie glaubwürdiger in Bezug auf die Standardsetzung erscheinen lassen, erfolgte hingegen nicht, was sich vor dem Hintergrund einer unerwünschten Vermittlung von Eigennutz bzw. Inkompetenz erklären lässt.

Von den Standardsetzern wurde Ethos im Vergleich zu den anderen Überzeugungsarten im Exposure Draft und den Clarifications am häufigsten verwendet; abgesehen vom Exposure Draft und dem finalen Standard war die Differenz zwischen Ethos und Logos jedoch in allen anderen Standardentwurfsphasen gering (Abbildung 3.5). Die Erzeugung von Glaubwürdigkeit erfolgte im gesamten

\footnotetext{
441 SAP (2010), S. A1.

442 IFRS 15.BC97.

${ }^{443}$ La Torre u. a. (2020), S. 13.

444 Vgl. Appendix, VIII.
} 


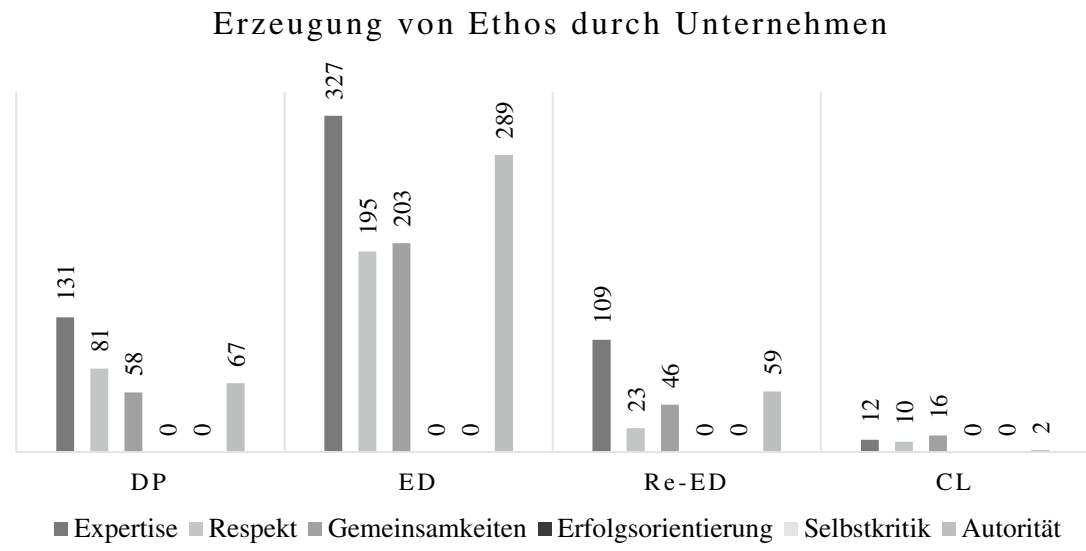

Abbildung 3.8 Codier-Häufigkeit der von Unternehmen verwendeten Subkategorien der Überzeugungsart Ethos in der jeweiligen Kommentierungsphase

Standardsetzungsprozess am häufigsten durch die Vermittlung von Respekt und Autorität, die in den Standardentwurfsphasen in etwa gleich häufig, im finalen Standard mit überwiegender Häufigkeit von Respekt verwendet wurden (Abbildung 3.9 $)^{445}$. Am Anfang eines Standardentwurfs bzw. im finalen Standard legten die Standardsetzer zudem die erfolgsversprechenden Standardsetzungsziele sowie die Defizite der alten Regelungen dar, was als Strategie zur Überzeugung von der Notwendigkeit der Standardüberarbeitung gewertet werden kann.

„The next section considers some of the problems in US GAAP and IFRSs.“ $(\text { IASB/FASB })^{446}$

„Because revenue is a crucial number to users of financial statements, the boards think that having a common standard on revenue for IFRSs and US GAAP is an important step towards achieving the goal of a single set of high quality global accounting standards." (IASB/FASB $)^{447}$

Das Aufzeigen von Gemeinsamkeiten sowie der Expertise der Board-Mitglieder erfolgte hingegen nicht, was indes aufgrund der formal bestehenden Standardsetzungsbefugnis gerechtfertigt werden kann.

\footnotetext{
445 Vgl. Appendix, X.

${ }^{446} \mathrm{DP}(2008), 1.2$.

${ }^{447}$ ED BC (2010), BC08.
} 


\section{Erzeugung von Ethos durch Standardsetzer}

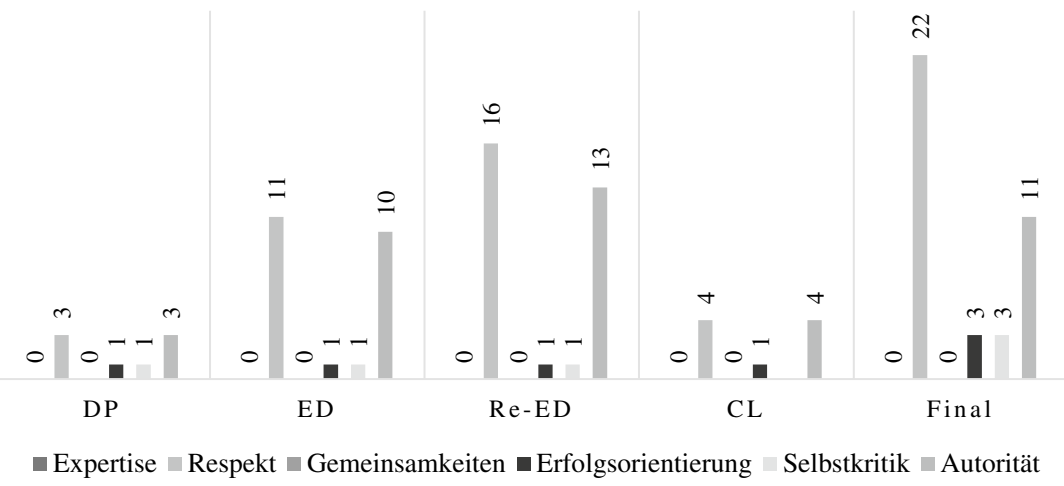

Abbildung 3.9 Codier-Häufigkeit der von den Standardsetzern verwendeten Subkategorien der Überzeugungsart Ethos in der jeweiligen Standardsetzungsphase

Im Gegensatz zu den Standardsetzern lässt sich für Unternehmen der Vermittlung von Expertise sowie Gemeinsamkeiten aufgrund der Häufigkeit der Verweise die Annahme einer hohen Überzeugungskraft zuschreiben. Die Darstellung des Fachwissens äußerte sich durch unternehmens- und/oder industriespezifische Erläuterungen der einzelnen Bilanzierungsalternativen und erfolgte sowohl in Zusammenhang mit Praktikabilitätsgründen als auch insbesondere unter Verwendung von Beispielen. ${ }^{448}$

„We believe that a contract by contract assessment of the treatment of these materials [...] on this basis would be burdensome for any member of the E\&C industry that operates in multiple jurisdictions (in fact, individual contracts can be constructed in multiple jurisdictions), and would result in otherwise similar transactions receiving different accounting." (Kiewit) $)^{449}$

„As written, it is also unclear whether paragraph 29 would apply to the software/services industry or whether it only applies to the construction of physical assets

\footnotetext{
${ }^{448}$ Die Kookkurrenz zwischen Expertise und Praktikabilität zeigt im DP 32 von 131 $(24,43 \%)$, im ED 36 von 327 (11,01\%), im Re-ED 17 von 109 (15,60\%) und in den CL 0 von $12(0 \%)$ der Codierungen. Die Kookkurrenz zwischen Expertise und Beispielen zeigt im DP 38 von 131 (29,01 \%), im ED 72 von 327 (22,02 \%), im Re-ED 32 von 109 (29,36\%) und in den CL 2 von $12(16,67 \%)$ der Codierungen.
}

${ }^{449}$ Kiewit (2009), S. 3. 
such as buildings. For example, IBM sells "bundles" of software and services in which the software is significantly modified to meet the needs of the customer." (IBM) ${ }^{450}$

Hierdurch wurde Glaubwürdigkeit einerseits auf Basis von unternehmensspezifischem Fachwissen in Form von praktischen Erfahrungen sowie andererseits auf Basis der industriespezifischen Stellung der Unternehmen durch die Vermittlung branchenübergreifender Ansichten erzeugt. Der Fokus wurde insoweit weniger auf das Eigeninteresse, sondern vielmehr auf das jeweilige „,industry or business setting" als Wissensbasis der Kommentierung gelegt. ${ }^{451}$

Unternehmen verwiesen zudem meist am Anfang ihrer Stellungnahmen - teils unter Betonung der Unternehmensstellung - auf Gemeinsamkeiten in Bezug auf die geteilten Ziele der Standardsetzer.

„We support the Boards' efforts to simplify the complex framework of revenue recognition standards currently in place under U.S. Generally Accepted Accounting Principles (U.S. GAAP), and we agree that the goal of a single standard for revenue recognition across all industries is a worthy objective." (General Dynamics) ${ }^{452}$

„NTT DOCOMO, INC. is a large mobile telecommunication services provider with operations principally in Japan. Our shares have been listed on the First Section of the Tokyo Stock Exchange. Our shares have been also quoted and traded through the New York Stock Exchange and the London Stock Exchange. Therefore, we are preparing our consolidated financial statements in accordance with accounting principles generally accepted in the United States of America ("U.S. GAAP"). In this regard, we fully support the International Accounting Standards Board and the Financial Accounting Standards Board's intention to develop a single principle for revenue recognition.“ $(\text { NTT Docomo) })^{453}$

Der Verweis auf die Unternehmensstellung kann dabei als Versuch gewertet werden, das eigene Renommee zu betonen und hierdurch die Glaubwürdigkeit der Stellungnahme zu erhöhen, ist jedoch letztlich von der Auffassung der Standardsetzer abhängig. Das schmeichelhafte Vorgehen erzeugt insbesondere im Hinblick auf die Vermittlung der geteilten Zielsetzung indes ein Bild des guten Willens

\footnotetext{
${ }^{450}$ IBM (2012), S. 9.

${ }^{451}$ Vgl. auch Stenka (2014), S. 21 (auch Zitat).

452 General Dynamics (2009), S. 1.

${ }^{453}$ NTT Docomo (2010), S. 1.
} 
und der Aufgeschlossenheit, ${ }^{454}$ das vor allem im Fall kritischer Kommentierungen zur Überzeugung von der Legitimität ${ }^{455}$ der Unternehmensäußerungen für Standardsetzungsfragen diente.

Die Erzeugung von Ethos erfolgte zudem durch die Vermittlung von Respekt gegenüber den Standardsetzern. Durch eine reflexive Würdigung der Regelungsvorschläge bzw. der Standardsetzungsentscheidungen äußerten Unternehmen ihre Ansichten dabei in Relation zu den Standardsetzern.

„The Boards have defined a performance obligation as "a promise in a contract with a customer to transfer an asset (such as a good or a service) to that customer." We generally agree with the Boards' definition of a performance obligation but believe this area requires further clarification." (Apple) ${ }^{456}$

„The solution of allocating the transaction price to separate performance obligations contemplated by the board is quite understandable from our point of view. In our opinion it leads to sensible outcomes. Nevertheless, we consider the allocation of the transaction price to separate performance obligations to be only necessary for a few industrial sectors." (Volkswagen) ${ }^{457}$

Die Überzeugungskraft beruhte insofern auf dem Ausdruck von Anerkennung, was die Auseinandersetzung der Unternehmen mit den Regelungsvorschlägen demonstriert.

Bei den Standardsetzern stellte die Vermittlung von Respekt gegenüber den Unternehmen das häufigste Mittel zur Erzeugung von Glaubwürdigkeit dar. Dies lässt sich darauf zurückführen, dass auf diese Weise der Einbezug der Adressaten in den Standardsetzungsprozess verdeutlicht werden konnte. ${ }^{458}$

„This discussion paper invites comments on the boards' preliminary views on a single, contract-based revenue recognition model.“(IASB/FASB) ${ }^{459}$

„In response to questions from respondents, the boards decided to specify that a residual approach might be a suitable technique for estimating the stand-alone selling price of a good or service." (IASB/FASB) $)^{460}$

${ }^{454}$ Vgl. auch Stenka (2014), S. 21.

455 Vgl. zur Legitimität durch Einschmeicheln Allen/Caillouet (1994), S. 48.

${ }^{456}$ Apple (2009), S. 2.

457 Volkswagen (2012), S. 5.

458 Vgl. auch Stenka (2014), S. 18.

${ }^{459}$ DP (2008), S3.

460 IFRS 15.BC270. 
Glaubwürdigkeit wurde zudem durch die Vermittlung von Autorität erzeugt, die auf eigener und fremder Geltungswirkung beruhte. Während die Unternehmen im Standardsetzungsprozess am häufigsten auf die ökonomische Realität sowie besonders im Exposure Draft auch auf andere Institutionen und damit auf fremde Autorität Bezug nahmen, verwiesen die Standardsetzer am häufigsten auf den eigenen Regelungsvorschlag und folglich auf die eigene Autorität. ${ }^{461}$ Die ökonomische Realität eignete sich für Unternehmen als äußerer Maßstab, der insofern eine objektive Überzeugungskraft aufweist.
„As the standard is currently drafted, we believe we may be precluded from separa- ting software license and PCS obligations in bundled transactions since the resources, costs and profit margin would not be separately identifiable. This would result in financial statements that are not reflective of the economics of the transaction."

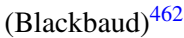
„We believe application of the model proposed by the Revised ASU will result in accounting for items as performance obligations when they are no more than sales incentives, which will result in accounting for these items in a manner that is different from the way in which the underlying business is managed." (General Motors) ${ }^{463}$

Auch durch den Verweis von Unternehmen auf andere Institutionen, wie etwa Bürgschaftsbanken oder andere Standardsetzungskomitees, wurde auf eine fremde Autorität verwiesen, die als objektiver Bezugspunkt den Äußerungen Glaubwürdigkeit verlieh.

„There are significant concerns in the surety community about any approach that diminishes consistency and increases subjectivity." (DPR Construction) ${ }^{464}$

„We agree with EFRAG that only the entity's own customary business practice should be considered when determining distinct goods and services, rather than the business practice of any other entity." (Maersk) ${ }^{465}$

Die Standardsetzer nahmen indes häufig auf eigene Vorschläge Bezug, wodurch die Autorität auf die eigene Standardsetzungskompetenz gestützt wurde. Auch vorgenommene Änderungen konnten hierdurch aufgezeigt werden, was wiederum den Einbezug der Adressaten bei gleichzeitigem Aufzeigen des Fortschritts der Regelungsentwicklung verdeutlichte.

\footnotetext{
461 Vgl. Appendix, VIII und X.

462 Blackbaud (2010), S. 4.

463 General Motors (2012), S. 5.

${ }^{464}$ DPR Construction (2010), S. 1.

${ }^{465}$ Maersk (2010), S. 2.
} 
„Compared with the proposals in the discussion paper, there would be fewer instances under the proposed requirements in which the transaction price would be allocated using estimates of stand-alone selling prices." (IASB/FASB) ${ }^{466}$

„Hence, the boards affirmed their proposal in the 2010 exposure draft not to specify a hierarchy. Instead, the boards decided that it was important to emphasise that an entity should maximise the use of observable inputs when developing estimates of standalone selling prices" (IASB/FASB) $)^{467}$

Im Hinblick auf die rhetorische Strategie von Unternehmen legt die während des gesamten Standardsetzungsprozesses häufigere Verwendung von Ethos gegenüber Logos in Verbindung mit der hohen Verwendung von Expertise sowie der Anknüpfung an die ökonomische Realität folglich nahe, dass Unternehmen sich durch fachspezifische, auf die wirtschaftliche Realität bezugnehmende Kommentierungen der Bilanzierungsalternativen als standardsetzungskompetent positionieren, um hierdurch Glaubwürdigkeit zu erlangen und die Standardsetzer zu überzeugen. In Bezug auf die rhetorische Strategie der Standardsetzer zeigt die während des Standardsetzungsprozesses tendenziell häufigere Verwendung von Logos in Verbindung mit der im Rahmen von Ethos häufigen Verwendung von Respekt sowie der Betonung der eigenen Standardsetzungskompetenz, dass die Standardsetzer Entscheidungen auf Basis rationaler, ihre Zuständigkeit betonenden Begründungen, indes unter Berücksichtigung bzw. Adressierung der Stellungnahmen rechtfertigen, um hierdurch die Akzeptanz der Regelungsvorschriften sicherzustellen und dem Legitimationserfordernis Rechnung zu tragen.

\subsection{Verwendung rhetorischer Stilmittel bei allen Überzeugungsarten}

Als rhetorische Stilmittel wurden von den Unternehmen bei den untersuchten Bilanzierungsalternativen am häufigsten die User-Metonymie sowie die Antithese verwendet (Abbildung 3.10) ${ }^{468}$. Metaphern und Übertreibungen wurden weniger als halb so häufig genutzt; die Verwendung von Wiederholungen und rhetorischen Fragen erfolgte verhältnismäßig selten. Der metonymische Verweis in Form von Most/Some wurde nicht verwendet, was jedoch in der standardsetzerspezifischen Anknüpfung des Stilmittels begründet liegt.

\footnotetext{
${ }^{466}$ ED BC (2010), BC117.

${ }^{467}$ Re-ED BC (2011), BC185.

468 Vgl. Appendix, IX.
} 


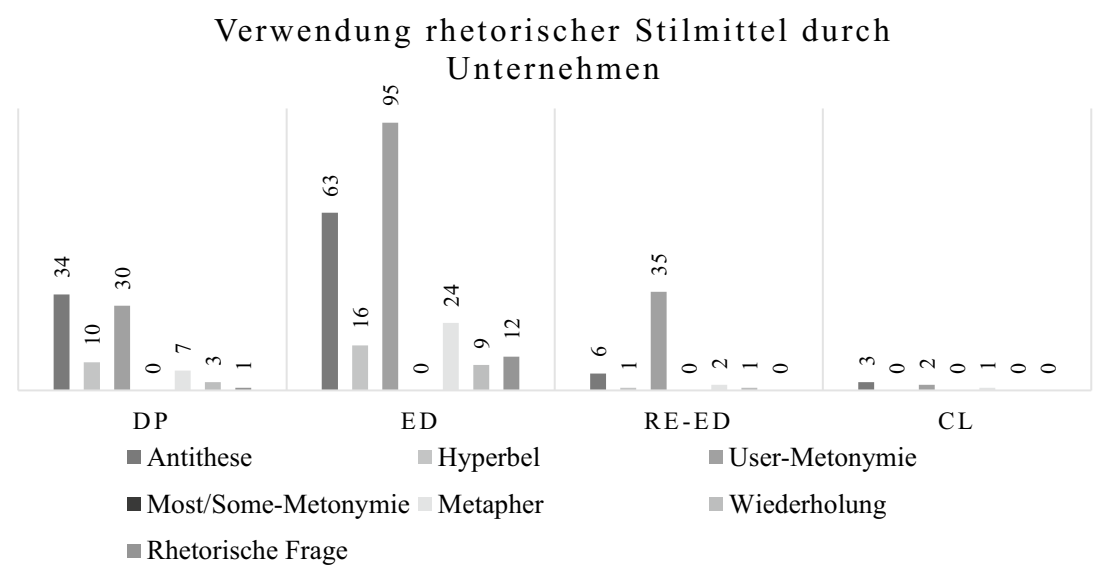

Abbildung 3.10 Codier-Häufigkeit der von Unternehmen verwendeten rhetorischen Stilmittel in der jeweiligen Kommentierungsphase

Im Gegensatz hierzu stellte die Most/Some-Metonymie das von den Standardsetzern am häufigsten genutzte rhetorische Stilmittel dar (Abbildung 3.11) ${ }^{469}$. Diese sprachliche Wahl diente zum Aufzeigen des Einbezugs der am Standardsetzungsprozess Beteiligten und wurde ausschließlich in Zusammenhang mit der Vermittlung von Respekt, mithin zur Überzeugung durch Ethos, verwendet. $^{470}$ So wurde mit dem Verweis auf „most respondents“ einerseits die Umsetzung einer bestimmten Bilanzierungsalternative durch Marginalisierung gegensätzlicher Regelungsvorschläge mit dem Verweis auf ,,some respondents“ als mehrheitsfähige Lösung dargestellt. ${ }^{471}$

„Most respondents agreed with the boards' proposals in the discussion paper, although some suggested that the boards should consider whether: [...] to constrain the use of estimates and specify a hierarchy for the basis of allocation [...].

[...] The boards reaffirmed the view they expressed in the discussion paper that they will not preclude or prescribe any particular method for estimating a stand-alone selling price [...].“ (IASB/FASB $)^{472}$

${ }^{469}$ Vgl. Appendix, XI.

${ }^{470}$ Die Kookkurrenz von Most/Some und Respekt zeigt 42 von 42 Codierungen, mithin $100 \%$.

471 Vgl. auch Stenka (2014), S. 18.

${ }^{472}$ ED BC (2010), BC114, BC120. 
Andererseits wurden durch den Verweis auf „some respondents“ jedoch auch Zugeständnisse als Kompromisslösung verdeutlicht.

„The practical expedient is intended to address the concerns raised by some respondents that they frequently would have to identify numerous performance obligations and account for them separately." (IASB/FASB) $)^{473}$

Das rhetorische Stilmittel der Antithese wurde von den Standardsetzern am häufigsten im Discussion Paper eingesetzt; die Verwendung der User-Metonymie erfolgte während des Standardsetzungsprozesses indes in etwa konstant. Metaphern, Wiederholungen und rhetorische Fragen wurden von den Standardsetzern nicht verwendet, was auf die mit der Vermittlung von Pathos in Zusammenhang stehende Anknüpfung dieser Stilmittel zurückgeführt werden kann.

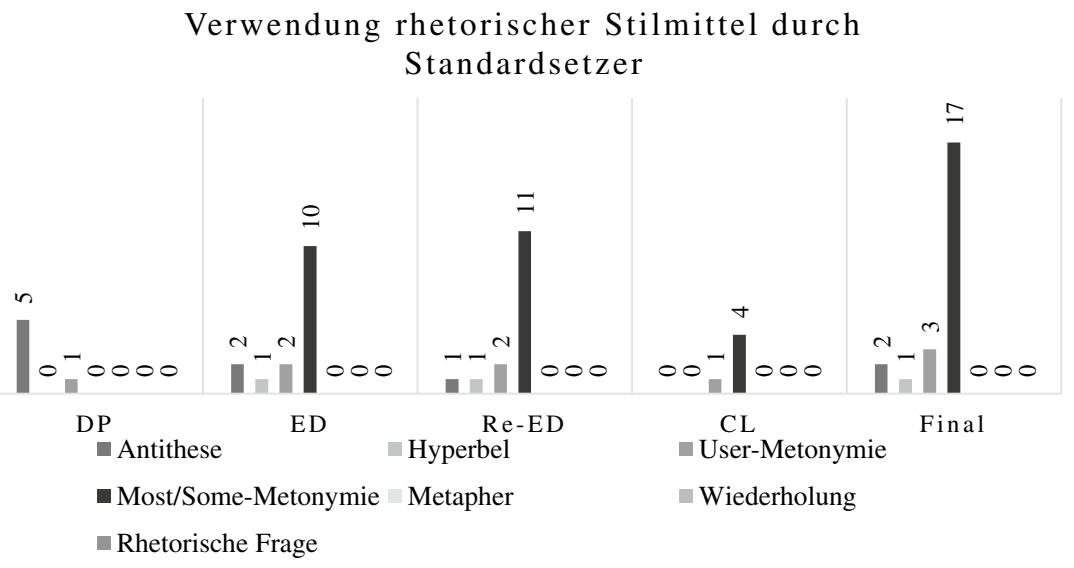

Abbildung 3.11 Codier-Häufigkeit der von den Standardsetzern verwendeten rhetorischen Stilmittel in der jeweiligen Standardsetzungsphase

${ }^{473}$ Re-ED BC (2011), BC81. 
Sowohl Unternehmen als auch Standardsetzer nutzten die User-Metonymie überwiegend in Zusammenhang mit konzeptionellen Gründen, mithin zur Überzeugung durch Logos, ${ }^{474}$ was insoweit in Einklang mit der Zwecksetzung und Wortwahl des Rahmenkonzepts steht.

„The company proposes that the definition of a performance obligation [...] be amended to include the concept that the performance obligation must have stand-alone customer value as a good or service. Otherwise, the company is concerned that performance obligations may be disaggregated to a level of detail which would be unnecessary and not useful to financial statement users." (IBM) ${ }^{475}$

„When the goal is to improve overall contract profitability, reporting separate gain or loss on interdependent obligations within the contract is misleading to financial statement users who will only see a portion of the results of decisions that have been made." (Kiewit) ${ }^{476}$

„The boards were concerned that requiring an entity to account separately (and estimate a stand-alone selling price) for a good or service that is not capable of providing a benefit to the customer might result in information that would not be relevant to users of financial statements." (IASB/FASB $)^{477}$

Der User-Verweis fungiert insofern als zusätzliche Rechtfertigung einer Bilanzierungslösung, der als Metonymie keine Konkretisierung der Adressaten erfordert. 478

Die Unternehmen verwendeten zudem Antithesen in Zusammenhang mit der Vermittlung von Respekt, mithin zur Überzeugung durch Ethos, ${ }^{479}$ um ein gewisses $\mathrm{Ma} \beta$ an Zustimmung und Anerkennung zu zeigen, was die darauffolgende Ablehnung bzw. Kritik glaubwürdiger erscheinen ließ.480

„We generally agree that the transaction price should be allocated to all separate performance obligations in a contract in proportion to the stand-alone selling price of the

\footnotetext{
474 Die Kookkurrenz von User und den Subkategorien der konzeptionellen Gründe zeigt für Unternehmen 108 von 162 Codierungen und für die Standardsetzer 6 von 9 Codierungen, mithin jeweils $66,67 \%$.

475 IBM (2009), S. 3.

476 Kiewit (2010), S. 4.

${ }^{477}$ IFRS 15.BC97.

478 Vgl. auch Stenka (2014), S. 19; Young (2003), S. 629.

${ }^{479}$ Die Kookkurrenz von Antithese und Respekt zeigt 104 von 106 Codierungen, mithin $98,11 \%$.

${ }^{480}$ Vgl. auch Stenka (2014), S. 21.
} 
good or service underlying each of those performance obligations. However, we strongly recommend maintaining the FASB's provision under ASC 605-25-30.“ (Deutsche Telekom $)^{481}$

Die Standardsetzer verwendeten ebenfalls Antithesen, um Respekt gegenüber den Unternehmen sowie der Anwendbarkeit der Regelungen $\mathrm{zu}$ verdeutlichen und hierdurch die Akzeptanz für die Regelungsvorschläge sicherzustellen. ${ }^{482}$

\begin{abstract}
„Because those goods and services are assets, the computer manufacturer's promise to deliver a computer could, in concept, be separated into many performance obligations. In practice, however, identifying many performance obligations in a contract such as the above example and accounting for them separately would be unnecessarily complex." (IASB/FASB) $)^{483}$
\end{abstract}

Die Verwendung rhetorischer Stilmittel verdeutlicht ebenso wie der Einsatz der einzelnen Überzeugungsarten den Einfluss sprachlich-rhetorischer Aspekte auf den Standardsetzungsprozess. Zentral für die Beurteilung der diskursiven Auseinandersetzung ist demzufolge das ,Verständnis, dass Sprache integraler Bestandteil des gesellschaftlichen Lebens und grundlegend für politische Verhandlungen auf verschiedenen Ebenen ist."484 In einem letzten Schritt werden daher die zwei dominanten story-lines erläutert, die unter Anwendung der argumentativen Diskursanalyse ${ }^{485}$ bei den untersuchten Bilanzierungsalternativen im Standardsetzungsprozess von IFRS 15 identifiziert wurden.

\title{
3.2.3 Rekonstruktion relevanter story-lines im Sinne der argumentativen Diskursanalyse
}

\subsubsection{Rekonstruktion der "user needs" story-line}

Sowohl die Unternehmensstellungnahmen als auch die Standardsetzungsdokumente weisen in allen Standardsetzungsphasen zwei zentrale story-lines auf, mithilfe derer Regelungsänderungen erklärt werden. Hierbei handelt es sich um Darstellungen der ,user needs“ und der ,economic reality“, die durch ihre narrative Verwendung die Standardsetzung strukturieren. Beide story-lines sind

\footnotetext{
${ }^{481}$ Deutsche Telekom (2010), S. 12.

$482 \mathrm{Vgl}$. auch Masocha/Weetman (2007), S. $86 \mathrm{f}$.

${ }^{483}$ DP (2008), $3.21 \mathrm{f}$.

${ }^{484}$ Stenka (2014), S. 13 (Übersetzung der Verfasserin).

485 Vgl. zur argumentativen Diskursanalyse Hajer (1997), S. 110-118.
} 
dabei Ausdruck diskursiven Erfolgs, d. h. der Etablierung eines bestimmten Vokabulars sowie der Deutung des etablierten Vokabulars ${ }^{486}$. Als solche verdeutlichen sie die Anschlussfähigkeit ebenso wie die Marginalisierung bestimmter Bedeutungsgehalte. ${ }^{487}$ Sie stellen die diskursiven Strukturen des Standardsetzungsprozesses und folglich den Rahmen, innerhalb derer ein Wandel von Rechnungslegungsvorschriften stattfindet, ${ }^{488}$ dar.

Die story-line der Nutzerbedürfnisse gründet auf dem Zweck der Entscheidungsnützlichkeit und findet die prägnanteste Ausprägung in der metonymischen User-Verwendung. Die Bedürfnisse und Interessen der Nutzer werden dabei sowohl von den Unternehmen als auch von den Standardsetzern ,through the prism of the conceptual framework" interpretiert und als Rechtfertigung für die Angemessenheit eines Regelungsvorschlags konstituiert. ${ }^{489}$ Während Unternehmen sich durch die Darstellung der Nutzerbedürfnisse von einer eigennutzorientierten Kommentierung distanzieren bzw. mit der Nutzergruppe sympathisieren ${ }^{490}$ und damit Kongruenz gegenüber den von den Standardsetzern normierten Rahmenkonzeptanforderungen signalisieren, ${ }^{491}$ erfüllen die Standardsetzer durch die Narration der Nutzerbedürfnisse das Legitimationserfordernis, indem sie als Sprecher für diese Gruppe eintreten und Entscheidungen in ihrem Namen rechtfertigen. ${ }^{492}$ Hierdurch wird ein Bild der Nutzer konstruiert, das als selbstverständliche und unzweifelhafte Referenz bei der Ausgestaltung von Rechnungslegungsvorschriften dient. ${ }^{493}$

Auch im Standardsetzungsprozess von IFRS 15 hat sich die story-line der Nutzerbedürfnisse etabliert, wobei sie nicht bei allen Bilanzierungsalternativen die gleiche Anschlussfähigkeit besaß. So stellten die Unternehmen bereits im Discussion Paper die Nutzerbedürfnisse bei den Kriterien zur Aufteilung der Leistungsverpflichtungen in den Vordergrund und rechtfertigten auf diese Weise die Notwendigkeit eines differenzierten Aufteilungskriteriums. Die gleiche story-line diente auch den Standardsetzern zur Rechtfertigung des überarbeiteten Regelungsvorschlags.

\footnotetext{
486 Vgl. Baumann (2006), S. $135 \mathrm{f}$.

487 Vgl. Brand (2012), S. 218.

488 Vgl. Young (1994), S. 103.

${ }^{489}$ Vgl. Young (2006), S. 593-595 (auch Zitat, S. 594); Williams/Ravenscroft (2015), S. 765; Stenka/Jaworska (2019), S. 3.

${ }^{490}$ Vgl. Weetman u. a. (1996), S. 72, 75.

491 Vgl. Stenka/Jaworska (2019), S. 9.

492 Vgl. Young (2003), S. 629; Young (2006), S. 593.

493 Vgl. Stenka/Jaworska (2019), S. 3.
} 
„Current contract accounting standards require an estimate to be made of a single gross margin for all production units expected to be delivered. [...] A consistent profit margin estimate for all goods delivered under the contract provides financial statement users with more decision-useful contract information than separating performance obligations and potentially recognizing different profit margins on the same product under the same contract with a single customer." (United Technologies) ${ }^{494}$

„Even if a good or service has a distinct function, the boards decided that it should be accounted for as separate performance obligation only if it also has a distinct profit margin. If a good or service does not have a distinct profit margin, the boards were concerned that requiring an entity to estimate a selling price for that good or service might result in information that would not be useful to users of financial statements." $(\text { IASB/FASB })^{495}$

Die gleiche story-line wurde wiederum auch von den Unternehmen bei der Kommentierung des Exposure Drafts sowie von den Standardsetzern zur Rechtfertigung im Re-Exposure Draft verwendet.

\begin{abstract}
„Our concern with applying a more theoretical view of what could potentially be sold separately will be difficult to apply in practice and will not result in more information for users of the financial statement." (Cisco) $)^{496}$

„In the 2010 exposure draft, the boards proposed that a good or service is distinct if it is sold separately (by the entity or by another entity) or if it could be sold separately. The boards were concerned that requiring an entity to account separately (and estimate a stand-alone selling price) for a good or service that is not capable of being sold separately might result in information that would not be useful to users of financial statements." (IASB/FASB) ${ }^{497}$
\end{abstract}

Hierbei wird deutlich, dass Unternehmen und Standardsetzer ,einen passiven homogenen aber gleichzeitig abstrakten Nutzer konstruieren, es sich insoweit eher um ein vages rhetorisches Konstrukt als um einen greifbaren realen Stakeholder handelt." “98

Der Standardsetzungsprozess von IFRS 15 zeigt hingegen auch die Grenzen der Anschlussfähigkeit dieser story-line bzw. die Dominanz der ,economic substance" story-line. So wurden die Nutzerbedürfnisse im Discussion Paper und ReExposure Draft am häufigsten von Unternehmen der Telekommunikationsbranche

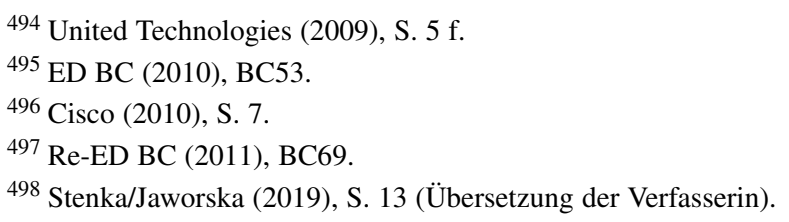


in Zusammenhang mit den Reglungsvorschlägen zur Aufteilung des Transaktionspreises dargestellt, ${ }^{499}$ um die Notwendigkeit einer Regelungsänderung in Frage zu stellen.

„Current revenue recognition principles under US GAAP, and as generally applied by the telecommunications industry, ensure that revenue is not recognised for payments from customers that are contingent on the delivery of future services. We believe that this is of great value to users of the financial statements as recognised revenue is closely related to billings and cash received from the customer and the impact of management estimation is reduced. It is our belief that discarding the contingent revenue cap would be viewed negatively by users of the financial statements." (Vodafone $^{500}$

„Our primary concern with the proposed standard has been, and continues to be, the reduction in the usefulness of information to the users of our financial statements should this proposed standard become effective." (Sprint Nextel) ${ }^{501}$

Obwohl Feldstudien und Umfragen mit Nutzern die Darstellung der Nutzerbedürfnisse bestätigten und auch das Staff eine Ausweitung der Residualwertmethode unter Verwendung der user needs story-line empfahl, ${ }^{502}$ lehnten die Standardsetzer die Umsetzung einer im Ergebnis zu den vorherigen Regelungsvorschriften ähnlichen Bilanzierungslösung ab. Anstelle der story-line der Nutzerbedürfnisse begründeten die Standardsetzer ihre Entscheidung indes anhand der ,underlying economics“ ${ }^{\text {503 }}$, die die zweite relevante story-line darstellt.

\subsubsection{Rekonstruktion der "economic reality" story-line}

Die story-line der ökonomischen Realität basiert auf dem Anspruch der Rechnungslegung, die zugrundeliegende wirtschaftliche Substanz einer Transaktion abzubilden, und findet ihre Ausprägung in dem Realitätsbezug sowie den Verweisen auf die glaubwürdige Darstellung. Die wirtschaftliche Substanz einer Transaktion verlangt insofern an eine Realitätsabbildung, die eine ,correspondence between accounting and an object out there aufzeigt. ${ }^{504}$ Die ökonomische

\footnotetext{
${ }^{499}$ Im Discussion Paper stammen 18 von 30 (60,00 \%) und im Re-Exposure Draft 27 von 35 $(77,14 \%)$ Codierungen von Unternehmen der Telekommunikationsbranche.

500 Vodafone (2009), S. 13.

501 Sprint Nextel (2012), S. 1.

${ }^{502}$ Vgl. Technical Staff (2011b), Agenda Paper 4B/147B, Rz. 38 (b).

503 IFRS 15.BC292.

${ }^{504}$ Vgl. Young (2003), S. 628 (auch Zitat, Hervorhebungen im Original).
} 
Realität ist jedoch von der eingenommenen Perspektive abhängig, ${ }^{505}$ weshalb es sich anstelle einer von den Rechnungslegungsvorschriften und der Rechnungslegungspraxis unabhängigen und folglich objektiv existierenden Realität ${ }^{506}$ vielmehr um eine Darstellung dieser bzw. um ein von dieser konstruiertes Abbild ${ }^{507}$ handelt. Durch „,commonsense reasoning“ wird diese Darstellung jedoch gerade als objektive Realität impliziert; ${ }^{508}$ von Unternehmen als die der wirtschaftlichen Substanz entsprechende Bilanzierung der Transaktionen und von den Standardsetzern als die dem Rahmenkonzept entsprechende glaubwürdige Darstellung der wirtschaftlichen Substanz der Transaktionen. ${ }^{509}$ Hierdurch wird ein Bild der ökonomischen Realität konstruiert, das im Standardsetzungsprozess - ebenso wie die Nutzerbedürfnisse - als rhetorisches Konstrukt bei der Regelungsausgestaltung dient.

Im Standardsetzungsprozess von IFRS 15 wurde die story-line der ökonomischen Realität sowohl von den Unternehmen als auch von den Standardsetzern verwendet, wobei die Bedeutung diskursiver Strukturen deutlich wird. So befürworteten Unternehmen der Softwarebranche im Discussion Paper etwa die Aufteilung des Transaktionspreises auf Basis der relativen Einzelveräußerungspreise aufgrund der hierdurch verbesserten Abbildung der wirtschaftlichen Realität.

\begin{abstract}
„We believe that the proposed model will improve upon existing revenue recognition guidance by allowing the allocation of the transaction price to the performance obligations on the basis of the entity's standalone selling prices of the goods or services underlying those performance obligations. [...] The change in the measurement approach from current practice to the proposed standard will allow companies to better reflect the underlying economics of an arrangement." (Intel) ${ }^{510}$
\end{abstract}

Unternehmen der Telekommunikationsbranche lehnten dieselbe Regelungsvorschrift hingegen unter Verwendung der gleichen story-line ab.

„In multiple element transactions, the DP proposes the allocation, at the inception of the contract, of the transaction price in proportion to the stand-alone selling price of each performance obligation (the relative stand-alone selling price method). This method does not reflect the substance of the telecommunications business where the

\footnotetext{
505 Vgl. Wüstemann/Wüstemann (2011), S. 12.

${ }^{506}$ Vgl. Lee (2006b), S. 2 f.; Alexander/Archer (2003), S. 5 f.

${ }^{507}$ Vgl. Loux (2006), S. 7 f.

${ }^{508}$ Vgl. Hines (1991), S. 315-322 (auch Zitat, S. 318).

509 Vgl. Wüstemann/Wüstemann (2011), S. 13.

${ }^{510}$ Intel (2009), S. 16.
} 
huge amount of capital expenditures for network is earned from the rendering of services rather than through the sale of equipments to customers." (Telecom Italia) ${ }^{511}$

Die Standardsetzer teilten indes das Verständnis der Softwarebranche und hielten an dem im Vergleich zur vorherigen Rechnungslegungspraxis geänderten Regelungsvorschlag fest.

„The boards proposed that an entity should allocate the transaction price in proportion to the stand-alone selling prices of the promised goods or services. They noted that an allocation based on stand-alone selling prices faithfully depicts the different margins that may apply to promised goods or services." (IASB/FASB) $)^{512}$

Die Telekommunikationsunternehmen lehnten die Aufteilung auf Basis relativer Einzelveräußerungspreise auch im Exposure Draft und Re-Exposure Draft aufgrund der von der ökonomischen Realität abweichenden Bilanzierung ab.

„For bundled arrangements including performance obligations that are fulfilled at different moments in time, revenue should only be allocated to those obligations to the extent that the allocated revenue does not exceed the legally enforceable payments due from the customer under the terms of the contract without the delivery of future services. We consider that applying the contingent revenue cap would result in financial information that better reflects the economics of this type of transactions." (Telefónica) $^{513}$

„However, we believe that the proposed standard results in accounting which is even more disconnected from the underlying economics of our contracts and does not appropriately consider the value exchange from the customer' s perspective." (Sprint Nextel) $)^{514}$

Im Gegensatz hierzu bestätigten die Standardsetzer die Regelungsänderung gleichsam die Nicht-Umsetzung einer Contingent-Revenue-Cap-Methode damit, dass die Regelungen der Bilanzierung der zugrundeliegenden wirtschaftlichen Substanz - insbesondere im Hinblick auf den in der Praxis eigenständigen Nutzen von Mobilfunktelefonen - ${ }^{515}$ eher gerecht werden.

\footnotetext{
511 Telecom Italia (2009), S. 3.

512 ED BC (2010), BC113.

513 Telefónica (2010), S. 7.

514 Sprint Nextel (2012), S. 4.

515 Vgl. Teixeira (2014), S. 7.
} 
„Additionally, the boards decided not to introduce an exception to the revenue model for telecommunications and similar contracts because they do not view those contracts to be unique. Furthermore, the boards decided that the proposed requirements would provide a more consistent basis for recognising revenue and would produce results in accounting that more closely match the underlying economics of transactions." $(\mathrm{IASB} / \mathrm{FASB})^{516}$

Dies verdeutlicht einerseits die Dominanz der story-line der ökonomischen Realität gegenüber der story-line der Nutzerbedürfnisse bei der Ausgestaltung der Regelungen zur Aufteilung des Transaktionspreises. Andererseits werden die diskursiven Grenzen des Standardsetzungsprozesses deutlich. So entspricht die unternehmens- bzw. branchenspezifische Deutung der ökonomischen Realität der Telekommunikationsunternehmen eher einer subjektiven anstatt der als objektiv konstruierten Realität, was die fehlende Überzeugungskraft dieser Unternehmenskommentierungen erklärt. Der Wandel von Rechnungslegungsvorschriften verläuft daher nicht nur in den Grenzen der Standardanwendung, sondern wird auch von den diskursiven Strukturen determiniert. Sowohl Unternehmen als auch Standardsetzer sollten sich dieser aufgrund ihres wesentlichen Einflusses auf das Ausmaß der Überarbeitung bestehender sowie der Entwicklung neuer Regelungsvorschriften bei der Standardsetzung bewusst sein.

Open Access Dieses Kapitel wird unter der Creative Commons Namensnennung 4.0 International Lizenz (http://creativecommons.org/licenses/by/4.0/deed.de) veröffentlicht, welche die Nutzung, Vervielfältigung, Bearbeitung, Verbreitung und Wiedergabe in jeglichem Medium und Format erlaubt, sofern Sie den/die ursprünglichen Autor(en) und die Quelle ordnungsgemäß nennen, einen Link zur Creative Commons Lizenz beifügen und angeben, ob Änderungen vorgenommen wurden.

Die in diesem Kapitel enthaltenen Bilder und sonstiges Drittmaterial unterliegen ebenfalls der genannten Creative Commons Lizenz, sofern sich aus der Abbildungslegende nichts anderes ergibt. Sofern das betreffende Material nicht unter der genannten Creative Commons Lizenz steht und die betreffende Handlung nicht nach gesetzlichen Vorschriften erlaubt ist, ist für die oben aufgeführten Weiterverwendungen des Materials die Einwilligung des jeweiligen Rechteinhabers einzuholen.

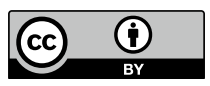

${ }^{516}$ Re-ED BC (2011), BC197; IFRS 15.BC292. 